Aus der Abteilung Psychosomatische Medizin und Psychotherapie

(Prof. Dr. med. C. Herrmann-Lingen)

im Zentrum Psychosoziale Medizin

der Medizinischen Fakultät der Universität Göttingen

\title{
Beziehungen erhöhten arteriellen Blutdrucks zu Lebensqualität, Problembelastung und Risikoverhalten im Jugendalter
}

\author{
INAUGURAL - DISSERTATION \\ zur Erlangung des Doktorgrades \\ der Medizinischen Fakultät \\ der Georg-August-Universität zu Göttingen \\ vorgelegt von \\ Angela Stefanie Berendes \\ aus \\ Bamberg
}


D e k a n:

I. Berichterstatter:

II. Berichterstatter/in:

Tag der mündlichen Prüfung:
Prof. Dr. rer. nat. H. K. Kroemer

Prof. Dr. med. C. Herrmann-Lingen

Priv.-Doz. Dr. med. R. Wachter

30. Oktober 2013 


\section{Inhaltsverzeichnis}

Abkürzungsverzeichnis

1.1 Wachsende klinische Bedeutung der essentiellen arteriellen Hypertonie

1.2 Essentielle arterielle Hypertonie - bereits bei Kindern und Jugendlichen ein zunehmendes Problem

1.3 Existiert ein Zusammenhang zwischen arterieller Hypertonie und Lebensqualität?

1.4 Zielsetzung dieser Arbeit

1.5 Hypothesen zum erhöhten arteriellen Blutdruck im Jugendalter

1.5.1 Hypothesen zur Prävalenz

1.5.2 Hypothesen bezüglich kardiovaskulärer Risikofaktoren

1.5.3 Hypothesen bezüglich psychosomatischer Korrelate

2.1 Beschreibung der KiGGS-Studie

2.2 Blutdruckmessung im Kinder- und Jugendgesundheitssurvey

2.3 Bestimmung der Lebensqualität

2.4 Bestimmung der psychosozialen Probleme und Stärken

2.5 Bestimmung der Schutzfaktoren

2.5.1 Personale Ressourcen

2.5.2 Soziale Ressourcen

2.5.3 Familiäre Ressourcen 
2.7.1 Allgemeine Angaben 25

2.7.2 Definition der Blutdruckvariablen 26

$\begin{array}{lll}2.7 .3 & \text { Beschreibung des Kollektivs } & 28\end{array}$

2.7.4 Überprüfung der Korrelation 29

2.7.5 Überprüfung der Verteilung von Lebensqualität, psychosozialen Problemen und Stärken sowie Schutzfaktoren bei normotensiven und hypertensiven Jugendlichen

2.7.6 Explorative Testung der normotensiven und hypertensiven Jugendlichen auf weitere Unterschiede

2.7.7 Multivariate Testung im Modell $1 \mathrm{~b}$

2.7.8 Lebensqualität und psychosoziale Probleme als abhängige Variablen

\section{Ergebnisse}

3.1 Beschreibung des Kollektivs und der Häufigkeitsverteilung eines erhöhten arteriellen Blutdrucks

3.2 Korrelation zwischen Subskalen und übergreifendem Messwert sowie zwischen Eigen- und Elternangaben bei Lebensqualität und psychosozialen Problemen

3.3 Überprüfung der Verteilung von Lebensqualität, psychosozialen Problemen und Schutzfaktoren bei normotensiven und hypertensiven Jugendlichen

3.4 Überprüfung von Lebensqualität sowie von psychosozialen Problemen und Stärken im Modell mit möglichen Confoundern

3.4.1 Univariate Überprüfung der relevanten Variablen

3.4.2 Multivariate Testung im Modell 1

3.5 Explorative Überprüfung der normotensiven und hypertensiven Jugendlichen auf weitere Unterschiede 
3.5.1 Chi-Quadrat-Test zur explorativen Überprüfung der beiden Gruppen auf weitere Unterschiede

3.5.2 Multivariate Überprüfung der Confounder im Modell 1

3.6 Multivariate Testung der relevanten Variablen im Modell 1b

3.6.1 Lebensqualität und psychosoziale Probleme im multivariaten Modell $1 b$

3.7 Güte der Modellanpassung und in die Analyse einbezogene Fälle

3.8 Lebensqualität und psychsoziale Probleme als abhängige Variablen im multivariaten Modell

4.1 Zentrale Erkenntnisse der vorliegenden Untersuchung

4.2 Bedeutendste Ergebnisse im Vergleich zu anderen Studien

4.2.1 Steigende Prävalenz des erhöhten arteriellen Blutdrucks im Jugendalter

4.2.2 Assoziation besserer Lebensqualität und psychischer Gesundheit mit erhöhtem arteriellen Blutdruck

4.2.3 Zum Widerspruch zwischen ungünstigem Freizeitverhalten und dennoch besserer sozialer Angepasstheit

4.2.4 Hypothese der „learned hypertension“

4.2.5 Affektrepression als mögliche Teilursache der Blutdruckerhöhung

4.3 Bedeutung für den klinischen Alltag

4.4 Stärken und Schwächen der Arbeit

5. Zusammenfassung 


\section{Abkürzungsverzeichnis}

\begin{tabular}{|c|c|}
\hline Abkürzung & Bedeutung \\
\hline Abb. & Abbildung \\
\hline ADHS & Aufmerksamkeitsdefizit-Hyperaktivitätssyndrom \\
\hline $\mathrm{BD}$ & Blutdruck \\
\hline $\mathrm{BMI}$ & Body Mass Index \\
\hline Bsp. & Beispiel \\
\hline bzw. & beziehungsweise \\
\hline ca. & circa \\
\hline $\mathrm{cm}$ & Zentimeter \\
\hline $\mathrm{CSHCN}$ & Children with Special Health Care Needs \\
\hline d.h. & das heißt \\
\hline DSM-IV & $\begin{array}{l}\text { Diagnostic and Statistical Manual of Mental Disorders (Diagnos- } \\
\text { tisches und statistisches Handbuch psychischer Störungen) }\end{array}$ \\
\hline EEG & Elektroenzephalografie \\
\hline et al. & et alii (und andere) \\
\hline $\mathrm{HF}$ & Herzfrequenz \\
\hline ICD-10 & $\begin{array}{l}\text { International Statistical Classification of Diseases and Related } \\
\text { Health Problems (Internationale statistische Klassifikation der } \\
\text { Krankheiten und verwandter Gesundheitsprobleme) }\end{array}$ \\
\hline $\mathrm{kg}$ & Kilogramm \\
\hline KiGGS & Kinder- und Jugendgesundheitssurvey \\
\hline KINDL-R & Kinder-Lebensqualitätsfragebogen \\
\hline$-2 \mathrm{LL}$ & negative doppelte Wert des Logarithmus \\
\hline $\mathrm{m}^{2}$ & Quadratmeter \\
\hline mind. & mindestens \\
\hline $\min ^{-1}$ & pro Minute \\
\hline $\mathrm{mmHg}$ & Millimeter-Quecksilbersäule \\
\hline
\end{tabular}


MTA

$\mathrm{N}$

NHANES

NHBPEP

OR

Perz.

SDQ

sign.

S.O.

Std.

Tab.

u.a.

$\mathrm{vgl}$.

vs.

ZUMA
Medizinisch technische Assistentin

Anzahl

National Health and Nutrition Examination Survey

National High Blood Pressure Education Programm

Odds Ratio

Perzentil

Strength and Difficulties Questionnaire

signifikant

siehe oben

Stunde

Tabelle

unter anderem

vergleiche

gegenüber

Zentrum für Umfragen, Methoden und Analysen 


\section{Abbildungs- und Tabellenverzeichnis}

\section{Abbildungen}

Abb.1 Häufigkeitsverteilung des erhöhten arteriellen Blutdrucks in Abhängigkeit S.33 von Alter und Geschlecht

Abb.2 Häufigkeitsverteilung des erhöhten systolischen Blutdrucks in Abhängig- S.34 keit von Alter und Geschlecht

Abb.3 Häufigkeitsverteilung des erhöhten diastolischen Blutdrucks in Abhängig- S.35 keit von Alter und Geschlecht

Abb.4 Überblick über die Unterschiede zwischen hypertensiven und normotensiven Jugendlichen in den einzelnen Bereichen der Lebensqualität anhand der Eigenangaben

Abb.5 Überblick über die Unterschiede zwischen normotensiven und hyperten- $\quad \mathrm{S} .42$ siven Jugendlichen in den Bereichen der psychosozialen Schwierigkeiten und Stärken anhand der Eigenangaben

Abb.6 Überblick über die Unterschiede zwischen hypertensiven und normotensiven Jugendlichen in den einzelnen Bereichen der Lebensqualität anhand der Elternangaben

Abb.7 Überblick über die Unterschiede zwischen normotensiven und hypertensiven Jugendlichen in den Bereichen der psychosozialen Probleme und Stärken anhand der Elternangaben

Abb.8 Verteilung der Nutzung des Fernsehers (in Stunden pro Tag)

Abb.9 Häufigkeitsverteilung der Nutzung des Computers (in Stunden pro Tag)

Abb.10 Vergleich des Alkoholkonsums zwischen hypertensiven und normotensi- S.57 ven Jugendlichen 


\section{Tabellen}

Tab.1 Items des KINDL-R am Beispiel der Eigenangaben

Tab.2 Items des SDQ am Beispiel der Eigenangaben

Tab.3 Items der Schutzfaktoren am Beispiel der Eigenangaben

Tab.4 Grenzwerte für systolische/diastolische arterielle Hypertonie bei Jungen

Tab.5 Grenzwerte für systolische/diastolische arterielle Hypertonie bei Mädchen

Tab.6 Allgemeine deskriptive Daten für das Gesamtkollektiv sowie für normotensive und hypertensive Jugendliche

Tab.7 Korrelationen (Spearman's Rho) zwischen Eigen- und Elternangaben hinsichtlich der Lebensqualität (KINDL-R)

Tab.8 Korrelationen (Spearman's Rho) zwischen Eigen- und Elternangaben S.39 hinsichtlich psychosozialer Probleme und Stärken

Tab.9 Risiko- und Schutzfaktoren mit Angabe der mittleren Ränge von normotensiven und hypertensiven Jugendlichen sowie dem entsprechenden Signifikanzniveau

Tab.10 Erhöhter arterieller Blutdruck als abhängige Variable im Modell mit den KINDL-R-/SDQ-Variablen (aus den Elternangaben)

Tab.11 Erhöhter arterieller Blutdruck als abhängige Variable im Modell mit den KINDL-R- ISDQ-Variablen (aus den Eigenangaben)

Tab.12 OR und Signifikanz des übergreifenden Messwerts der Lebensqualität S.51 (Elternangaben) sowie der Kontrollvariablen im Modell 1

Tab.13 OR und Signifikanz des Gesamtproblemwerts der psychischen Gesundheit (Elternangaben) sowie der Kontrollvariablen im Modell 1 
Tab.14 Verteilung von Gesundheitsmerkmalen bei hypertensiven und normotensiven Jugendlichen und Signifikanzangabe des Chi-Quadrat-Tests

Tab.15 Verteilung des Alkohol- und Drogenkonsums sowie der körperlichen Aktivität bei hypertensiven und normotensiven Jugendlichen und Signifikanzangabe des Chi-Quadrat-Tests

Tab.16 Angaben zum Allgemeinbefinden im Vergleich zwischen hypertensiven und normotensiven Jugendlichen und Signifikanzangabe des ChiQuadrat-Tests

Tab.17 Übergreifender Messwert der Lebensqualität als abhängige Variable in der multivariaten Testung

Tab.18 Gesamtproblemwert der psychosozialen Schwierigkeiten als abhängige Variable in der multivariaten Testung

\section{Tabellen im Anhang}

Tab.I Kontrollvariablen und Confounder mit OR \& Signifikanz im univariaten und multivariaten Modell am Bsp. des körperlichen Wohlbefindens (Eigenangaben)

Tab.II Kontrollvariablen und Confounder mit OR \& Signifikanz im univariaten und multivariaten Modell am Bsp. Selbstwert (Eigenangaben)

Tab.III Kontrollvariablen und Confounder mit OR \& Signifikanz im bivariaten und multivariaten Modell am Bsp. Familie (Eigenangaben)

Tab.IV Kontrollvariablen und Confounder mit OR \& Signifikanz im univariaten und multivariaten Modell am Bsp. des übergreifenden Messwerts der Lebensqualität (Eigenangaben) 
Tab.V Kontrollvariablen und Confounder mit OR \& Signifikanz im univariaten und multivariaten Modell am Bsp. Hyperaktivität (Eigenangaben)

Tab.VI Kontrollvariablen und Confounder mit OR \& Signifikanz im univariaten und multivariaten Modell am Bsp. körperliches Wohlbefinden (Elternangaben)

Tab.VII Kontrollvariablen und Confounder mit OR \& Signifikanz im univariaten und multivariaten Modell am Bsp. Selbstwert (Elternangaben)

Tab.VIII Kontrollvariablen und Confounder mit OR \& Signifikanz im univariaten und multivariaten Modell am Bsp. Familie (Elternangaben)

Tab.IX Kontrollvariablen und Confounder mit OR \& Signifikanz im univariaten und multivariaten Modell am Bsp. des übergreifenden Messwerts der Lebensqualität (Elternangaben)

Tab.X Kontrollvariablen und Confounder mit OR \& Signifikanz im univariaten und multivariaten Modell am Bsp. emotionale Probleme (Elternangaben)

Tab.XI Kontrollvariablen und Confounder mit OR \& Signifikanz im univariaten und multivariaten Modell am Bsp. Verhaltensprobleme (Elternangaben)

Tab.XII Kontrollvariablen und Confounder mit OR \& Signifikanz im univariaten und multivariaten Modell am Bsp. Hyperaktivität (Elternangaben)

Tab.XIII Kontrollvariablen und Confounder mit OR \& Signifikanz im univariaten und multivariaten Modell am Bsp. des Gesamtproblemwerts (Elternangaben) 


\section{Einleitung}

\subsection{Wachsende klinische Bedeutung der essentiellen arteriellen Hypertonie}

Die arterielle Hypertonie zählt zu den häufigsten chronischen Erkrankungen und ist einer der führenden Gründe für Morbidität und Mortalität weltweit.

Trotz des großen Angebots an effektiven antihypertensiven Medikamenten leiden immer noch viele Patienten an den Langzeitfolgen eines erhöhten Blutdrucks. Die arterielle Hypertonie stellt einen Hauptrisikofaktor für kardiovaskuläre und cerebrovaskuläre Ereignisse dar (Stein et al. 2002; Falkner et al. 2010). Besonders problematisch an dieser weit verbreiteten Erkrankung ist der oftmals lange asymptomatische Verlauf, der plötzlich zu einem lebensbedrohenden Ereignis wie beispielsweise einem Herzinfarkt oder Schlaganfall führen kann. Die Bedeutung der arteriellen Hypertonie als gesundheitlicher Risikofaktor zeigt sich darüber hinaus auch durch die Verursachung von Endorganschäden wie linksventrikulärer Hypertrophie, Herzinsuffizienz, Myokardinfarkt, Nierenerkrankungen, Retinopathie, Schlaganfall und Demenz (Chobanian et al. 2003). Daher muss diese Erkrankung als schwere Belastung für das Gesundheitssystem und die Gesellschaft als Ganzes, aber auch für den einzelnen Patienten (und seine Familie) betrachtet werden. Während die Notwendigkeit von effektiven Möglichkeiten zur Diagnostik und Therapie der essentiellen arteriellen Hypertonie bei Erwachsenen weitgehend akzeptiert ist, wird diesem Problem in der Kindheit und Jugend bisher nur wenig Beachtung geschenkt (Falkner et al. 2010).

Die zeitliche Entfernung zwischen kardiovaskulärem Ereignis im Erwachsenenalter und Blutdruckwerten in der Kindheit erschwert die Feststellung eines Zusammenhangs. Allerdings hat sich gezeigt, dass sich der Ursprung der Erkrankung häufig bis in die Kindheit erstreckt: Es konnte nachgewiesen werden, dass Kinder mit erhöhten Blutdruckwerten eine größere Wahrscheinlichkeit aufweisen, auch als Erwachsene an einer arteriellen Hypertonie zu leiden (Bao et al. 1995; Sun et al. 2007; Falkner et al. 2008; Lurbe et al. 2009). Dies verweist auf die Notwendigkeit, bereits in der Kindheit und Jugend erhöhten Blutdruckwerten die gebührende Aufmerksamkeit zukommen zu lassen. 


\subsection{Essentielle arterielle Hypertonie - bereits bei Kindern und Jugendlichen ein zunehmendes Problem}

Im Gegensatz zu Erwachsenen, für die ein allgemeingültiger Grenzwert für die Definition der arteriellen Hypertonie vorhanden ist, gibt es bei Kindern und Jugendlichen je nach Geschlecht, Alter und Größe viele verschiedene Grenzwerte. Deshalb ist die Diagnose einer arteriellen Hypertonie bei diesem Personenkreis relativ aufwändig und uneindeutig, sodass es oftmals zum „Übersehen“ von Hypertonikern kommt: Hansen et al. (2007) wiesen nach, dass der Blutdruck bei Kindern und Jugendlichen häufig unterdiagnostiziert wird. Nur 26\% der Probanden mit hypertensiven oder "hoch normalen“ Blutdruckwerten erhielten eine korrekte entsprechende Diagnose.

Erschwerend kommt hinzu, dass verschiedene Nationen unterschiedliche Definitionen verwenden: So wird in England beispielsweise nur von einer arteriellen $\mathrm{Hy}$ pertonie gesprochen, wenn der Blutdruckwert oberhalb des 98. Blutdruckperzentils für das entsprechende Alter liegt (Falkner et al. 2010). Am weitesten verbreitet und in den meisten Surveys als Referenz verwendet sind allerdings die Grenzwerte und Definitionen des Fourth Report on the Diagnosis, Evaluation, and Treatment of High Blood Pressure in Children and Adolescents (NHBPEP 2004; Chiolero et al. 2007): Die Definition basiert auf der Normalverteilung der Blutdruckwerte gesunder Kinder und Jugendlicher. Durch die Berücksichtigung von Geschlecht, Alter und Größe wird eine fehlerhafte Klassifikation von besonders kleinen oder besonders großen Kindern und Jugendlichen verhindert. Dadurch ist eine präzisere Einteilung gegeben.

Eine arterielle Hypertonie besteht demnach, wenn der durchschnittliche systolische und/oder diastolische Blutdruck das 95. Perzentil für Geschlecht, Alter und Größe an mindestens drei verschiedenen Messzeitpunkten erreicht oder übersteigt. Der Unterschied zwischen dem 95. und dem 99. Perzentil ist mit 7 bis 10 $\mathrm{mmHg}$ nicht groß genug, um eine adäquate Abgrenzung von milder zu schwerer arterieller Hypertonie treffen zu können, vor allem, wenn die Variabilität der Blutdruckmessung bedacht wird. Daher gibt es zusätzlich die Definition der „arteriellen Hypertonie Stufe 1", die von dem 95. Perzentil bis 5 mmHg oberhalb des 99. Perzentils reicht sowie der „arteriellen Hypertonie Stufe 2“, bei der die Blutdruckwerte $5 \mathrm{mmHg}$ oberhalb des 99. Perzentils liegen (NHBPEP 2004). 
Da sich gezeigt hat, dass hoch normale Blutdruckwerte zu einem erhöhten Risiko führen, eine arterielle Hypertonie zu entwickeln, wurde auch hierfür eine Definition ausgearbeitet. Eine solche „Prähypertension“ liegt vor, wenn die durchschnittlichen systolischen und/oder diastolischen Blutdruckwerte oberhalb des 90., jedoch unterhalb des 95. Perzentils liegen (NHBPEP 2004). Da bei 13- bis 18-Jährigen das 90. Perzentil oftmals oberhalb $120 / 80 \mathrm{mmHg}$ liegt, ist die Bedingung für das Vorliegen einer „Prähypertension“ ebenfalls erfüllt, wenn der Blutdruck zwar unterhalb des 90. Perzentils, aber oberhalb von 120/80 mmHg liegt (NHBPEP 2004; Falkner et al. 2010).

Die weltweite Prävalenz der arteriellen Hypertonie unter Kindern und Jugendlichen ist unbekannt, da die Einschätzung aufgrund verschiedener Definitionen, Messmethoden sowie Art und Umfang des Kollektivs sehr schwierig ist. Die Angaben in verschiedenen Studien reichen von 3\% bis 26\% (Chiolero et al. 2007; McNiece et al. 2007; Bancalari et al. 2011). Insgesamt zeigte sich aber in den vergangenen Jahren eine zunehmende Tendenz, wonach die arterielle Hypertonie bereits in der Kindheit und Jugend ein relevantes Gesundheitsproblem darstellt (Lurbe et al. 2009; Falkner et al. 2010). Mehrfach konnte eine hohe Prävalenz nachgewiesen werden (Muntner et al. 2004; Chiolero et al. 2007; Malatesta-Muncher und Mitsnefes 2012), die besonders häufig bei männlichen Kindern und Jugendlichen auftrat (Din-Dzietham et al. 2007).

In Deutschland gibt es nur wenige Daten, die sich mit der Häufigkeit der arteriellen Hypertonie befassen. Zusätzlich bestehen oftmals Einschränkungen der Aussagekraft, unter anderem in Größe oder Alter des Kollektivs: In einer Studie aus BadenWürttemberg wurde eine Prävalenz von 3\% angegeben. Dabei handelte es sich allerdings um ein relativ kleines Kollektiv von 410 Teilnehmern (Klimm und ReuterKuhn 1994). In einer anderen deutschen Querschnittsstudie wurden neben einer niedrigen Prävalenz einer arteriellen Hypertonie sogar gesunkene durchschnittliche systolische und diastolische Blutdruckwerte im Vergleich zu Ergebnissen aus vorherigen Jahren beschrieben. Da jedoch nur 6-jährige Teilnehmer betrachtet wurden (Haas et al. 2012 b), könnten diese niedrigen Werte auch durch das geringe Durchschnittsalter der Probanden bedingt sein.

Weiterhin kommt es zu Einschränkungen der Aussagekraft der bisher vorliegenden Studien durch die Verwendung von unterschiedlichen Definitionen der arteriel- 
len Hypertonie: Mehrfach wurden bei deutschen Studien an Kindern und Jugendlichen Referenzwerte für Erwachsene zu Grunde gelegt (Neuhauser et al. 2009; Haas et al. 2012 a; Haas et al. 2012 b), was, wie zuvor beschrieben, für dieses Alter jedoch nicht üblich und auch nicht angemessen ist. Die Angaben müssten bei der Berücksichtigung der korrekten Definition einer arteriellen Hypertonie bei Kindern und Jugendlichen wahrscheinlich nach oben korrigiert werden.

Eine andere deutsche Studie verwendete Referenzwerte, die sich von den international weitverbreiteten US-Referenzwerten unterschieden, indem diese ausschließlich anhand normalgewichtiger Kinder und Jugendlicher bestimmt wurden. Dadurch waren die Grenzwerte größtenteils etwas niedriger und es präsentierte sich so eine Prävalenz der arteriellen Hypertonie von 35\% unter adipösen und übergewichtigen Kindern und Jugendlichen (Flechtner-Mors et al. 2011; Neuhauser et al. 2011).

Zusammenfassend ist festzustellen, dass die meisten der vorliegenden Studien auf eine Zunahme der arteriellen Hypertonie bei Kindern und Jugendlichen hinweisen. Die Datenlage erweist sich zur Einschätzung der Prävalenz für Deutschland jedoch als relativ begrenzt.

Im Gegensatz zu Erwachsenen wird bei Kindern und Jugendlichen oft zunächst eine sekundäre arterielle Hypertonie vermutet, wenn erhöhte Blutdruckwerte festgestellt werden. Durch die veränderten Lebensgewohnheiten ist die essentielle arterielle Hypertonie, bei der keine konkrete Ursache gefunden werden kann, jedoch auch bei Jugendlichen zu einem wachsenden Gesundheitsproblem geworden. Verschiedene Untersuchungen befassen sich mit möglichen Einflussfaktoren auf die Prävalenz der arteriellen Hypertonie im Kindes- und Jugendalter Dabei konnte ein deutlicher Zusammenhang mit dem Geschlecht nachgewiesen werden: Männliche Jugendliche wiesen im Vergleich zu weiblichen ein erhöhtes Risiko für eine arterielle Hypertonie auf (Sorof et al. 2004; Din-Dzietham et al. 2007; Kollias et al. 2009). Bezüglich des sozialen Status war der Zusammenhang weniger eindeutig: Zwar zeigte sich bei Erwachsenen mehrfach, dass ein niedriger sozialer Status mit einem höheren kardiovaskulären Risiko einherging (Panagiotakos et al. 2008; Abeyta et al. 2012), dieser Zusammenhang bestätigte sich dagegen bei Kindern und Jugendlichen nicht eindeutig. Neben Studien, die analog zu Erwachsenen einen negativen Einfluss durch einen niedrigen elterlichen Bildungsstatus 
feststellten (Simonetti et al. 2011), wurde andererseits auch eine fehlende Beeinflussung dokumentiert (Katona et al. 2011; Shi et al. 2012).

Weitere Faktoren, die ein gesteigertes kardiovaskuläres Risiko bewirkten, waren rauchende Eltern und eine positive Familienanamnese hinsichtlich einer arteriellen Hypertonie (Munger et al. 1988; Simonetti et al. 2011).

Als Hauptrisikofaktor für eine essentielle arterielle Hypertonie im Jugendalter hat sich die Adipositas herausgestellt (Torrance et al. 2007; Lurbe et al. 2009). In mehreren Studien wurde eine starke lineare Korrelation von BMI und arteriellem Blutdruck festgestellt (Muntner et al. 2004; NHBPEP 2004; Sorof et al. 2004; Falkner et al. 2010; Simonetti et al. 2011; Malatesta-Muncher und Mitsnefes 2012). Bei einer detaillierteren Betrachtung zeigte sich jedoch, dass erst bei einer Überschreitung des 85. BMI-Perzentils ein starker Effekt auf den arteriellen Blutdruck nachweisbar war (Tu et al. 2011). Ausgeprägtes Übergewicht scheint also die Entwicklung einer arteriellen Hypertonie zu begünstigen.

Als potentieller Einflussfaktor auf die steigende Prävalenz der arteriellen Hypertonie gelten außerdem die veränderten Lebensgewohnheiten. Wie bei Erwachsenen koexistierte die arterielle Hypertonie oftmals mit weiteren kardiovaskulären Risikofaktoren, beispielsweise einer schlechten Ernährung oder körperlicher Inaktivität und dafür vermehrter sitzender Tätigkeit (Falkner et al. 2010; Malatesta-Muncher und Mitsnefes 2012). Für die genannten Faktoren gibt es allerdings keinen Nachweis einer direkten Assoziation mit dem arteriellen Blutdruck. Der Zusammenhang mit dem Auftreten von Adipositas ist dagegen belegt und damit zumindest auch die sekundäre Beeinflussung der arteriellen Hypertonie (Kosti und Panagiotakos 2006; Falkner et al. 2010).

Bei Erwachsenen gibt es viele Studien, die die Assoziation von arterieller Hypertonie und körperlicher Inaktivität bestätigten. Bei Kindern und Jugendlichen ist die Anzahl an Untersuchungen zu diesem Thema jedoch sehr eingeschränkt. Die meisten Studien belegten aber, dass regelmäßige körperliche Aktivität den systolischen Blutdruck senken und gegebenenfalls sogar endotheliale Funktionen wiederherstellen kann (Torrance et al. 2007; Lurbe et al. 2009). Dieser positive Effekt durch körperliche Aktivität gilt allerdings nicht für exzessive sportliche Betätigung, für die sogar ein Anstieg des systolischen Blutdrucks nachgewiesen wurde (Tsioufis et al. 2011). 
Obwohl die Datenlage noch eingeschränkt ist, lässt sich bei Kindern und Jugendlichen, ebenso wie bei Erwachsenen, ein verstärkter Alkohol- und Nikotinkonsum als schädigender Einflussfaktor vermuten (Milligan et al. 1997; Flouris et al. 2008; Jorgensen und Maisto 2008; Kollias et al. 2009).

Neben den genannten ungünstigen Lebensgewohnheiten hat sich das Schlafverhalten als blutdruckbeeinflussend herausgestellt. Eine schlechte Schlafqualität steigerte das Risiko für erhöhte Blutdruckwerte um das 4,5-fache, eine zu kurze Schlafdauer um das 2,8-fache (Javaheri et al. 2008).

Zusammenfassend ist festzustellen, dass bei Kindern und Jugendlichen eine Reihe an potentiellen Risikofaktoren für die essentielle arterielle Hypertonie zu vermuten ist, für die es jedoch noch keine umfassende bestätigende Datenlage gibt.

In Anbetracht der steigenden Anzahl hypertensiver Kinder und Jugendlicher erscheint es bedenklich, dass bereits in jungen Jahren Veränderungen von Organstrukturen und -funktionen nachgewiesen werden konnten (Malatesta-Muncher und Mitsnefes 2012). Den am sorgfältigsten dokumentierten Endorganschaden im Jugendalter stellt die linksventrikuläre Hypertrophie dar (NHBPEP 2004; Lurbe et al. 2009). Bereits hoch normale Blutdruckwerte können ungünstige Effekte auf ventrikuläre Strukturen haben (Falkner et al. 2010). So konnte bei $41 \%$ der Jugendlichen mit erhöhten arteriellen Blutdruckwerten eine linksventrikuläre Hypertrophie festgestellt werden (Sorof et al. 2003). Abgesehen von den reinen Strukturveränderungen, die durch erhöhte Blutdruckwerte erzeugt werden, ist die damit assoziierte Steigerung des kardiovaskulären Risikos im Erwachsenenalter besorgniserregend (Lurbe et al. 2009): In einer Untersuchung zeigte sich, dass 17\% der hypertensiven Jugendlichen bereits eine konzentrische Hypertrophie aufwiesen, die mit einem hohen Risiko für kardiovaskuläre Ereignisse assoziiert ist, und $30 \%$ eine exzentrische Hypertrophie, die mit einem mittleren Risiko einhergeht (Daniels et al. 1998). Abgesehen von diesen myokardialen Folgeschäden gilt die arterielle Hypertonie zusätzlich als Hauptrisikofaktor für die Entwicklung einer Herzinsuffizienz (Luma und Spiotta 2006; Lurbe et al. 2009).

Bei Jugendlichen ist die arterielle Hypertonie außerdem mit dem Auftreten einer Mikroalbuminurie assoziiert (Assadi 2008; Lurbe et al. 2009). Beim Erwachsenen zeigt diese die Entwicklung einer diabetischen Nephropathie an, wohingegen die Bedeutung des Auftretens im Rahmen einer arteriellen Hypertonie im Jugendalter 
noch nicht vollständig geklärt ist. Fest steht aber, dass sie ebenfalls einen Marker für eine frühzeitige Endorganschädigung darstellt (Lurbe et al. 2009).

Zusätzlich präsentierte sich ein signifikanter Zusammenhang mit arteriosklerotischen Läsionen in der Aorta und den Koronargefäßen. Die exakten Level und die Dauer der Blutdruckerhöhung, die die Schäden verursachen, sind bei Kindern und Jugendlichen allerdings noch unbekannt (Berenson et al. 1998). Die erste morphologische Veränderung der Arterienwand durch erhöhte Blutdruckwerte ist die Verdickung des Intima-Media-Komplexes, die als Hinweis auf die Entwicklung einer Arteriosklerose gilt (Lurbe et al. 2009). Bei hypertensiven Jugendlichen konnte verstärkt eine arterielle Versteifung durch die Verdickung der Arterienwand festgestellt werden (Sinha und Reid 2007). Dies weist auf die früh einsetzende vaskuläre Schädigung durch eine arterielle Hypertonie hin. Im Zusammenhang damit stehen Schlaganfälle, visuelle Beeinträchtigungen und retinale Gefäßveränderungen sowie die Entwicklung einer hypertensiven Enzephalopathie, die vor allem bei sehr schweren Formen als Komplikation vorkommen können. Zusätzlich wurde eine Assoziation mit einem gehäuften Auftreten von epileptischen Anfällen beobachtet (Luma und Spiotta 2006; Lurbe et al. 2009). Bei Erwachsenen stellen die arterielle Hypertonie und die Dyslipidämie die Hauptrisikofaktoren für die Entwicklung von kognitiven Beeinträchtigungen dar: Hypertensive Erwachsene zeigten eine geringere Aufmerksamkeit, Reaktionszeit und Redegewandtheit. Dies könnte durch arteriosklerotische Veränderungen von kleinen Gefäßen im Gehirn bedingt sein, die zu einer Hypoperfusion, Verlust der Autoregulation und schließlich zu einer Demyelinisierung der weißen Substanz führen (Chobanian et al. 2003). Es gibt einige wenige Studien, die darauf hinweisen, dass auch in der Kindheit geringfügige Veränderungen der kognitiven Funktionen im Rahmen einer arteriellen Hypertonie auftreten (Chobanian et al. 2003; Lande et al. 2009; Falkner et al. 2010). Kinder und Jugendliche mit Blutdruckwerten oberhalb des 90. Perzentils zeigten niedrigere Werte bei kognitiven Tests, insbesondere beim Zahlengedächtnistest. Dies verweist möglicherweise auf eine früh einsetzende Beeinträchtigung des zentralen Nervensystems durch erhöhte arterielle Blutdruckwerte, wobei die potentiellen Effekte der arteriellen Hypertonie auf neuropsychologische Prozesse bei Kindern und Jugendlichen noch sehr begrenzt erforscht sind (Lande et al. 2003). Trotz der eingeschränkten Datenlage zeigt sich, dass bereits im Kindes- und Jugendalter einige pathologische Veränderungen durch erhöhte Blutdruckwerte ver- 
ursacht werden können. Mit zunehmender Erkrankungsdauer ist davon auszugehen, dass diese an Ausmaß und Anzahl zunehmen werden und so zu einer Belastung der Patienten, aber auch des Gesundheitssystems und der Gesellschaft führen werden.

\subsection{Existiert ein Zusammenhang zwischen arterieller Hypertonie und Lebensqualität?}

Im Gegensatz zu den meisten anderen Erkrankungen bleibt die arterielle Hypertonie oftmals für viele Jahre asymptomatisch, vor allem in leichten Stadien. Zum Teil führt sie auch zu unspezifischen Symptomen wie Kopfschmerzen, Schwindel, Müdigkeit sowie kognitiven Beeinträchtigungen und Stimmungsschwankungen (Kjellgren et al. 1998; Erickson et al. 2001), die nicht direkt Rückschlüsse auf die Ursache ermöglichen.

Es wird kontrovers diskutiert, ob eine arterielle Hypertonie mit Veränderungen der Lebensqualität assoziiert ist:

Eine Studie zeigte, dass Hypertoniker ihre Beeinträchtigung durch die arterielle Hypertonie selbst geringer einschätzten als Normotensive und Ärzte. Es scheint als würden Nichterkrankte den Einfluss von arterieller Hypertonie auf die Lebensqualität oftmals überschätzen (Stein et al. 2002). Es konnte sogar nachgewiesen werden, dass unter bestimmten Umständen eine inverse Assoziation des arteriellen Blutdrucks mit selbst wahrgenommenen Problemen $\mathrm{zu}$ bestehen scheint (Winkleby et al. 1988): Probanden mit einem erhöhten arteriellen Blutdruck schätzten im Gegensatz zu den normotensiven Vergleichspersonen ihre berufliche Belastung als weniger stark ein. Eine weitere Studie konnte in einer longitudinalen Untersuchung feststellen, dass Personen, die im Verlauf eine arterielle Hypertonie oder hochnormale Blutdruckwerte entwickelten, selbst von weniger Stressoren, Anspannung, Ängstlichkeit, Depression, Erschöpfung sowie einer größeren beruflichen Zufriedenheit berichteten (Jenkins et al. 1985; Nyklicek et al. 1996).

Die meisten Querschnittsstudien, die sich mit dem Zusammenhang von erhöhtem Blutdruck und Wohlbefinden befassten, beschrieben eine geringere Lebensqualität bei hypertensiven Erwachsenen im Vergleich zu Teilnehmern ohne erhöhte Blutdruckwerte (Battersby et al. 1995; Bardage und Isacson 2001; Erickson et al. 
2001; Klocek und Kawecka-Jaszcz 2003; Li et al. 2005; Arslantas et al. 2008). Neben der beeinträchtigten Lebensqualität zeigte sich bei hypertensiven Erwachsenen auch ein vermehrtes Auftreten von psychischen Problemen, vor allem von emotionalen Störungen. Die Prävalenz einer arteriellen Hypertonie stieg bei Patienten mit Depressionen, Angst- und Panikstörungen stark an (Dimenas et al. 1989; Pickering 2001; Saboya et al. 2010).

Einige wenige Studien fanden jedoch auch keinen Zusammenhang zwischen arterieller Hypertonie und reduziertem Wohlbefinden. Hypertoniker, die nicht über ihre Erkrankung Bescheid wussten, gaben eine ähnlich gute Lebensqualität an wie Normotoniker (Mena-Martin et al. 2003; Barger und Muldoon 2006; Korhonen et al. 2011).

Die widersprüchliche Datenlage wird auch durch eine weitere Untersuchung deutlich, in der sich gegensätzliche Effekte für den systolischen und den diastolischen Blutdruckwert ergaben: Ansteigende systolische Blutdruckwerte waren mit einer schlechteren Lebensqualität assoziiert, ansteigende diastolische dagegen mit einer besseren (Khosravi et al. 2010)

Insgesamt lässt sich somit festhalten, dass die bisherigen Studienergebnisse keine eindeutigen Erkenntnisse in Bezug auf den Zusammenhang von arterieller Hypertonie und Wohlbefinden zulassen, wobei ein Großteil der Studien an Erwachsenen jedoch eine Beeinträchtigung durch erhöhte Blutdruckwerte beschreibt.

Die bisherigen Erkenntnisse über den Zusammenhang von arterieller Hypertonie und Wohlbefinden im Jugendalter sind sehr begrenzt.

Analog zu den Beobachtungen bei Erwachsenen beschrieben vereinzelte Untersuchungen auch bei Jugendlichen eine verstärkte Assoziation erhöhter Blutdruckwerte mit vermehrten psychischen Problemen: So konnten Lernschwierigkeiten und ein Aufmerksamkeitsdefizit-Hyperaktivitätssyndrom (ADHS) häufiger bei hypertensiven als bei normotensiven Jugendlichen festgestellt werden. Die verstärkten Probleme beim Lernen waren unabhängig von der Einnahme von Stimulanzien zur ADHS-Therapie (Adams et al. 2010), sodass eine medikamentös induzierte sekundäre Blutdruckerhöhung unwahrscheinlich ist.

In einer anderen Studie konnten bei jugendlichen Hypertonikern verstärkt internalisierende Probleme festgestellt werden. Sie zeigten höhere Rohwerte in den Bereichen „Ängstlichkeit“, „Verschlossenheit", „soziale Probleme“ und „Aufmerksam- 
keitsprobleme" sowie bei den DSM-orientierten Skalen in den Bereichen „Angststörungen“, „Aufmerksamkeitsdefizit/Hyperaktivitätsprobleme“ und „Oppositionelltrotzige Störungen“. Eine signifikante Assoziation war jedoch vor allem zwischen Angst bzw. Depression und einer arteriellen Hypertonie zu beobachten. Der Entstehungsmechanismus hierfür blieb jedoch unklar. Zudem handelte es sich um ein sehr kleines Kollektiv von nur je 32 Normo- und Hypertonikern (Lande et al. 2009), weshalb eine allgemeingültige Aussage über den Zusammenhang von arteriellem Blutdruck und Wohlbefinden nur sehr begrenzt möglich ist.

Eine weitere Untersuchung wies für verschiedene psychische Probleme unterschiedliche Blutdrucktendenzen nach: Depressive Symptome korrelierten für beide Geschlechter positiv mit dem Blutdruck, wohingegen Symptome einer Angststörung nur bei männlichen Teilnehmern positiv mit dem arteriellen Blutdruck korrelierten. Für Verhaltensstörungen zeigte sich bei Jungen dagegen eine negative Korrelation (Rogeness et al. 1990), das heißt ein zu den beiden internalisierenden Störungen gegensätzlicher Trend. Neben der stark begrenzten Datenlage ist es die Uneindeutigkeit der Beobachtungen, die die Interpretation des Zusammenhangs von arterieller Hypertonie und Wohlbefinden vor allem im Jugendalter erschwert.

Aufgrund der steigenden Anzahl an Hypertonikern wächst jedoch das Interesse, diese chronische Erkrankung besser zu verstehen. Zusätzlich zu den kardiovaskulären Korrelaten sollten auch die psychosomatischen Aspekte einer arteriellen Hypertonie näher untersucht werden. Dies wäre für die Entwicklung weiterer Präventions- und Interventionsmöglichkeiten von großer Bedeutung. 


\subsection{Zielsetzung dieser Arbeit}

Angesichts der einerseits zunehmenden epidemiologischen Bedeutung der Hypertonie im Kindes- und Jugendalter und der andererseits unbefriedigenden Datenlage zu Prävalenz, prädisponierenden Faktoren und potentiellen Auswirkungen eines erhöhten arteriellen Blutdrucks soll mit der vorliegenden Arbeit dazu beigetragen werden, diese Lücke bezogen auf den Personenkreis der älteren Kinder und Jugendlichen in Deutschland zu schließen.

Aufgrund der eingeschränkten Datenlage soll die Prävalenz und Verteilung erhöhter Blutdruckwerte genauer an einem großen, für Deutschland repräsentativen Kollektiv untersucht werden. Dabei sollen, um eine Vergleichbarkeit mit anderen Studien zu ermöglichen, international gebräuchliche Referenzwerte für den Blutdruck verwendet werden.

Weiterhin soll verstärkt auf prädisponierende Faktoren mit speziellem Fokus auf psychosomatische und kardiovaskuläre Gesichtspunkte eingegangen werden. Der Personenkreis der 11- bis 17-Jährigen wurde gewählt, da sich in diesem Alter besondere Belastungen aufgrund der Pubertät als mögliche zusätzliche begünstigende Faktoren für die Entwicklung einer Hypertonie vermuten lassen.

Um die pathophysiologischen Mechanismen der ansteigenden Prävalenz der arteriellen Hypertonie in den letzten Jahrzehnten besser zu verstehen, ist es von großer Wichtigkeit, die Lebensgewohnheiten der Jugendlichen zu identifizieren, die mit erhöhten Blutdruckwerten assoziiert sind. Es soll untersucht werden, ob es Unterschiede zwischen normotensiven und hypertensiven Jugendlichen bei kardiovaskulären Risikofaktoren gibt. Weiterhin sollen potentielle Differenzen hinsichtlich weiterer Gesundheitsparameter dargestellt werden. Die zu betrachtenden kardiovaskulären Korrelate umfassen daher die folgenden Fragestellungen:

- Welche kardiovaskulären Risikofaktoren weisen hypertensive Jugendliche auf?

- Welche Unterschiede bestehen zwischen hyper- und normotensiven Jugendlichen im Freizeitverhalten?

- Sind bei hypertensiven Jugendlichen bereits gehäuft gesundheitliche oder kognitive Beeinträchtigungen zu bemerken? 
In Bezug auf die psychosomatischen Korrelate sollen insbesondere die psychische Gesundheit, die Lebensqualität sowie die Schutzfaktoren der Jugendlichen in den Mittelpunkt der Untersuchung gestellt werden. Bisher existieren nur sehr wenige Studien, die sich mit dem Zusammenhang eines erhöhten Blutdrucks und Wohlbefinden ausschließlich bei Kindern und Jugendlichen befassen. Allerdings ist bei innen das Ausmaß an hypertensiven Folgeschäden und Beeinträchtigungen höchstwahrscheinlich geringer als bei Erwachsenen, die meist bereits an einer lang andauernden chronischen Hypertonie leiden. Daher sollte anhand des weniger beeinflussten Kollektivs der Jugendlichen überprüft werden, ob ein erhöhter arterieller Blutdruck zu einer Minderung des Wohlbefindens führt. Die folgenden Fragestellungen sollen daher bearbeitet werden:

- Gibt es Unterschiede zwischen normotensiven und hypertensiven Jugendlichen in Bezug auf personale, soziale und familiäre Schutzfaktoren?

- Sind in Abhängigkeit des Blutdrucks Unterschiede bei psychosozialen Problemen festzustellen?

- Leiden hypertensive Jugendliche, wie Erwachsene, an einer eingeschränkten Lebensqualität? Welche Bereiche sind hier besonders betroffen?

\subsection{Hypothesen zum erhöhten arteriellen Blutdruck im Jugend- alter}

\subsubsection{Hypothesen zur Prävalenz}

- Bei Jugendlichen in Deutschland zeigt sich eine hohe Prävalenz erhöhter arterieller Blutdruckwerte.

- Mit zunehmendem Alter steigt die Anzahl betroffener Jugendlicher.

- Jungen sind häufiger von einem erhöhten arteriellen Blutdruck betroffen als Mädchen. 


\subsubsection{Hypothesen bezüglich kardiovaskulärer Risikofaktoren}

- Adipositas im Jugendalter korreliert stark mit dem Vorhandensein eines erhöhten arteriellen Blutdrucks.

- Eine geringe körperliche Fitness zeigt eine Assoziation mit einem erhöhten arteriellen Blutdruck.

- Bei einem gesteigerten Genuss von Alkohol und Zigaretten zeigen sich häufiger erhöhte Blutdruckwerte.

- Ein ausgeprägter Medienkonsum ist häufiger bei hypertensiven Jugendlichen zu finden.

- Hypertensive Jugendliche nehmen häufiger Medikamente ein als normotensive.

- Hypertensive Jugendliche weisen häufiger zusätzliche Erkrankungen auf.

- Hypertensive Jugendliche zeigen (durch kognitive Beeinträchtigungen) schlechtere Schulleistungen.

\subsubsection{Hypothesen bezüglich psychosomatischer Korrelate}

- Ein hohes Maß an personalen Schutzfaktoren ist mit einem geringeren Auftreten erhöhter arterieller Blutdruckwerte assoziiert.

- Jugendliche mit einer guten sozialen und familiären Unterstützung weisen seltener einen erhöhten arteriellen Blutdruck auf.

- Emotionale Probleme wie Ängste und depressive Verstimmungen gehen häufiger mit erhöhten arteriellen Blutdruckwerten einher.

- Verhaltensprobleme und Hyperaktivität finden sich vermehrt bei hypertensiven Jugendlichen.

- Jugendliche mit einem geringen seelischen und körperlichen Wohlbefinden weisen häufiger erhöhte Blutdruckwerte auf.

- Jugendliche mit einem geringen Selbstwertgefühl leiden häufiger an einem erhöhten arteriellen Blutdruck. 
- Ein geringes Wohlbefinden im sozialen Umfeld (Schulstress, Konflikte im Elternhaus und mit Freunden) zeigt eine Assoziation mit erhöhten Blutdruckwerten. 


\section{Methodik}

\subsection{Beschreibung der KiGGS-Studie}

Der Kinder- und Jugendgesundheitssurvey (KiGGS-Studie) ist eine deutschlandweite vom Robert-Koch-Institut organisierte Querschnittstudie zum Gesundheitszustand von Kindern und Jugendlichen. Gefördert wurde die Studie vom Bundesministerium für Gesundheit sowie vom Bundesministerium für Bildung und Forschung. Der von Mai 2003 bis Mai 2006 durchgeführte Befragungs- und Untersuchungssurvey hatte zum Ziel, umfangreiche bevölkerungsrepräsentative Daten für die Kinder und Jugendlichen im Alter von 0 bis 17 Jahren in Deutschland zu erheben (Kamtsiuris et al. 2007).

Die Daten der Studie wurden an 167 repräsentativ ausgewählten Städten und Gemeinden in Deutschland erhoben, wobei die Auswahl der Untersuchungsorte und der Probanden mit Hilfe des Zentrums für Umfragen, Methoden und Analysen (ZUMA) in Mannheim getroffen wurde (Hölling et al. 2007 b). Pro Altersgruppe sollten Informationen zu ungefähr 1000 Jungen und Mädchen erhoben werden (Kurth 2007), um insgesamt eine Gesamtteilnehmerzahl von knapp 18.000 zu erreichen. Die Auswahl der Zielpersonen erfolgte anhand der Einwohnermelderegister. Insgesamt nahmen mit einer Teilnehmerzahl von 17.641 zwei Drittel der 26.899 eingeladenen Kinder und Jugendlichen an der Studie teil, was einer hohen Responsequote von 66,6\% entspricht (Kamtsiuris et al. 2007).

Für die Beurteilung der Repräsentativität der Stichprobe muss ein Vergleich der gesundheitsbezogenen Merkmale zwischen Teilnehmern und Nichtteilnehmern der Studie möglich sein. Um zu überprüfen, ob sich diese in wichtigen Punkten unterscheiden, wurde ein Kurzfragebogen für die Nichtteilnehmer eingesetzt, der Eckdaten zum allgemeinen Gesundheitszustand und zum Sozialstatus erhebt (Kurth et al. 2002).

Die KiGGS-Studie weist einen modularen Aufbau auf, bei dem der Kernsurvey Befragungen und Untersuchungen $\mathrm{zu}$ den wichtigsten gesundheitsrelevanten Themenbereichen des Kindes-und Jugendalters umfasst. An Unterstichproben der Studienteilnehmer können zusätzlich inhaltlich vertiefende Befragungen und Un- 
tersuchungen durchgeführt werden als von Kooperationspartnern finanzierte und organisierte Module (Kurth et al. 2002). Zusätzliche Module gibt es in den Bereichen Ernährung, Umwelt, Motorik und psychische Gesundheit sowie in einem Ländermodul Schleswig-Holstein und in einem Jodmonitoring (Kurth 2007).

Die Durchführung des Kernstudie erfolgte durch vier ärztlich geleitete Teams, bestehend aus Arzt, Zentrumsinterviewer, Untersucher, MTA und Umweltinterviewer (Hölling et al. 2007 b). Für die Untersuchung des Kollektivs wurde eine Einteilung in fünf Altersbereiche (0-2 Jahre, 3-6 Jahre, 7-10 Jahre, 11-13 Jahre und 14-17 Jahre) vorgenommen, um durch die Berücksichtigung alters-, entwicklungs- und verhaltensspezifischer Merkmale eine differenzierte Betrachtung zu ermöglichen (Kurth et al. 2002). Es erfolgte eine schriftliche Befragung der Eltern sowie der Kinder ab 11 Jahren durch selbst auszufüllende Fragebögen, wobei zwei unterschiedliche altersangepasste Fragebögen zum Einsatz kamen (Kurth et al. 2002). Um möglichst umfassende und vergleichbare Daten zu erheben, wurden die Selbst- und Fremdeinschätzungsbögen inhaltlich angeglichen (Kurth et al. 2002). Vor Beginn der Untersuchung erklärte der Zentrumsinterviewer die Handhabung des Fragebogens eingehend, und es wurde darauf geachtet, dass Eltern und Jugendliche die Fragen unabhängig voneinander beantworteten. Das ärztliche Interview erfolgte als laptopgestützte standardisierte Befragung zu speziellen Krankheiten, der Medikamentenanwendung der letzten sieben Tage und dem Impfstatus. Die körperliche Untersuchung sollte objektive Messdaten zu wesentlichen Gesundheitsproblemen im Kindes- und Jugendalter liefern (Hölling et al. 2007 b). Qualitätskriterien für die KiGGS-Studie waren die Einhaltung des Datenschutzes und die Einhaltung ethischer Grundsätze, was durch den Bundesbeauftragten für Datenschutz und die Ethikkommission des Virchowklinikums der Berliner Humboldt-Universität geprüft wurde (Kurth 2007). Sämtliche Studienteilnehmer und ihre Erziehungsberechtigten gaben ihre Einverständniserklärung zur Teilnahme an dem Kinder- und Jugendgesundheitssurvey.

Die Verständlichkeit und die praktische Anwendbarkeit der entwickelten Fragebögen wurde während eines einjährigen Pretests an den Studienteilnehmer getestet, um gegebenenfalls Verbesserungen durch veränderte Frageformulierungen zu erzeugen (Kamtsiuris et al. 2002; Kurth 2007). Bei der Erprobung der Erhebungsinstrumente sollte erhoben werden, inwieweit sie praktikabel waren, wie viel Zeit die Probanden für das Ausfüllen benötigten und inwiefern der Fragenkatalog ihre 
Zustimmung fand. Dazu wurden die Fragebögen in Zusammenarbeit mit der ZUMA einem Cognitive Debriefing unterzogen, wobei die Teilnehmer in der ersten Phase aufgefordert wurden, ihre Antworten näher zu kommentieren. In der zweiten Phase wurden die Probanden hinsichtlich Zeitdauer, Reaktionen, Problemen und Auffälligkeiten beim Ausfüllen der Fragebögen beobachtet. Zuletzt wurden die Teilnehmer gebeten, den Fragebogen zu benoten. Die Auswertung der Ergebnisse erfolgte unter besonderer Berücksichtigung von Validität und Plausibilität. Insgesamt zeigte sich im Pretest eine gute Akzeptanz der Fragebögen, die durchschnittlich mit der Schulnote 2,5 beurteilt wurden. Als Ergebnis wurden die Fragebögen um 15-20\% gekürzt, einzelne Formulierungen geändert sowie Fragen herausgenommen (Kamtsiuris et al. 2002).

Insgesamt liegt mit der KiGGS-Studie nun ein repräsentativer, umfassender Datensatz vor, an dem die Gesundheit und das Wohlbefinden der Kinder- und Jugendlichen genauer betrachtet werden kann.

\subsection{Blutdruckmessung im Kinder- und Jugendgesundheits- survey}

Die Blutdruckmessung erfolgte durch ein automatisches Blutdruckmessgerät (Datascope Accutorr Plus), das ein oszillometrisches Verfahren verwendet (Mindray 2007). Der arterielle Mitteldruck wird dabei aufgrund gemessener Pulswellenoszillationen bestimmt. Aus diesem berechnet das Gerät nach einem bestimmten Algorithmus den systolischen und diastolischen Blutdruckwert (Babbs 2012). Die drei Blutdruckwerte mit der Pulsfrequenz werden digital angezeigt (Neuhauser und Thamm 2007).

Die Messung wurde im Sitzen vorgenommen, nach einer fünfminütigen Ruhezeit sowie nach einem körperlich wenig anstrengenden Untersuchungsteil (Sehtest). Insgesamt wurden zwei Messungen in einem Abstand von einer zweiminütigen Ruhepause durchgeführt.

Die Messung fand mit wenigen Ausnahmen am rechten Arm statt, der standardisiert in Herzhöhe und unbekleidet gelagert werden sollte. Es standen vier verschiedene Größen der originalen Datascope-Blutdruckmanschetten zur Verfügung $(6 \times 12 \mathrm{~cm}, 9 \times 18 \mathrm{~cm}, 12 \times 23 \mathrm{~cm}, 17 \times 38,6 \mathrm{~cm})$. Durch den Untersucher sollte die Man- 
schettengröße so gewählt werden, dass mindestens zwei Drittel des Oberarms (gemessen von Axilla bis Ellenbogenfalte) bedeckt waren. Die Markierung der Manschette sollte sich über der zuvor palpierten Arteria brachialis befinden (Neuhauser und Thamm 2007).

\subsection{Bestimmung der Lebensqualität}

Die gesundheitsbezogene Lebensqualität wurde multidimensional mit dem krankheitsübergreifenden KINDL-R-Fragebogen erfasst (Ravens-Sieberer 2000). Der ursprünglich von Bullinger et al. (1994) entwickelte und anschließend von RavensSieberer und Bullinger (1998) revidierte KINDL-R-Fragebogen ist ein originär deutschsprachiges Instrument zur Erfassung der Lebensqualität von Kindern und Jugendlichen im Alter von 4 bis 17 Jahren, das bei klinischen Populationen aber auch bei gesunden Kindern und Jugendlichen Anwendung finden kann (Schumacher et al. 2003). Durch den mehrdimensionalen Aufbau sollen die Aspekte der Lebensqualität berücksichtigt werden, die sich für die Gruppe der Kinder und Jugendlichen als relevant herausgestellt haben (Schumacher et al. 2003). Der revidierte KINDL-R umfasst 24 Items, die sechs Dimensionen der Lebensqualität mit jeweils vier Items im Rückblick auf die letzte Woche erfassen. Dazu zählen: das körperliche und emotionale Wohlbefinden, der Selbstwert, das familiäre und schulische Wohlbefinden sowie das Wohlbefinden in Bezug auf Freunde/Gleichaltrige (Ravens-Sieberer et al. 2007 a). In den verschiedenen Versionen des KINDL-R-Fragebogens werden altersspezifische Veränderungen in den Dimensionen der Lebensqualität berücksichtigt (Schumacher et al. 2003). Für die vorliegende Untersuchung relevant waren der Kid-KINDL für 8- bis 12-jährige Kinder sowie der Kiddo-KINDL für 13- bis 16-jährige Jugendliche (Ravens-Sieberer 2000). Der Fragebogen ist sowohl in der Selbstberichtsversion als auch in der Elternversion verfügbar (Ravens-Sieberer 2000).

Die Items konnten anhand von fünfstufigen Antwortmöglichkeiten ("nie“, „selten“, „manchmal“, „oft", „immer") beantwortet werden (Ravens-Sieberer et al. 2007 a). Dabei erhielt "nie" den Wert 1 und „immer" den Wert 5. Pro Skala wurde ein Summenwert gebildet, wobei für einige Items zunächst eine Umpolung der Antwortkategorien erfolgte (Schumacher et al. 2003). Anschließend wurden die Werte auf 
eine Skala von 0 bis 100 transformiert (Ravens-Sieberer und Bullinger 1998). Höhere Werte gehen dabei mit einer besseren Lebensqualität einher. Aus den 24 Items konnte ein übergreifender Messwert der gesundheitsbezogenen Lebensqualität berechnet werden, der ebenfalls auf einer Skala von 0 bis 100 angegeben wurde (Ravens-Sieberer et al. 2007 a).

Am Beispiel der Selbstbeurteilungsversion der 14- bis 17- Jährigen soll anhand einer Tabelle 1 ein Überblick über die einzelnen Items verschafft werden.

Tab. 1 Items des KINDL-R am Beispiel der Eigenangaben

\begin{tabular}{|c|c|c|c|c|c|}
\hline $\begin{array}{c}\text { Körperliches } \\
\text { Wohlbefinden }\end{array}$ & $\begin{array}{c}\text { Emotionales } \\
\text { Wohlbefinden }\end{array}$ & Selbstwert & $\begin{array}{c}\text { Familiäres } \\
\text { Wohlbefin- } \\
\text { den }\end{array}$ & $\begin{array}{c}\text { Wohlbefin- } \\
\text { den mit } \\
\text { Freunden }\end{array}$ & $\begin{array}{c}\text { Schulisches } \\
\text { Wohlbefin- } \\
\text { den }\end{array}$ \\
\hline Krankheitsgefühl & $\begin{array}{c}\text { Lachen, Spaß } \\
\text { haben }\end{array}$ & Stolz & $\begin{array}{c}\text { mit Eltern gut } \\
\text { verstehen }\end{array}$ & $\begin{array}{c}\text { Unterneh- } \\
\text { mung mit } \\
\text { Freunden }\end{array}$ & $\begin{array}{c}\text { gute Bewälti- } \\
\text { gung der Auf- } \\
\text { gaben }\end{array}$ \\
\hline Schmerzen & Langeweile & Wohlfühlen & $\begin{array}{c}\text { zu Hause } \\
\text { wohlfühlen }\end{array}$ & $\begin{array}{c}\text { "gut ankom- } \\
\text { men“ }\end{array}$ & $\begin{array}{c}\text { Interesse am } \\
\text { Unterricht }\end{array}$ \\
\hline $\begin{array}{c}\text { Müdigkeit, Er- } \\
\text { schöpfung }\end{array}$ & $\begin{array}{c}\text { Gefühl des } \\
\text { Alleinseins }\end{array}$ & $\begin{array}{c}\text { sich selbst } \\
\text { mögen }\end{array}$ & Streit & "gut verste- \\
hen“ & $\begin{array}{c}\text { Zukunftssor- } \\
\text { gen }\end{array}$ \\
\hline Kraft, Ausdauer & $\begin{array}{c}\text { Ängstlichkeit, } \\
\text { Unsicherheit }\end{array}$ & $\begin{array}{c}\text { gute Ideen } \\
\text { haben }\end{array}$ & $\begin{array}{c}\text { Einschrän- } \\
\text { kungen durch } \\
\text { Eltern }\end{array}$ & $\begin{array}{c}\text { Andefühl des } \\
\text { Anseins }\end{array}$ & $\begin{array}{c}\text { Angst vor } \\
\text { schlechten } \\
\text { Noten }\end{array}$ \\
\hline
\end{tabular}

\subsection{Bestimmung der psychosozialen Probleme und Stärken}

Anzeichen für psychosoziale Probleme und Stärken wurden anhand des Strengths and Difficulties Questionnaire (SDQ) erfasst (Goodman 1997). Der SDQ liegt als Selbstbeurteilungsversion für Kinder und Jugendliche ab 11 Jahren vor sowie als Fremdbeurteilungsversion für Eltern von Kindern ab drei Jahren (Hölling et al. 2007 a). Die deutschsprachige Version ist seit 1997 verfügbar (Klasen et al. 2000). Bei der Auswahl der Items wurden die Klassifikationssysteme DSM-IV (American Psychiatric Association 1994) und ICD-10 (World Health Organisation 1994) beachtet (Goodman und Scott 1999). 
Der SDQ umfasst 25 Items, wovon sich 10 auf Stärken beziehen, 14 auf Schwächen und eines neutral ist (,besser mit Erwachsenen auskommen als mit Gleichaltrigen“) (Goodman 1997). Das Screening-Instrument umfasst fünf Skalen mit jeweils fünf Items: emotionale Probleme, Verhaltensprobleme, Hyperaktivität, Probleme mit Gleichaltrigen und prosoziales Verhalten (Goodman und Scott 1999).

Jedes Item konnte mit einer dreistufigen Antwortmöglichkeit beantwortet werden (,trifft nicht zu“ "trifft teilweise zu“ „trifft eindeutig zu“). Die Skalierung der Antworten erfolgte mit 0 für „trifft nicht zu“, 1 für "trifft teilweise zu“ und 2 für „trifft eindeutig zu“. Bei fünf Items, die in der nachfolgenden Tabelle kursiv gedruckt sind, war die Skalierung umgekehrt: 2 für "trifft nicht zu“, 1 für "trifft teilweise zu“ und 0 für "trifft eindeutig zu“ (Goodman et al. 1998). Der Score für die jeweilige Skala ergab sich durch die Summierung der Zahlenwerte der zugehörigen Items.

Aus den Bereichen emotionale Probleme, Verhaltensprobleme, Hyperaktivität und Probleme mit Gleichaltrigen konnte ein Gesamtproblemwert berechnet werden, der Werte von 0 bis 40 umfasste. Der Wert des prosozialen Verhaltens floss dabei nicht in den Gesamtproblemwert mit ein, da die Abwesenheit von prosozialem Verhalten nicht mit dem Vorliegen von psychischen Auffälligkeiten gleichzusetzen ist (Goodman et al. 1998). Am Beispiel der Selbstbeurteilungsversion der 13- bis 17- Jährigen soll anhand der Tabelle 2 ein Überblick über die einzelnen Items verschafft werden. 
Tab. 2 Items des SDQ am Beispiel der Eigenangaben

\begin{tabular}{|c|c|c|c|c|}
\hline $\begin{array}{c}\text { Emotionale } \\
\text { Probleme }\end{array}$ & $\begin{array}{l}\text { Verhaltens- } \\
\text { probleme }\end{array}$ & Hyperaktivität & $\begin{array}{l}\text { Probleme mit } \\
\text { Gleichaltrigen }\end{array}$ & $\begin{array}{l}\text { Prosoziales } \\
\text { Verhalten }\end{array}$ \\
\hline $\begin{array}{l}\text { häufige Kopf- oder } \\
\text { Bauchschmerzen } \\
\text { haben; oft schlecht } \\
\text { werden }\end{array}$ & $\begin{array}{l}\text { leicht wütend } \\
\text { werden; oft Be- } \\
\text { herrschung ver- } \\
\text { lieren }\end{array}$ & $\begin{array}{l}\text { oft unruhig sein; } \\
\text { nicht lange still- } \\
\text { sitzen können }\end{array}$ & $\begin{array}{l}\text { meistens allein } \\
\text { sein; mit sich } \\
\text { selbst beschäfti- } \\
\text { gen }\end{array}$ & $\begin{array}{l}\text { nett zu anderen } \\
\text { Menschen sein; } \\
\text { ihre Gefühle } \\
\text { sind einem wich- } \\
\text { tig }\end{array}$ \\
\hline $\begin{array}{c}\text { sich häufig Sorgen } \\
\text { machen }\end{array}$ & $\begin{array}{c}\text { normalerweise } \\
\text { das tun, was } \\
\text { gesagt wird }\end{array}$ & $\begin{array}{l}\text { dauernd in Be- } \\
\text { wegung sein; } \\
\text { zappelig }\end{array}$ & $\begin{array}{l}\text { einen oder meh- } \\
\text { rere gute Freun- } \\
\text { de/-innen } \\
\text { haben }\end{array}$ & $\begin{array}{l}\text { mit anderen } \\
\text { teilen }\end{array}$ \\
\hline $\begin{array}{l}\text { oft unglücklich/ } \\
\text { niedergeschlagen; } \\
\text { häufig weinen }\end{array}$ & $\begin{array}{c}\text { sich häufig } \\
\text { schlagen; ande- } \\
\text { re zwingen zu } \\
\text { tun, was man } \\
\text { will }\end{array}$ & $\begin{array}{c}\text { leicht ablenken } \\
\text { lassen; schwer } \\
\text { sich zu konzent- } \\
\text { rieren }\end{array}$ & $\begin{array}{l}\text { im Allgemeinen } \\
\text { bei Gleichaltri- } \\
\text { gen beliebt sein }\end{array}$ & $\begin{array}{c}\text { hilfsbereit, wenn } \\
\text { andere verletzt, } \\
\text { krank oder trau- } \\
\text { rig }\end{array}$ \\
\hline $\begin{array}{c}\text { neue Situationen } \\
\text { machen nervös; } \\
\text { Verlust des Selbst- } \\
\text { vertrauens }\end{array}$ & $\begin{array}{l}\text { andere behaup- } \\
\text { ten oft, dass } \\
\text { man lüge oder } \\
\text { mogele }\end{array}$ & $\begin{array}{l}\text { nachdenken be- } \\
\text { vor man handelt }\end{array}$ & $\begin{array}{c}\text { von anderen } \\
\text { gehänselt oder } \\
\text { schikaniert wer- } \\
\text { den }\end{array}$ & $\begin{array}{l}\text { nett zu jüngeren } \\
\text { Kindern sein }\end{array}$ \\
\hline $\begin{array}{l}\text { viele Ängste haben, } \\
\text { sich leicht fürchten }\end{array}$ & $\begin{array}{l}\text { Dinge nehmen, } \\
\text { die einem nicht } \\
\text { gehören }\end{array}$ & $\begin{array}{c}\text { Angefangenes } \\
\text { wird zu Ende } \\
\text { gemacht; man } \\
\text { kann sich lang } \\
\text { genug konzent- } \\
\text { rieren }\end{array}$ & $\begin{array}{l}\text { besser mit Er- } \\
\text { wachsenen aus- } \\
\text { kommen als mit } \\
\text { Gleichaltrigen }\end{array}$ & $\begin{array}{l}\text { anderen oft frei- } \\
\text { willig helfen }\end{array}$ \\
\hline
\end{tabular}

\subsection{Bestimmung der Schutzfaktoren}

Anhand eines kurzen mehrdimensionalen Untersuchungsinstruments sollten die Schutzfaktoren der psychischen Gesundheit von Kindern und Jugendlichen erfasst werden (Bettge und Ravens-Sieberer 2003). Informationen zu den drei Dimensionen personale, soziale und familiäre Ressourcen wurden mit Hilfe von insgesamt 22 Items erhoben. Am Ende des Abschnitts werden die verschiedenen Items tabellarisch dargestellt (Tabelle 3, S. 23).

\subsubsection{Personale Ressourcen}

Die personalen Schutzfaktoren wurden bei den 11- bis 17-Jährigen im Eigenbericht anhand von fünf Items erfasst (Erhart et al. 2007). Drei Items stammen aus 
der Selbstwirksamkeitsskala von Schwarzer und Jerusalem (Schwarzer und Jerusalem 1999). Sie messen die Überzeugung der Kinder und Jugendlichen, inwiefern sie in der Lage sind, schwierige Anforderungen bewältigen zu können (Bettge und Ravens-Sieberer 2003). Ein Item stammt aus der Optimismusskala des Berner Fragebogens zum Wohlbefinden (Grob et al. 1991) und ein Item gehört zur Sense of Coherence Scale (Kern et al. 1995).

Die Fragen konnten durch vier Antwortmöglichkeiten („stimmt nicht“, „stimmt kaum“, stimmt eher“, „stimmt genau“) beantwortet werden (Erhart et al. 2007). Den Antwortkategorien wurden Zahlenwerte von 1 bis 4 zugeordnet, wobei „stimmt nicht“ den Wert 1 und „stimmt genau“ den Wert 4 erhielt. Die Werte der einzelnen Items wurden summiert und anschließend in eine Skala, die von 0 bis 100 reicht, umgewandelt.

\subsubsection{Soziale Ressourcen}

Die sozialen Ressourcen der Kinder und Jugendlichen wurden anhand von acht Items erfragt, die aus der deutschen Übersetzung der Social Support Scale stammen (Donald und Ware 1984; Erhart et al. 2007). Im Eigenbericht wurden die Kinder und Jugendlichen zur Häufigkeit von sozialer Unterstützung in verschiedenen Situationen befragt (Bettge und Ravens-Sieberer 2003).

Die einzelnen Items konnten mit fünfstufigen Antwortmöglichkeiten („nie“, „selten“, „manchmal“, „oft", „immer") beantwortet werden. Die Kodierung der Werte erfolgte mit Werten von 1 für die Antwort „nie“ bis 5 für die Antwort „immer“. Für die Bildung des Scores wurden die einzelnen Items summiert und anschließend auf eine Skala von 0 bis 100 transformiert, wobei höhere Werte mit einer besseren sozialen Unterstützung einhergehen (Erhart et al. 2007). 


\subsubsection{Familiäre Ressourcen}

Die familiären Ressourcen wurden anhand von neun Items bestimmt, die aus einer gekürzten Version der Familienklima-Skala stammen (Schneewind et al. 1985; Erhart et al. 2007). Im Selbst- sowie im Elternbericht konnten die entsprechenden Fragen mit vier verschiedenen Antwortkategorien („stimmt nicht“, „stimmt kaum“, „stimmt eher“, „stimmt genau“) beantwortet werden. Die Kodierung der Werte erfolgte hier mit 1 für „stimmt nicht“ bis 4 für „stimmt genau“ (Erhart et al. 2007). Bei zwei Items, die in der nachfolgenden Tabelle kursiv gedruckt sind, erfolgte eine Umpolung der Werte. Die einzelnen Werte der Items wurden auch hier aufsummiert und anschließend auf eine Skala von 0 bis 100 transformiert.

\section{Tab.3 Items der Schutzfaktoren am Beispiel der Eigenangaben}

\begin{tabular}{|c|c|c|}
\hline Personale Ressourcen & Soziale Ressourcen & Familiäre Ressourcen \\
\hline \multirow[b]{2}{*}{$\begin{array}{c}\text { Was auch passiert, ich } \\
\text { werde schon klarkommen. }{ }^{1)}\end{array}$} & Gibt es jemanden,... & $\begin{array}{l}\text { In unserer Familie geht } \\
\text { jeder auf die Sorgen und } \\
\text { Nöte des anderen ein. }\end{array}$ \\
\hline & $\begin{array}{l}\text { der dir zuhört, wenn du das } \\
\text { Bedürfnis nach einem Ge- } \\
\text { spräch hast? }\end{array}$ & $\begin{array}{c}\text { Am Wochenende geht es } \\
\text { bei uns zu Hause häufig } \\
\text { ziemlich eintönig und lang- } \\
\text { weilig zu. }\end{array}$ \\
\hline \multirow[b]{2}{*}{$\begin{array}{l}\text { Für jedes Problem kann ich } \\
\text { eine Lösung finden. }{ }^{1)}\end{array}$} & $\begin{array}{l}\text { der dir Liebe und Zunei- } \\
\text { gung zeigt? }\end{array}$ & $\begin{array}{l}\text { Wir kommen wirklich alle } \\
\text { gut miteinander aus. }\end{array}$ \\
\hline & $\begin{array}{l}\text { mit dem du zusammen } \\
\text { Spaß haben kannst? }\end{array}$ & $\begin{array}{l}\text { Wir gehen oft ins Kino, be- } \\
\text { suchen Sportveranstaltun- } \\
\text { gen oder machen Ausflüge. }\end{array}$ \\
\hline $\begin{array}{l}\text { Wenn ein Problem auf- } \\
\text { taucht, kann ich es aus } \\
\text { eigener Kraft meistern. }{ }^{1)}\end{array}$ & $\begin{array}{l}\text { der dir Informationen gibt, } \\
\text { um dir beim Verstehen ei- } \\
\text { ner Situation zu helfen? }\end{array}$ & $\begin{array}{l}\text { Bei allem, was wir zu Hau- } \\
\text { se tun, sind wir mit Begeis- } \\
\text { terung dabei. }\end{array}$ \\
\hline \multirow{2}{*}{$\begin{array}{c}\text { Meine Zukunft sieht gut } \\
\text { aus. }^{2)}\end{array}$} & der dich umarmt? & $\begin{array}{l}\text { Bei uns vergeht kein Wo- } \\
\text { chenende, ohne dass wir } \\
\text { etwas unternehmen. }\end{array}$ \\
\hline & $\begin{array}{l}\text { mit dem zusammen du dich } \\
\text { entspannen kannst? }\end{array}$ & $\begin{array}{l}\text { In unserer Familie hat jeder } \\
\text { das Gefühl, dass man ihm } \\
\text { zuhört und auf ihn eingeht. }\end{array}$ \\
\hline \multirow{2}{*}{$\begin{array}{l}\text { Die Dinge, die ich jeden } \\
\text { Tag mache, bereiten mir } \\
\text { Freude und sind lustig. }{ }^{3)}\end{array}$} & $\begin{array}{c}\text { mit dem du etwas unter- } \\
\text { nehmen kannst, um dich } \\
\text { abzulenken? }\end{array}$ & $\begin{array}{l}\text { Abends und an den Wo- } \\
\text { chenenden unternehmen } \\
\text { wir selten etwas, sondern } \\
\text { bleiben lieber zu Hause. }\end{array}$ \\
\hline & $\begin{array}{l}\text { der dich liebt und der dir } \\
\text { das Gefühl gibt, geliebt und } \\
\text { gebraucht zu werden? }\end{array}$ & $\begin{array}{l}\text { Bei uns ist man eher groß- } \\
\text { zügig, wenn bestimmte } \\
\text { Dinge nicht so hundertpro- } \\
\text { zentig gemacht werden. }\end{array}$ \\
\hline
\end{tabular}

1) Selbstwirksamkeitsskala

3) Sense of Coherence Scale 


\subsection{Andere untersuchte Merkmale}

Der soziale Status wurde anhand der Elternangaben für beide Elternteile erhoben, wobei dem Haushalt der höhere Indexscore zugewiesen wurde. Bei getrennt lebenden Eltern erhielt das Kind den Score des Elternteils, bei dem es die meiste Zeit lebt (Lange et al. 2007). Für die Berechnung des mehrdimensionalen Indexscores wurden Daten zur Schulbildung und beruflichen Qualifikation, zur beruflichen Stellung sowie zum Haushaltsnettoeinkommen erhoben (Jöckel et al. 1998; Lampert et al. 2002). Die Beantwortung erfolgte auf einer Skala bestehend aus sieben Kategorien mit zugewiesenen Punktewerten von eins bis sieben. Der Indexwert des sozialen Status ergab sich durch Summation der drei Skalenwerte, wodurch Werte von 3 bis 21 angenommen werden konnten (Winkler und Solzenberg 1999). Anhand des erreichten Scores wurde eine Einteilung in drei Statusgruppen vorgenommen: „niedriger Sozialstaus“ (3-8 Punkte), „mittlerer Sozialstatus“ (9-14 Punkte), „hoher Sozialstatus“ (15-21 Punkte) (Winkler und Solzenberg 1999; Lampert und Kroll 2006).

Zum Thema Gesundheit wurde der subjektive Gesundheitsstatus der Jugendlichen anhand der Eigenangaben erhoben. Die fünfstufige Antwortskala reichte dabei von „sehr gut“ bis „sehr schlecht". Weiterhin wurde erfragt, ob ein Aufmerksamkeitsdefizit/Hyperaktivitätssyndrom (ADHS) durch einen Arzt oder Psychologen diagnostiziert wurde. Anhand der deutschen Übersetzung des CSHCN (Children with Special Health Care Needs) wurde erfasst, ob ein spezieller Versorgungsbedarf bei den Jugendlichen vorlag. Dieser sollte anhand der individuellen funktionellen Beeinträchtigung oder der Inanspruchnahme medizinischer Leistungen bestimmt werden (Scheidt-Nave et al. 2007). Anhand der Elternangaben wurde durch drei Hauptfragen erhoben, ob medizinische oder nichtmedizinische Leistungen benötigt oder beansprucht wurden. Dazu gehörten die Einnahme von Medikamenten oder die Inanspruchnahme bzw. der Bedarf an medizinischer, psychosozialer oder pädagogischer Unterstützung oder speziellen Therapien wie Physio-, Ergo- oder Sprachtherapie. Zwei weitere Fragen befassten sich mit bestehenden funktionellen Einschränkungen oder emotionalen, Entwicklungs- oder Verhaltensproblemen (Bethell et al. 2002; Scheidt-Nave et al. 2007). Zusätzlich konnte die Frage nach weiteren Erkrankungen dichotom mit „ja“ oder „nein“ beantwortet werden. Beim Allgemeinbefinden wurden die Jugendlichen im Selbstein- 
schätzungsbogen nach Schlafschwierigkeiten wie Ein- und Durchschlafschwierigkeiten befragt, die sie ebenfalls mit „ja“ oder "nein“ beantworten konnten. Die Frage nach dem Schuldurchlauf konnte mit drei verschiedenen Antwortmöglichkeiten „alle Klassen wurden regulär durchlaufen“, „eine/mehrere Klasse(n) wurden übersprungen“ oder „eine/mehrere Klasse(n) wurden wiederholt" beantwortet werden. Beim Freizeitverhalten wurden die Eigenangaben der Jugendlichen hinsichtlich des Konsums von Medien, Alkohol, Zigaretten und Drogen sowie zur körperlichen Aktivität und der körperlichen Leistungsfähigkeit betrachtet: Bei den Medien stand eine fünfstufige Antwortskala („gar nicht“, „0,5 Stunden“, „1-2 Stunden“, „3-4 Stunden“, „mehr als vier Stunden“) zur Verfügung, mit der die Jugendlichen den zeitlichen Umfang der Nutzung von Fernseher, Computer und Handy angeben konnten. Beim Alkoholkonsum wurde zunächst dichotom erfasst, ob bisher jemals Alkohol konsumiert wurde. Anschließend stand eine 7-stufige Antwortskala zur Verfügung („1 oder mehr Gläser pro Tag“, „5-6 Gläser pro Woche“, „2-4 Gläser pro Woche“, „1 Glas pro Woche“, „1-3 Gläser pro Monat", „weniger als 1 Glas pro Monat", „gar nicht"), mit der der Verzehr von Bier, Wein und Schnaps genauer quantifiziert werden konnte. Die Frage nach dem Rauchen konnte mit "ja“ oder "nein“ beantwortet werden. Eine fünf-stufige Antwortmöglichkeit mit „nie“, „einmal“, „mehrmals“, „oft“ oder „unbekannt" stand für die Angaben zum Drogenkonsum zur Verfügung.

Außerdem wurden anhand von Eigenangaben die körperliche Aktivität (,jeden Tag“, „3-5 mal pro Woche“, „1-2 mal pro Woche“, „1-2 mal pro Monat") und die körperliche Leistungsfähigkeit (,sehr gut“, „gut“, „mittel“, ,nicht besonders gut“, „gar nicht gut") erfasst.

\subsection{Statistische Auswertungen}

\subsubsection{Allgemeine Angaben}

Als Datengrundlage der folgenden Berechnungen diente der vom Robert-KochInstitut zur Verfügung gestellte Public Use File, der sämtliche Daten der Basiserhebung des Kinder- und Jugendgesundheitssurveys (2003-2006) beinhaltet. 
Die statistischen Analysen erfolgten mit SPSS-18 (PASW 18). Die Berechnungen wurden mit einem Gewichtungsfaktor durchgeführt, damit repräsentative Aussagen für Kinder und Jugendliche in Deutschland getroffen werden konnten (Kamtsiuris et al. 2007). Auf diese Weise sollten Unterschiede zwischen der NettoStichprobe und der Bevölkerungsstruktur in Deutschland (Stand: 31.12.2004) in Bezug auf Alter (in Jahren), Geschlecht, Region (Ost/West/Berlin) und Staatsangehörigkeit korrigiert werden (Ravens-Sieberer et al. 2007 a). Für die Berechnung des Gewichtungsfaktors wurde zunächst ein Designgewicht bestimmt, das umgekehrt proportional zur Auswahlwahrscheinlichkeit der Teilnehmer war. Diese Auswahlwahrscheinlichkeit ergab sich aus dem Produkt der Auswahlwahrscheinlichkeit der Gemeinde und der Auswahlwahrscheinlichkeit des Probanden innerhalb der Gemeinde. Außerdem wurde ein Anpassungsgewicht erzeugt, das Abweichungen der designgewichteten Netto-Stichprobe von der Bevölkerungsstruktur (Stand: 31.12.2004) im Hinblick auf Alter, Geschlecht, Region und Staatsangehörigkeit ausgleichen sollte. Der endgültige Gewichtungsfaktor ergab sich aus dem Produkt des Designgewichts und dem Anpassungsgewicht. Zuletzt erfolgte eine Normierung des Gewichts, um die Summe der Gewichte wieder dem Stichprobenumfang anzugleichen (Kamtsiuris et al. 2007).

\subsubsection{Definition der Blutdruckvariablen}

Für die Definition der Blutdruck-Variablen wurden die Blutdrucklevel der National High Blood Pressure Education Programm (NHBPEP) Working Group on High Blood Pressure in Children and Adolescents zugrunde gelegt (NHBPEP 2004). Es wurden Grenzwerte für einen systolischen bzw. diastolischen erhöhten arteriellen Blutdruck entsprechend dem 95. Blutdruckperzentils je nach Geschlecht, Alter und Größenperzentil erstellt (siehe Tabelle 4 und 5). Den Körpergrößenangaben der Probanden wurden Größenperzentile zugeordnet. Entsprechend dem Mittelwert aus der ersten und zweiten arteriellen Blutdruckmessung wurden sowohl der systolische als auch der diastolische Blutdruckwert in „normal“ versus „erhöht“ unterteilt und drei dichotome Variablen erzeugt: „systolischer Blutdruck“ (0=normaler systolischer Blutdruckwert; 1=systolischer Blutdruckwert $\geq 95$.Perzentil); „diastolischer Blutdruck" (0=normaler diastolischer Blutdruckwert; 1=diastolischer Blut- 
druckwert $\geq 95$.Perzentil); „erhöhter arterieller Blutdruck“ ( $0=$ normale systolische und diastolische Blutdruckwerte; 1=systolischer und/oder diastolischer Blutdruckwert $\geq 95$. Perzentil).

Tab. 4 Grenzwerte für systolische/diastolische arterielle Hypertonie bei Jungen (NHBPEP 2004)

\begin{tabular}{|c|c|c|c|c|c|c|c|c|c|c|c|c|c|c|c|}
\hline \multicolumn{2}{|c|}{ Jungen } & \multicolumn{7}{|c|}{$\begin{array}{l}\text { Systolischer Blutdruck } \\
\text { (mmHg) }\end{array}$} & \multicolumn{7}{|c|}{$\begin{array}{l}\text { Diastolischer Blutdruck } \\
(\mathrm{mmHg})\end{array}$} \\
\hline \multirow{2}{*}{$\begin{array}{l}\text { Alter } \\
\text { in Jah- } \\
\text { ren }\end{array}$} & \multirow{2}{*}{$\begin{array}{l}\text { BD- } \\
\text { Perz. }\end{array}$} & \multicolumn{7}{|c|}{ Größenperzentil } & \multicolumn{7}{|c|}{ Größenperzentil } \\
\hline & & 5. & 10. & 25. & 50. & 75. & 90. & 95. & 5. & 10. & 25. & 50. & 75. & 90. & 95. \\
\hline 11 & 95. & 117 & 118 & 119 & 121 & 123 & 124 & 125 & 78 & 78 & 79 & 80 & 81 & 82 & 82 \\
\hline 12 & 95. & 119 & 120 & 122 & 123 & 125 & 127 & 127 & 78 & 79 & 80 & 81 & 82 & 82 & 83 \\
\hline 13 & 95. & 121 & 122 & 124 & 126 & 128 & 129 & 130 & 79 & 79 & 80 & 81 & 82 & 83 & 83 \\
\hline 14 & 95. & 124 & 125 & 127 & 128 & 130 & 132 & 132 & 80 & 80 & 81 & 82 & 83 & 84 & 84 \\
\hline 15 & 95. & 126 & 127 & 129 & 131 & 133 & 134 & 135 & 81 & 81 & 82 & 83 & 84 & 85 & 85 \\
\hline 16 & 95. & 129 & 130 & 132 & 134 & 135 & 137 & 137 & 82 & 83 & 83 & 84 & 85 & 86 & 87 \\
\hline 17 & 95. & 131 & 132 & 134 & 136 & 138 & 139 & 140 & 84 & 85 & 86 & 87 & 87 & 88 & 89 \\
\hline
\end{tabular}


Tab. 5 Grenzwerte für systolische/diastolische arterielle Hypertonie bei Mädchen (NHBPEP 2004)

\begin{tabular}{|c|c|c|c|c|c|c|c|c|c|c|c|c|c|c|c|}
\hline \multicolumn{2}{|c|}{ Mädchen } & \multicolumn{7}{|c|}{$\begin{array}{l}\text { Systolischer Blutdruck } \\
\text { (mmHg) }\end{array}$} & \multicolumn{7}{|c|}{$\begin{array}{l}\text { Diastolischer Blutdruck } \\
(\mathrm{mmHg})\end{array}$} \\
\hline \multirow{2}{*}{$\begin{array}{c}\text { Alter } \\
\text { in Jah- } \\
\text { ren }\end{array}$} & \multirow{2}{*}{$\begin{array}{l}\text { BD- } \\
\text { Perz. }\end{array}$} & \multicolumn{7}{|c|}{ Größenperzentil } & \multicolumn{7}{|c|}{ Größenperzentil } \\
\hline & & 5. & 10. & 25. & 50. & 75. & 90. & 95. & 5. & 10. & 25. & 50. & 75. & 90. & 95. \\
\hline 11 & 95. & 118 & 118 & 119 & 121 & 122 & 123 & 124 & 78 & 78 & 78 & 79 & 80 & 81 & 81 \\
\hline 12 & 95. & 119 & 120 & 121 & 123 & 124 & 125 & 126 & 79 & 79 & 79 & 80 & 81 & 82 & 82 \\
\hline 13 & 95. & 121 & 122 & 123 & 124 & 126 & 127 & 128 & 80 & 80 & 80 & 81 & 82 & 83 & 83 \\
\hline 14 & 95. & 123 & 123 & 125 & 126 & 127 & 129 & 129 & 81 & 81 & 81 & 82 & 83 & 84 & 84 \\
\hline 15 & 95. & 124 & 125 & 126 & 127 & 129 & 130 & 131 & 82 & 82 & 82 & 83 & 84 & 85 & 85 \\
\hline 16 & 95. & 125 & 126 & 127 & 128 & 130 & 131 & 132 & 82 & 82 & 83 & 84 & 85 & 85 & 86 \\
\hline 17 & 95. & 125 & 126 & 127 & 129 & 130 & 131 & 132 & 82 & 83 & 83 & 84 & 85 & 85 & 86 \\
\hline
\end{tabular}

\subsubsection{Beschreibung des Kollektivs}

Für Gruppenvergleiche wurde zum einen der t-Test für unabhängige Stichproben angewendet, um die Gruppe der normotensiven und die Gruppe der hypertensiven Jugendlichen auf Unterschiede in der Verteilung des Alters, des Gewichts und des $B M I$ zu überprüfen. Anhand des Chi-Quadrat-Tests sollten mögliche Unterschiede bezüglich der Verteilung des Geschlechts und des sozialen Status nachgewiesen werden. Die Testung fand zum Signifikanzniveau von $\alpha=5 \%$ statt. 


\subsection{4 Überprüfung der Korrelation}

Die Korrelation zwischen den Elternangaben und den Eigenangaben bezüglich der Lebensqualität und den psychischen Auffälligkeiten und Stärken wurde durch den Rangkorrelationskoeffizienten nach Spearman (Spearman-Rho) bestimmt. Dieser wurde ebenfalls für die Überprüfung der Korrelation zwischen den Subskalen der Lebensqualität und dem übergreifenden Messwert sowie für die Subskalen der psychischen Auffälligkeiten und dem Gesamtproblemwert verwendet. Die Signifikanz wurde zum Niveau von $\alpha=5 \%$ getestet.

2.7.5 Überprüfung der Verteilung von Lebensqualität, psychosozialen Problemen und Stärken sowie Schutzfaktoren bei normotensiven und hypertensiven Jugendlichen

Anschließend sollten die beiden Gruppen auf Unterschiede in der Verteilung von Lebensqualität (KINDL-R), psychosozialen Problemen und Stärken (SDQ) sowie von Schutzfaktoren überprüft werden. Hierbei wurden die Eigen- sowie die Elternangaben berücksichtigt. Zum nichtparametrischen Vergleich der zwei unabhängigen Stichproben wurde der U-Test nach Mann und Whitney verwendet. Auch hier galt das Signifikanzniveau von $\alpha=5 \%$.

Die Variablen der Lebensqualität, der psychosozialen Probleme und Stärken sowie der Schutzfaktoren, die im U-Test nach Mann und Whitney signifikant unterschiedlich zwischen den Gruppen waren, sollten mittels der binären logistischen Regression weiter untersucht werden. Als Kovariablen für das multivariate Modell dienten das Alter, das Geschlecht, der BMI und der soziale Status. Als Messgröße für die Stärke des Zusammenhangs zwischen der abhängigen Variablen „erhöhter arterieller Blutdruck“ und den oben genannten unabhängigen Variablen wurden die verschiedenen Odds Ratios mit Signifikanz betrachtet. Zunächst wurde die binäre logistische Regression im univariaten Modell für jede unabhängige Variable einzeln durchgeführt. Anschließend erfolgte die Testung im Modell 1, in dem jede unabhängige Variable jeweils mit den Variablen Alter, Geschlecht, BMI und sozia- 
lem Status als Kovariablen überprüft wurde. Die Testung fand auch hier zum Signifikanzniveau von $\alpha=5 \%$ statt.

Die Güte der Modellanpassung wurde durch die Likelihood-Funktion bestimmt, für die als Maß der negative doppelte Wert des Logarithmus (-2LL) gilt. Bei einer optimalen Modellanpassung würde der Funktionswert (-2LL) den Wert 0 annehmen. Außerdem konnten der Klassifizierungstabelle die Prozentwerte für die „richtig negativ“ und „richtig positiv“ geschätzten Fälle entnommen werden.

\subsubsection{Explorative Testung der normotensiven und hypertensiven Ju-} gendlichen auf weitere Unterschiede

Um die Gruppe der Normotensiven und der Hypertensiven explorativ auf weitere bestehende Unterschiede zu überprüfen, wurde der Chi-Quadrat-Test verwendet. Die untersuchten Variablen wurden im Abschnitt 2.6 genauer erläutert. Die Testung der genannten Variablen fand zum Signifikanzniveau von $\alpha=5 \%$ statt.

Die unabhängigen Variablen, die sich als signifikant unterschiedlich zwischen den Gruppen erwiesen, wurden mittels der binären logistischen Regression multivariat mit den Variablen „Alter“, „Geschlecht“, „BMI“ und „sozialer Status“ überprüft. Es wurden die Odds Ratios mit Signifikanz betrachtet.

\subsubsection{Multivariate Testung im Modell 1b}

Zuletzt sollten die Subskalen der Lebensqualität und der psychosozialen Probleme und Stärken jeweils einzeln im multivariaten Modell 1b mit dem Alter, dem Geschlecht, dem BMI und dem sozialen Status sowie den explorativ gewonnenen Confoundern überprüft werden. Dabei wurden für die binäre logistische Regression nur die Confounder im Modell $1 \mathrm{~b}$ berücksichtigt, die sich im Chi-Quadrat-Test als signifikant unterschiedlich erwiesen. Bei der Auswertung der Daten zu Einschränkungen im Alltag bzw. Bierkonsum wurden fehlende Angaben definiert als Nichtvorhandensein des entsprechenden Parameters. Als Messgröße für die Stär- 
ke des Zusammenhangs zwischen der Variablen „erhöhter arterieller Blutdruck“ und den unabhängigen Variablen wurden die Odds Ratios mit Signifikanz betrachtet.

\subsubsection{Lebensqualität und psychosoziale Probleme als abhängige Vari- ablen}

Anhand der linearen Regression sollte überprüft werden, ob der arterielle Blutdruck in der multivariaten Testung als Prädiktor für Lebensqualität und psychosoziale Schwierigkeiten betrachtet werden kann. Dabei sollte für Alter, Geschlecht, $B M I$ und sozialen Status adjustiert werden. Bei der Lebensqualität wurde der übergreifende Messwert verwendet. Es konnten Werte von 0 bis 100 angenommen werden. Bei den psychosozialen Problemen wurde der Gesamtproblemwert benutzt, der Werte von 0 bis 40 annehmen konnte. BMI und Alter flossen als metrische Variablen ein. Der soziale Status wurde als ordinale Variable mit den Ausprägungen niedriger, mittlerer und hoher Sozialstatus erfasst. Der arterielle Blutdruck wurde als dichotome Variable, d.h. systolisch und/oder diastolisch erhöhter arterieller Blutdruck verwendet. Die Testung fand zum Signifikanzniveau von $\alpha=5 \%$ statt. Zur Beurteilung der Anpassungsgüte des Modells wurde das adjustierte R-Quadrat betrachtet, das Werte zwischen 0 und 1 annehmen kann. Der BetaKoeffizient als standardisierter Regressionskoeffizient gibt den Einfluss der unabhängigen Variablen an (Bühl 2010). 


\section{Ergebnisse}

\subsection{Beschreibung des Kollektivs und der Häufigkeitsverteilung eines erhöhten arteriellen Blutdrucks}

Das Kollektiv der 11- bis 17-Jährigen, das während der KiGGS-Studie untersucht wurde, weist insgesamt eine Anzahl von 7697 Teilnehmern auf. Blutdruckmesswerte sind dabei für 7688 von innen vorhanden. Die Geschlechterverteilung ist dabei annähernd ausgeglichen: 51,3\% der Probanden sind männlich und 48,7\% weiblich.

Insgesamt haben 10,7\% des oben beschriebenen Kollektivs einen systolischen und/oder diastolischen Blutdruckwert oberhalb des 95. Perzentils. Bezüglich der Geschlechtsverteilung ist ein Unterschied festzustellen, da insgesamt $12,8 \%$ der männlichen Teilnehmer und nur 8,5\% der weiblichen einen erhöhten systolischen oder diastolischen Blutdruckwert aufweisen.

Mit zunehmendem Alter zeigen die männlichen Teilnehmer einen wachsenden Anteil an erhöhten systolischen oder diastolischen Blutdruckwerten. Bei den 11Jährigen sind es noch 5,8\%, wohingegen es bei den 17-Jährigen schon $18 \%$ sind. Bei den Mädchen ist mit zunehmendem Alter kein wachsender Anteil an erhöhten Blutdruckwerten festzustellen: Die Werte schwanken zwischen 8 und 9\% mit einem minimalen Anteil von 7,3\% bei den 17-Jährigen und einem maximalen Anteil von $11,4 \%$ bei den 12-Jährigen.

Das nachfolgende Balkendiagramm (Abbildung 1) zeigt die Häufigkeit von normotensiven und hypertensiven Jugendlichen in Prozent getrennt nach Alter und Geschlecht. 


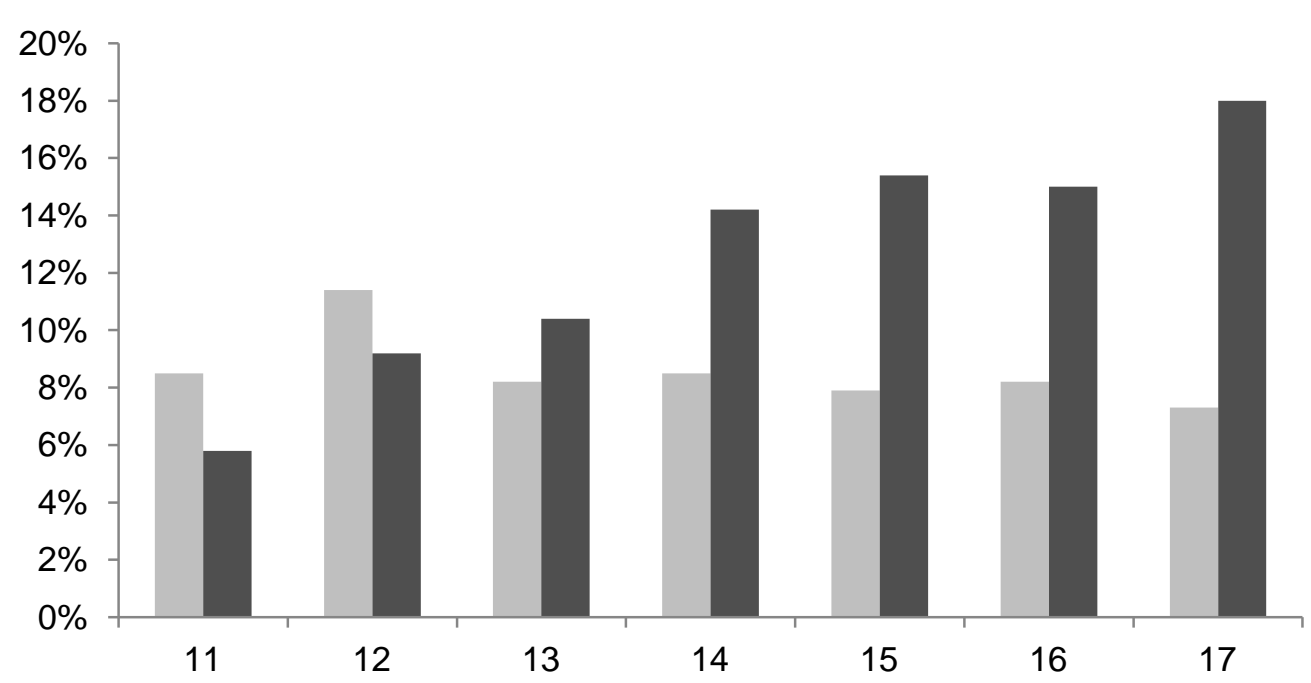

\section{Abb. 1 Häufigkeitsverteilung des erhöhten arteriellen Blutdrucks in Ab- hängigkeit von Alter und Geschlecht}

Altersabhängige Prävalenz von erhöhten systolischen und/oder diastolischen Blutdruckmessungen bei männlichen (dunkel grau) und weiblichen (grau) Studienteilnehmern des KiGGS-Survey.

Bei einer getrennten Betrachtung von systolischen und diastolischen Blutdruckwerten zeigt sich, dass der Anteil der Kinder und Jugendlichen mit erhöhten systolischen Blutdruckwerten mit insgesamt 9,7\% deutlich höher liegt als der Anteil mit erhöhten diastolischen Blutdruckwerten, der sich insgesamt auf 2,7\% beläuft. Bezüglich der Geschlechtsverteilung sind sowohl bei den systolischen als auch bei den diastolischen Blutdruckwerten die männlichen Teilnehmer mit 11,8\% und $3,1 \%$ stärker betroffen als die weiblichen Teilnehmerinnen mit einem Anteil von 7,6\% bzw. 2,4\%.

Bei der Betrachtung der systolischen Blutdruckwerte in den verschiedenen Altersgruppen zeigen die männlichen Teilnehmer mit zunehmendem Alter einen wachsenden Anteil an systolischen Blutdruckwerten oberhalb des 95. Perzentils (siehe Abbildung 2): Bei den 11-Jährigen sind es noch 5,0\%, wohingegen es bei den 17Jährigen schon $16,5 \%$ sind. Bei den Mädchen ist mit zunehmendem Alter kein steigender Anteil an erhöhten systolischen Blutdruckwerten festzustellen. Der Anteil der 11-jährigen Mädchen mit systolischen Blutdruckwerten oberhalb des 95. Perzentils liegt mit 7,1\% etwas höher als bei den gleichaltrigen Jungen. Die Al- 
tersklasse mit dem größten Anteil zeigt sich mit 10,6\% bei den 12-Jährigen, ansonsten variieren die Anteile zwischen 6 und 8\%.

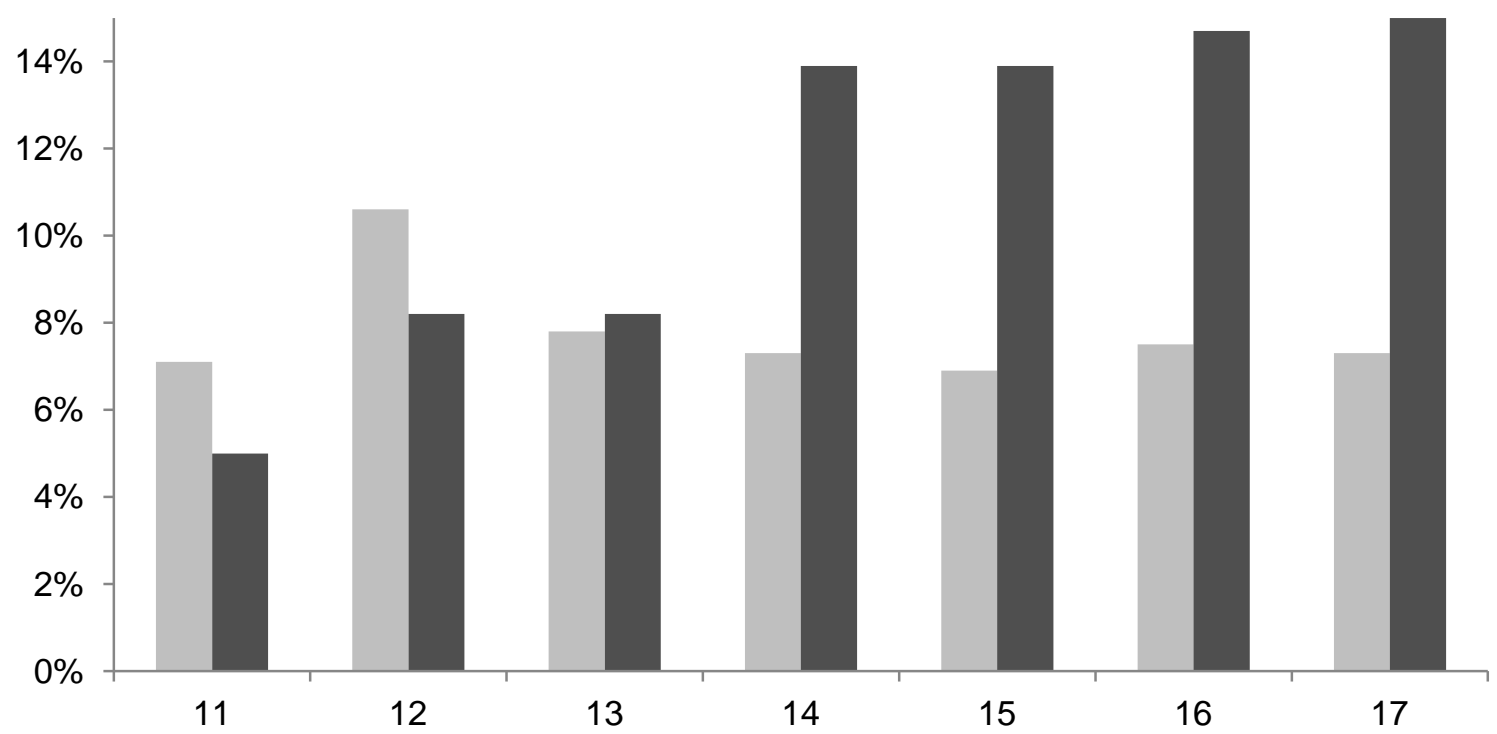

Abb. 2 Häufigkeitsverteilung des erhöhten systolischen Blutdrucks in Abhängigkeit von Alter und Geschlecht

Altersabhängige Prävalenz von erhöhten systolischen Blutdruckmessungen bei männlichen (dunkel grau) und weiblichen (grau) Studienteilnehmern des KiGGS-Survey.

Bei der Betrachtung der diastolischen Blutdruckwerte (Abbildung 3) zeigen die männlichen Teilnehmer Anteile an erhöhten Blutdruckwerten, die zwischen 2 und 4\% schwanken. Mit 1,9\% weisen die 11-Jährigen den kleinsten Anteil auf, die 16Jährigen mit 4\% dagegen den größten Anteil.

Bei den Mädchen schwankt der Anteil mit erhöhten diastolischen Blutdruckwerten zwischen 2 und 3\%. Den kleinsten Anteil zeigen die 13-Jährigen mit 1,7\%, den größten Anteil die 16-Jährigen mit 3,1\%. 


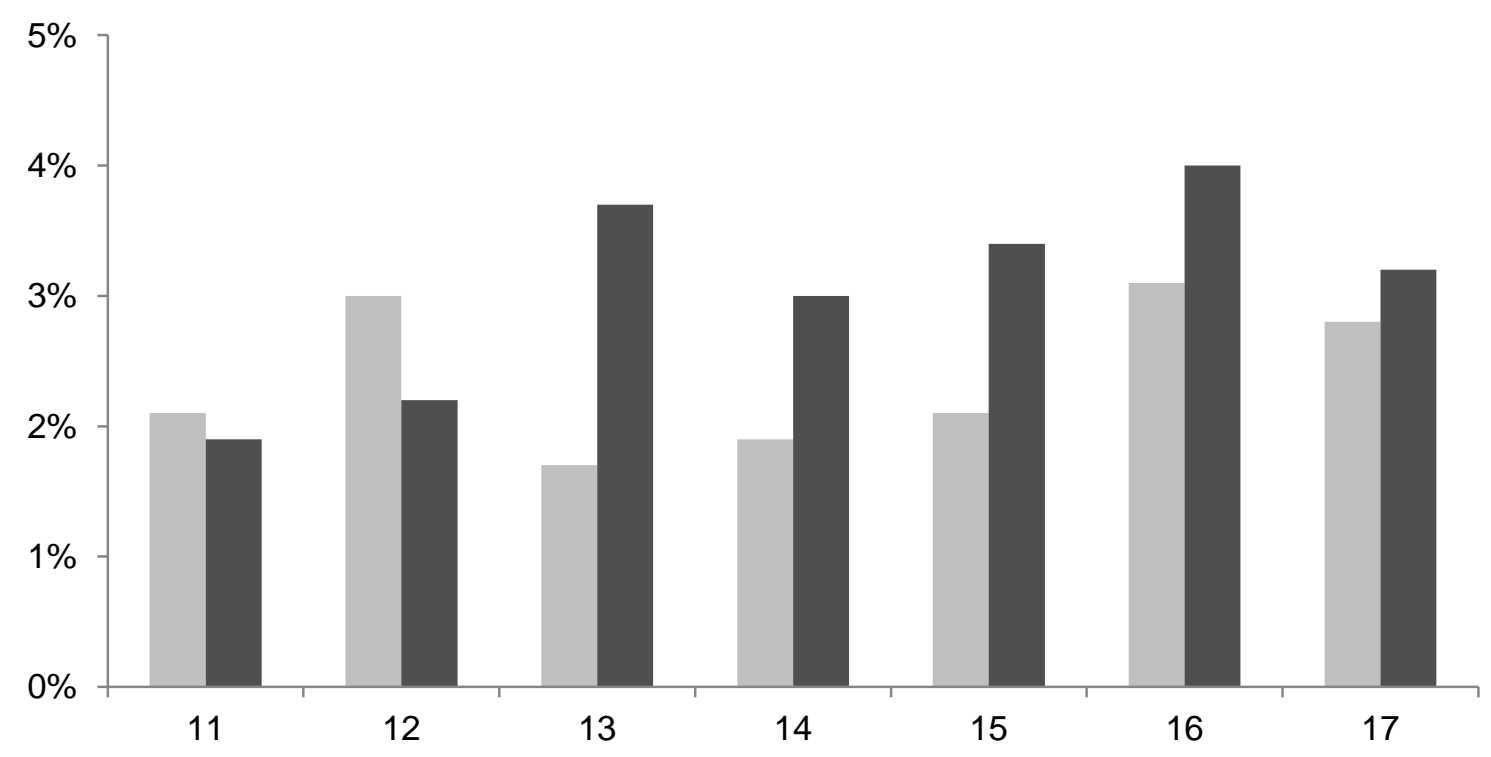

\section{Abb. 3 Häufigkeitsverteilung des erhöhten diastolischen Blutdrucks in Abhängigkeit von Alter und Geschlecht}

Altersabhängige Prävalenz von erhöhten diastolischen Blutdruckmessungen bei männlichen (dunkel grau) und weiblichen (grau) Studienteilnehmern des KiGGS-Survey.

Für die Auswertung wurde eine Gruppendichotomisierung anhand des systolischen und des diastolischen Blutdruckwertes vorgenommen. Die eine Gruppe umfasst Probanden mit normalen systolischen und diastolischen Blutdruckwerten, die andere diejenigen mit systolischen und/oder diastolischen Blutdruckwerten oberhalb des 95. Perzentils.

Die Gruppe mit normalen Blutdruckwerten ist mit einer Anzahl von 6863 Fällen ungefähr achtmal so groß wie die Gruppe mit erhöhten systolischen und/oder diastolischen Blutdruckwerten, die 825 Fälle umfasst (siehe Tabelle 6).

Anhand des t-Tests für unabhängige Stichproben sollte überprüft werden, ob die beiden oben beschriebenen Gruppen sich bezüglich des Alters, des Gewichts und des $B M I$ unterscheiden. Bezüglich der Altersverteilung zeigt sich zwischen der Gruppe mit normalen Blutdruckwerten und der Gruppe mit erhöhten systolischen und/oder diastolischen Blutdruckwerten ein kleiner aber höchst signifikanter Unterschied mit einem $p<0,001$. Der Mittelwert beträgt bei der Gruppe mit einem normalen Blutdruck 14,1 Jahre mit einer Standardabweichung von 1,99 und bei 
der Gruppe mit erhöhten systolischen und/oder diastolischen Blutdruckwerten 14,4 Jahre mit einer Standardabweichung von 1,92.

Für das Gewicht und den $B M I$ ergibt der t-Test mit $p<0,001$ ebenfalls ein höchst signifikantes Ergebnis: Die Gruppe der hypertensiven Jugendlichen weist mit einem Gewicht von 67,7 kg (Standardabweichung 19,0) und einem BMI von 23,7 (Standardabweichung 5,36) jeweils einen deutlich höheren Mittelwert auf als die Gruppe der normotensiven mit einem Gewicht von 56,8 kg (Standardabweichung 14,15) und einem BMI von 20,8 (Standardabweichung 3,76).

Anhand des Chi-Quadrat-Tests wurde überprüft, ob es zwischen beiden Gruppen einen signifikanten Unterschied bezüglich der Verteilung des Geschlechts und des sozialen Status gibt. Mit $p<0,001$ ist das Ergebnis der Geschlechtsverteilung höchst signifikant: Mit 61,3\% ist der Anteil der Jungen an der Gruppe mit hypertensiven systolischen und/oder diastolischen Blutdruckwerten höher als der Anteil der Mädchen mit 38,7\%. In der Gruppe mit einem normalen Blutdruckwert ist die Geschlechtsverteilung nahezu ausgeglichen. Bei dem sozialen Status wurde in die drei Statusgruppen niedriger, mittlerer und hoher Sozialstatus unterteilt. Der ChiQuadrat-Test zeigt keine signifikanten Unterschiede bezüglich der Verteilung des sozialen Status: Im Kollektiv der 11- bis 17-Jährigen weisen 27,4\% einen niedrigen, $47,2 \%$ einen mittleren und 25,3\% einen hohen Sozialstatus auf. Beim Gruppenvergleich zeigen $27,3 \%$ der normotensiven und $28,3 \%$ der hypertensiven Jugendlichen einen niedrigen Sozialstatus, 47,3\% im Vergleich zu 47,0\% einen mittleren und $25,4 \%$ im Vergleich zu $24,7 \%$ einen hohen Sozialstatus. 
Tab. 6 Allgemeine deskriptive Daten für das Gesamtkollektiv sowie für normotensive und hypertensive Jugendliche

\begin{tabular}{|c|c|c|c|c|}
\hline & $\begin{array}{l}\text { Gesamt- } \\
\text { kollektiv } \\
(\mathrm{N}=7688)\end{array}$ & $\begin{array}{l}\text { Normotensive } \\
\text { Jugendliche } \\
(\mathrm{N}=6863)\end{array}$ & $\begin{array}{l}\text { Hypertensive } \\
\text { Jugendliche } \\
(\mathrm{N}=825)\end{array}$ & $\begin{array}{l}\text { P } \\
\text { Werte }\end{array}$ \\
\hline Alter & $14.6 \pm 2.0$ & $14.6 \pm 2.0$ & $14.9 \pm 1.9$ & $<0.001$ \\
\hline männlich /Anzahl (\%) & 3951 (51.3) & 3441 (50.1) & 506 (61.3) & $<0.001$ \\
\hline Größe /cm & $164.7 \pm 11.3$ & $164.3 \pm 11.2$ & $168.0 \pm 11.5$ & $<0.001$ \\
\hline Gewicht /kg & $58.0 \pm 15.1$ & $56.8 \pm 14.2$ & $67.7 \pm 19.0$ & $<0.001$ \\
\hline$B M I /\left(\mathrm{kg} / \mathrm{m}^{2}\right)$ & $21.1 \pm 4.1$ & $20.8 \pm 3.8$ & $23.7 \pm 5.4$ & $<0.001$ \\
\hline Systolischer BD /mmHg & $114.7 \pm 10.9$ & $112.5 \pm 8.8$ & $133.6 \pm 8.2$ & $<0.001$ \\
\hline Diastolischer BD /mmHg & $68.3 \pm 7.6$ & $67.1 \pm 6.7$ & $77.8 \pm 7.5$ & $<0.001$ \\
\hline $\mathrm{HF} / \mathrm{min}^{-1}$ & $75.8 \pm 11.7$ & $75.2 \pm 11.4$ & $81.1 \pm 13.5$ & $<0.001$ \\
\hline $\begin{array}{l}\text { Niedriger Sozialstatus } \\
(\%)\end{array}$ & 27.4 & 27.3 & 28.2 & 0.578 \\
\hline
\end{tabular}




\subsection{Korrelation zwischen Subskalen und übergreifendem Mess- wert sowie zwischen Eigen- und Elternangaben bei Lebens- qualität und psychosozialen Problemen}

Es sollte die Korrelation zwischen den Eigen- und den Elternangaben bezüglich der Lebensqualität (KINDL-R) sowie der psychosozialen Schwierigkeiten (SDQ) überprüft werden. Da sowohl die KINDL-R- als auch die SDQ-Angaben ein ordinales Skalenniveau aufweisen, wurde der Rangkorrelationskoeffizienten nach Spearman (Spearman-Rho) verwendet, der von einer Gleichabständigkeit zwischen den Skalenwerten bzw. Rängen ausgeht (Fahrmeir et al. 2004). Die Signifikanz wurde zum Niveau von $\alpha=5 \%$ getestet.

Die Lebensqualität wurde bei den Eigenangaben und bei den Elternangaben anhand des KINDL-R-Fragebogens erfasst. Bei den Elternangaben zeigen die Subskalen der Lebensqualität mit dem Globalwert einen minimalen Korrelationskoeffizienten (Rho) von 0,606 in der Subskala „Freunde“ und ein maximales Rho von 0,754 in der Subskala „psychisches Wohlbefinden“.

Bei den Eigenangaben der Kinder und Jugendlichen weist die Subskala „Familie“ mit einem Wert von 0,596 ein minimales Rho mit dem übergreifenden Messwert auf und die Subskala „psychisches Wohlbefinden“ mit einem Wert von 0,654 ein maximales Rho.

Die psychosozialen Probleme und Stärken wurden anhand des Strength and Difficulties Questionnaire (SDQ) erfasst. Bei den Elternangaben zeigt der Bereich „Probleme mit Gleichaltrigen“ mit dem Gesamtproblemwert ein minimales Rho von 0,591 und der Bereich „Hyperaktivität“ ein maximales Rho von 0,753. Auch bei den Eigenangaben weist der Bereich „Probleme mit Gleichaltrigen“ mit dem Gesamtproblemwert ein minimales Rho von 0,526 auf, der Bereich „Hyperaktivität“ zeigt dagegen ein maximales Rho von 0,696.

Sämtliche oben genannten Rangkorrelationskoeffizienten nach Spearman sind mit $\mathrm{p}<0,001$ höchst signifikant. Sowohl zwischen den Subskalen des KINDL-R und dem übergreifenden Messwert als auch zwischen den einzelnen Bereichen des $S D Q$ und dem Gesamtproblemwert zeigt sich eine mittlere bis hohe Korrelation. 
Die Subskalen des KINDL-R-Fragebogens zeigen zwischen den Eltern- und den Eigenangaben ein minimales Rho von 0,264 in der Subskala „Selbstwert“ und ein maximales Rho von 0,465 bei dem übergreifenden Messwert der Lebensqualität (siehe Tabelle 7).

Tab. 7 Korrelationen (Spearman's Rho) zwischen Eigen- und Elternangaben hinsichtlich der Lebensqualität (KINDL-R)

\begin{tabular}{|c|c|c|}
\hline KINDL-R & Rho & Signifikanz \\
\hline $\begin{array}{c}\text { Körperliches Wohl- } \\
\text { befinden }\end{array}$ & 0,435 & $\mathrm{p} \leq 0,001$ \\
\hline $\begin{array}{c}\text { Psychisches Wohl- } \\
\text { befinden }\end{array}$ & 0,293 & $\mathrm{p} \leq 0,001$ \\
\hline Selbstwert & 0,264 & $\mathrm{p} \leq 0,001$ \\
\hline Familie & 0,424 & $\mathrm{p} \leq 0,001$ \\
\hline Freunde & 0,367 & $\mathrm{p} \leq 0,001$ \\
\hline Schule & 0,459 & $\mathrm{p} \leq 0,001$ \\
\hline
\end{tabular}

Zwischen den Eltern- und den Eigenangaben des SDQ zeigt der Stärkenbereich „Prosoziales Verhalten“ ein minimales Rho von 0,304 und der Gesamtproblemwert ein maximales Rho von 0,444 (siehe Tabelle 8).

Tab. 8 Korrelationen (Spearman's Rho) zwischen Eigen- und Elternangaben hinsichtlich psychosozialer Probleme und Stärken

\begin{tabular}{|c|c|c|}
\hline SDQ & Rho & Signifikanz \\
\hline Emotionale Probleme & 0,360 & $\mathrm{p} \leq 0,001$ \\
\hline Verhaltensprobleme & 0,331 & $\mathrm{p} \leq 0,001$ \\
\hline Hyperaktivität & 0,425 & $\mathrm{p} \leq 0,001$ \\
\hline Peer-Probleme & 0,374 & $\mathrm{p} \leq 0,001$ \\
\hline Prosoziales Verhalten & 0,304 & $\mathrm{p} \leq 0,001$ \\
\hline Gesamtproblemwert & 0,444 & $\mathrm{p} \leq 0,001$ \\
\hline
\end{tabular}


Die genannten Rangkorrelationskoeffizienten nach Spearman zwischen Elternund Eigenangaben sind mit $p \leq 0,001$ höchst signifikant. Insgesamt zeigen sich aber sowohl im KINDL-R als auch im SDQ eher schwache Korrelationen zwischen Selbst- und Fremdeinschätzung.

\section{3 Überprüfung der Verteilung von Lebensqualität, psychoso- zialen Problemen und Schutzfaktoren bei normotensiven und hypertensiven Jugendlichen}

Ziel der Testung war es herauszufinden, ob es zwischen der Gruppe der Kinder und Jugendlichen mit normalen Blutdruckwerten und der Gruppe mit erhöhten systolischen und/oder diastolischen Blutdruckwerten Unterschiede in den Bereichen Lebensqualität, psychosoziale Probleme und Stärken sowie Ressourcen und Schutzfaktoren gibt. Zum nichtparametrischen Vergleich der zwei unabhängigen Stichproben wurde der U-Test nach Mann und Whitney verwendet. Die Testung fand zum Signifikanzniveau von $\alpha=5 \%$ statt.

Die Lebensqualität wurde anhand des KINDL-R-Fragebogens bestimmt, wobei die Beurteilung der Verteilung zunächst anhand der Eigenangaben durchgeführt wurden. Die Skalierung erfolgte durch Werte von 0 bis 100, wobei höhere Werte mit einer besseren Lebensqualität einhergehen. 


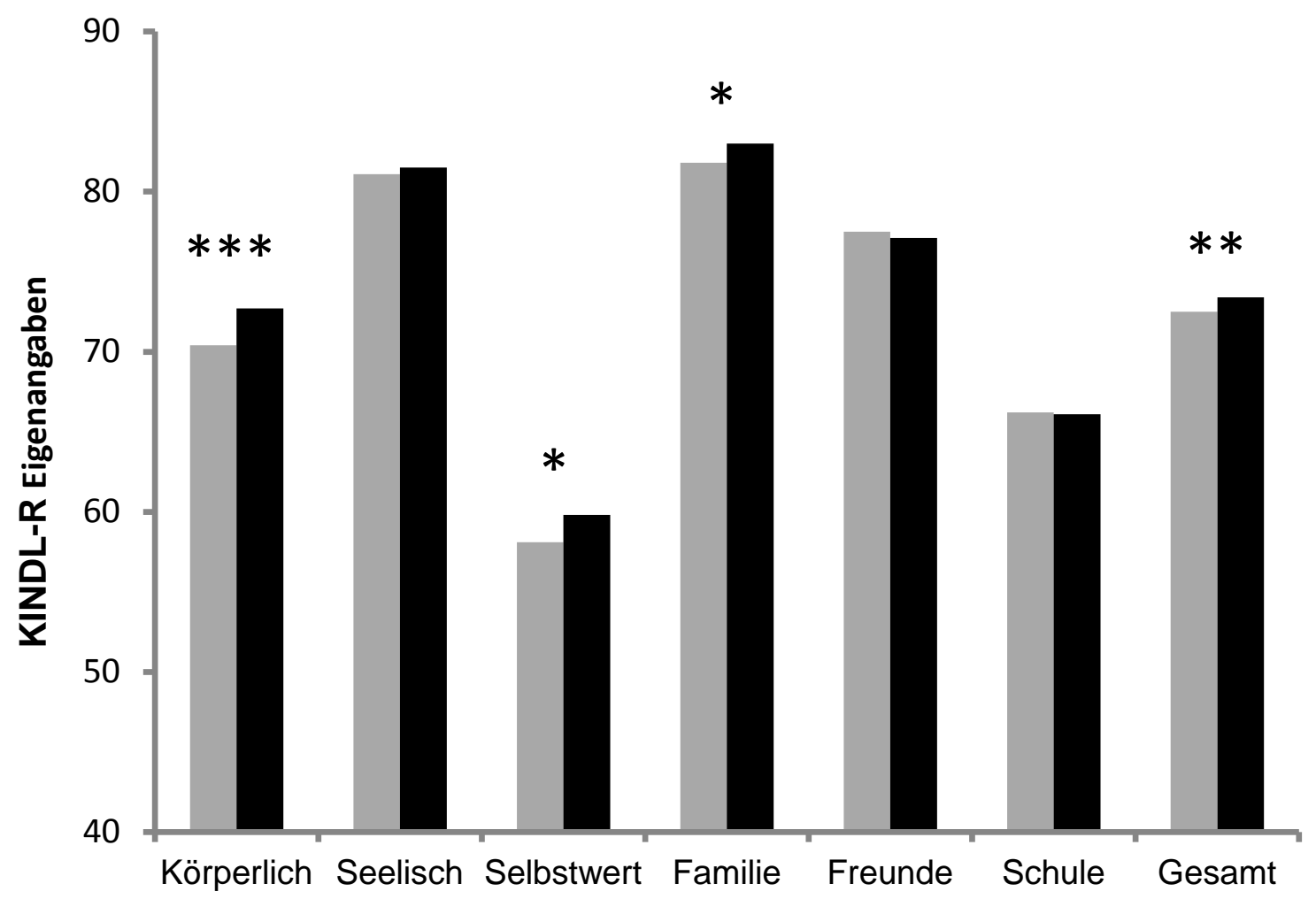

\begin{abstract}
Abb. 4 Überblick über die Unterschiede zwischen hypertensiven und normotensiven Jugendlichen in den einzeInen Bereichen der Lebensqualität anhand der Eigenangaben
\end{abstract}

Hypertensive Jugendliche (schwarz) und normotensive Jugendliche (grau) unterscheiden sich in den gekennzeichneten Bereichen der Lebensqualität. Dargestellt sind die Mittelwerte für jede aufgeführte Subskala sowie für den übergreifenden Messwert. Signifikante Unterschiede werden durch Sternchen gekennzeichnet ( ${ }^{\star} p<0.05$, ${ }^{\star \star} p<0.01,{ }^{\star \star \star} p<0.001$ ).

Zwischen den hypertensiven und normotensiven Jugendlichen (Abbildung 4) zeigen sich mit $p<0,05$ signifikante Unterschiede in den Bereichen "Selbstwert" $(69,1 \pm 15,0$ vs. $67,1 \pm 15,2)$ und „Familie“ $(78,0 \pm 15,3$ vs. $76,2 \pm 15,1)$. Mit einer Irrtumswahrscheinlichkeit von $\mathrm{p}<0,001$ sind die Unterschiede im Bereich des körperlichen Wohlbefindens (75,3 $\pm 18,1$ vs. $74,0 \pm 17,3)$ höchst sigifikant.

Der übergreifende Messwert unterscheidet sich mit $p<0,01$ sehr signifikant bei beiden Gruppen $(75,2 \pm 10,3$ vs. $74,1 \pm 10,3)$. In allen genannten Bereichen der Lebensqualität weist jeweils die Gruppe der Hypertensiven höhere Skalenwerte für die Subskalen auf. 
Die anderen Subskalen „psychisches Wohlbefinden“, „Freunde“ und „Schule“ erreichen nicht das Signifikanzniveau.

Im Anschluss erfolgte die Überprüfung der Verteilung der psychosozialen Probleme und Stärken bei hypertensiven und normotensiven Jugendlichen zunächst anhand der Eigenangaben der Kinder und Jugendlichen.

Die psychosozialen Schwierigkeiten und Stärken wurden anhand des Strength and Difficulties Questionnaire (SDQ) bestimmt. Ein höherer Skalenwert geht dabei mit größeren psychosozialen Problemen einher.

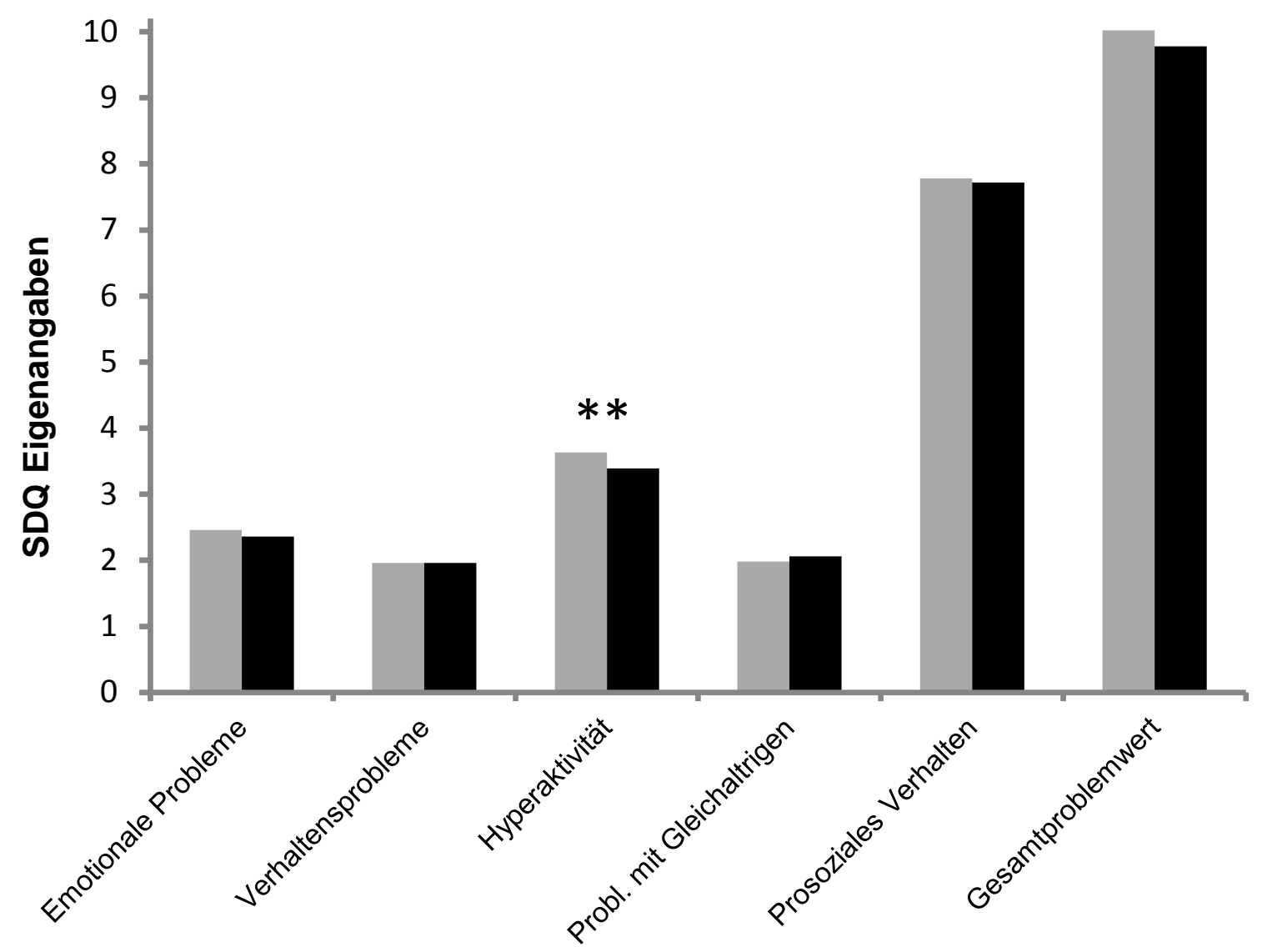

Abb. 5 Überblick über die Unterschiede zwischen normotensiven und hypertensiven Jugendlichen in den Bereichen der psychosozialen Schwierigkeiten und Stärken anhand der Eigenangaben

Hypertensive (schwarz) und normotensive (grau) Jugendliche unterscheiden sich in den gekennzeichneten Bereichen des SDQ. Dargestellt sind die Mittelwerte für jede aufgeführte Subskala sowie für den Gesamtproblemwert. Signifikante Unterschiede werden durch Sternchen gekennzeichnet $\left({ }^{*} p<0.05,{ }^{* *} p<0.01,{ }^{* \star *} p<0.001\right)$. 
Zwischen der Gruppe der Normotensiven und der Gruppe der Hypertensiven zeigt sich mit einem $p<0,01$ ein sehr signifikanter Unterschied in dem Bereich der Hyperaktivität (Abbildung 5). Die Jugendlichen mit erhöhten arteriellen Blutdruckwerten weisen mit 3,39 $\pm 2,02$ einen etwas geringeren Mittelwert auf als die Normotensiven mit 3,63 $\pm 2,02$.

Die anderen Bereiche des SDQ wie „emotionale Probleme", „Verhaltensprobleme", „Probleme mit Gleichaltrigen", „prosoziales Verhalten" und der Gesamtproblemwert zeigen keine signifikanten Unterschiede zwischen den Gruppen.

Weiterhin wurde die Verteilung der Schutzfaktoren bei hypertensiven und normotensiven Jugendlichen ebenfalls anhand der Eigenangaben überprüft:

Bei den Risiko- und Schutzfaktoren wurden die Bereiche der sozialen Unterstützung sowie die personalen und familiären Ressourcen betrachtet (siehe Tabelle 9). In allen drei Bereichen zeigen sich keine signifikanten Unterschiede zwischen der Gruppe mit normalen Blutdruckwerten und der Gruppe mit systolischen und/oder diastolischen Blutdruckwerten oberhalb des 95. Perzentils.

Tab. 9 Risiko- und Schutzfaktoren mit Angabe der mittleren Ränge von normotensiven und hypertensiven Jugendlichen sowie dem entsprechenden Signifikanzniveau

\begin{tabular}{cccc}
\hline & $\begin{array}{c}\text { Mittlerer Rang Hyper- } \\
\text { tensive Jugendliche }\end{array}$ & $\begin{array}{c}\text { Mittlerer Rang Nor- } \\
\text { motensive Jugendi- } \\
\text { che }\end{array}$ & $\begin{array}{c}\text { Signifi- } \\
\text { kanz }\end{array}$ \\
\hline $\begin{array}{c}\text { Soziale Unterstützung } \\
\text { (Eigenangaben) }\end{array}$ & 3541 & 3661 & 0,132 \\
Personale Ressourcen & 3708 & 3652 & 0,477 \\
$\quad \begin{array}{c}\text { (Eigenangaben) } \\
\text { Familiäre Ressourcen }\end{array}$ & 3648 & 3657 & 0,907 \\
(Eigenangaben) & 3451 & 3447 & 0,951 \\
\hline $\begin{array}{c}\text { Familiäre Ressourcen } \\
\text { (Elternangaben) }\end{array}$ & & & \\
\hline
\end{tabular}


Bei der ergänzenden Überprüfung der Lebensqualität anhand der Elternangaben zeigen die beiden Gruppen mit $p<0,01$ einen sehr signifikanten Unterschied im Bereich des körperlichen Wohlbefindens (75,3 \pm 18,1 vs.74,0 $\pm 17,3)$, des Selbstwerts $(69,1 \pm 15,0$ vs.67,1 $\pm 15,2)$ und im übergreifenden Messwert $(75,2 \pm$ $10,3$ vs. $74,1 \pm 10,3)$. Dabei weist die Gruppe der hypertensiven Jugendlichen jeweils einen höheren Skalenwert und damit eine bessere Lebensqualität auf (Abbildung 8).

Der Bereich „Familie" zeigt sich mit $p<0,001$ als höchst signifikant unterschiedlich zwischen den Gruppen: Die Hypertensiven haben mit 78,0 \pm 15,3 einen etwas höheren Mittelwert und damit eine bessere Lebensqualität als die Normotensiven mit $76,2 \pm 15,1$.

Die Bereiche „Psychisches Wohlbefinden“, „Freunde“ und „Schule“ erweisen sich als nicht signifikant. 


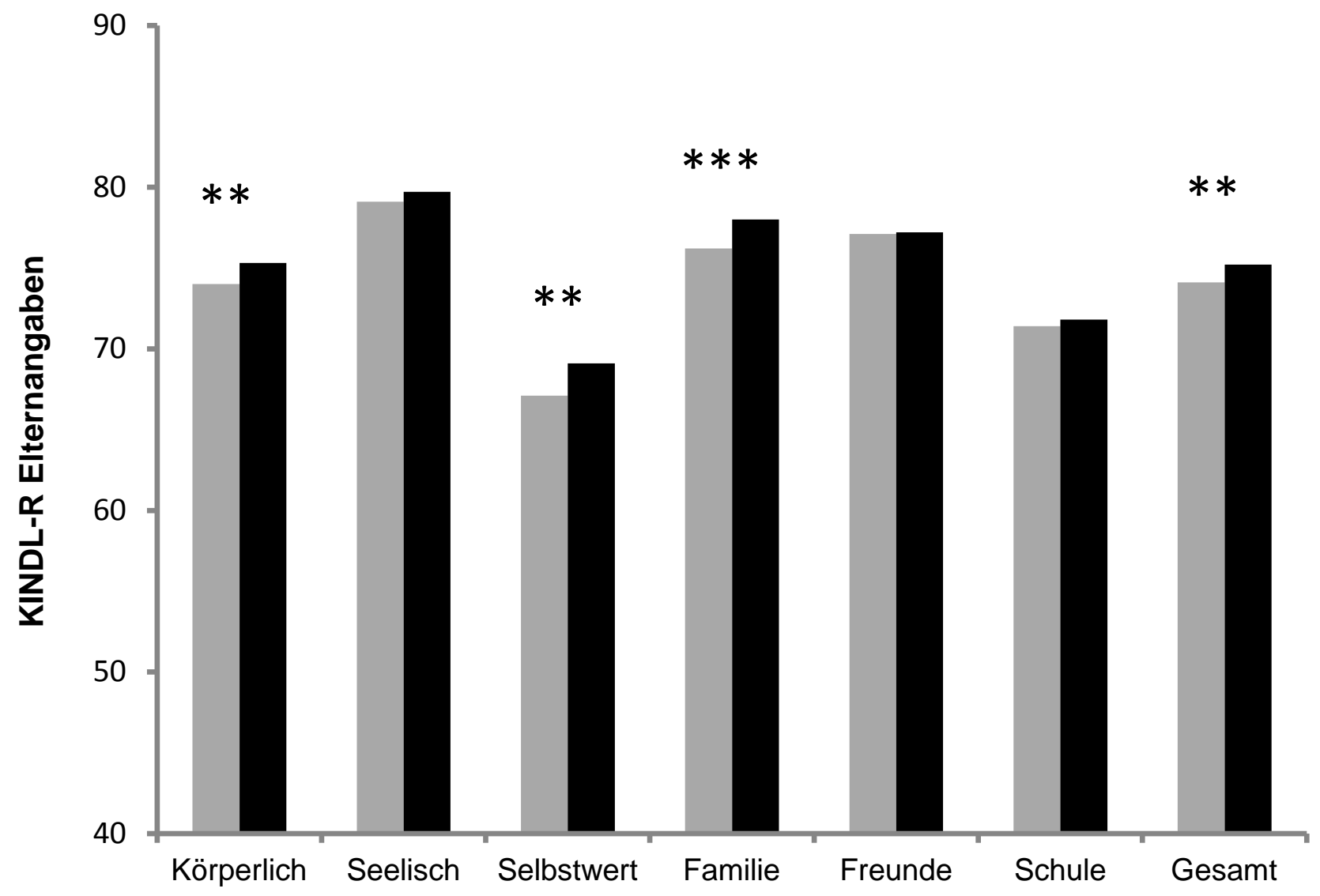

Abb. 6 Überblick über die Unterschiede zwischen hypertensiven und normotensiven Jugendlichen in den einzeInen Bereichen der Lebensqualität anhand der Elternangaben

Hypertensive (schwarz) und normotensive (grau) Jugendliche unterscheiden sich in den gekennzeichneten Bereichen der Lebensqualität. Dargestellt sind die Mittelwerte für jede aufgeführte Subskala sowie für den übergreifenden Messwert. Signifikante Unterschiede werden durch Sternchen gekennzeichnet $\left({ }^{\star} p<0.05,{ }^{* \star} p<0.01,{ }^{* * *} p<0.001\right)$.

Außerdem wurden die hypertensiven und normotensiven Jugendlichen bezüglich möglicher Unterschiede in der Verteilung von psychosozialen Probleme und Stärken anhand der Elternangaben überprüft:

Auch hier wurden die psychosozialen Schwierigkeiten und Stärken anhand des SDQ bestimmt. 


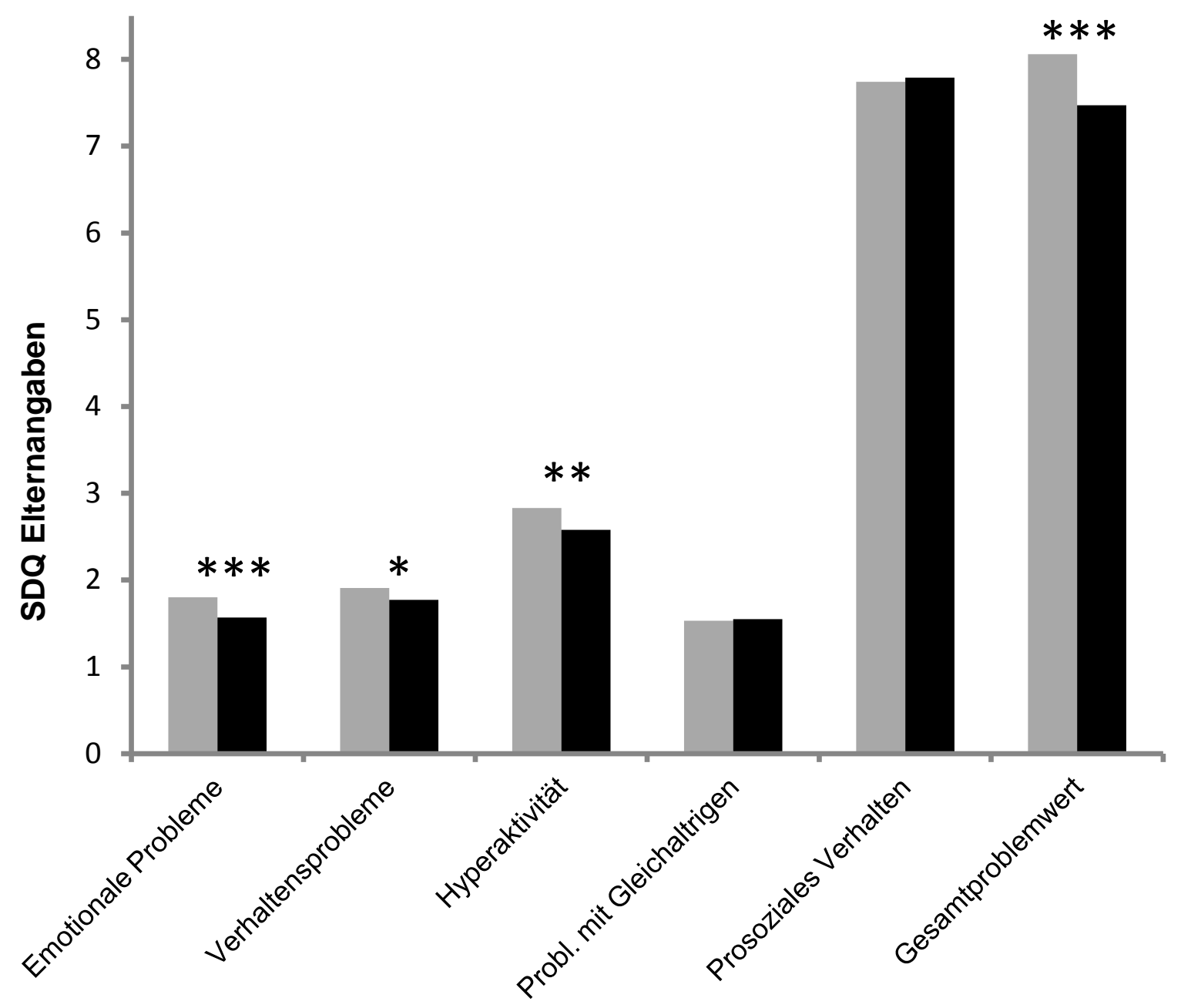

Abb. 7 Überblick über die Unterschiede zwischen normotensiven und hypertensiven Jugendlichen in den Bereichen der psychosozialen Probleme und Stärken anhand der Elternangaben

Hypertensive (schwarz) und normotensive (grau) Jugendliche unterscheiden sich in den gekennzeichneten Bereichen des SDQ. Dargestellt sind die Mittelwerte für jede aufgeführte Subskala sowie für den Gesamtproblemwert. Signifikante Unterschiede werden durch Sternchen gekennzeichnet $\left({ }^{*} p<0.05,{ }^{* \star} p<0.01,{ }^{* * *} p<0.001\right)$.

Mit einer Irrtumswahrscheinlichkeit von $p \leq 0,001$ weisen die Jugendlichen mit erhöhten arteriellen Blutdruckwerten im Vergleich zu den normotensiven Jugendlichen in der Subskala „emotionale Probleme" (1,57 $\pm 1,80$ vs. 1,80 $\pm 1,87)$ und bei dem Gesamtproblemwert $(7,47 \pm 5,17$ vs. $8,06 \pm 5,16)$ höchst signifikante Unterschiede auf (Abbildung 9). 
Der Bereich „Hyperaktivität" $(2,58 \pm 2,10$ vs. $2,83 \pm 2,20)$ unterscheidet sich mit $p<0,01$ sehr signifikant und die Subskala „Verhaltensprobleme" mit $p<0,05$ signifikant $(1,77 \pm 1,53$ vs. $1,91 \pm 1,55)$.

Die Bereiche „Probleme mit Gleichaltrigen“ und „Prosoziales Verhalten“ sind nicht signifikant unterschiedlich zwischen den Gruppen.

Bezüglich der Risiko- und Schutzfaktoren wurden anhand der Elternangaben nur die familiären Ressourcen untersucht. Zwischen den beiden Gruppen zeigt sich jedoch kein signifikanter Unterschied (siehe Tabelle 9, S.43).

\section{4 Überprüfung von Lebensqualität sowie von psychosozialen Problemen und Stärken im Modell mit möglichen Confoundern}

Anhand der binären logistischen Regression sollten die Variablen, die sich bei der univariaten Testung im Mann-Whitney-U-Test als signifikant unterschiedlich zwischen den beiden Gruppen erwiesen, im multivariaten Modell mit Kovariablen zum Signifikanzniveau von $\alpha=5 \%$ getestet werden. Für den besseren Vergleich der Stärke und der Art des Zusammenhangs zwischen erhöhten systolischen und/oder diastolischen Blutdruckwerten und den verschiedenen Variablen wurden die Odds Ratios im univariaten sowie im multivariaten Modell betrachtet.

Im Modell wurden die Variablen „Alter“, „Geschlecht“, „BMl“ und „Sozialer Status“ als Kontrollvariablen eingesetzt.

\subsubsection{Univariate Überprüfung der relevanten Variablen}

Bei der univariaten Testung wurden die Variablen der Lebensqualität (KINDL-R) und der psychosozialen Probleme und Stärken (SDQ) jeweils als einziges Differenzierungsmerkmal im Modell mit dem erhöten Blutdruck als abhängige Variable betrachtet. Die genauen Odds Ratios zu den einzelnen Variablen des KINDL-R und SDQ sind den Tabellen 10 und 11 (S. 49) zu entnehmen. Es soll ein 
besserer Vergleich mit den Ergebnissen der multivariaten Testung ermöglicht werden.

\subsubsection{Multivariate Testung im Modell 1}

Bei der multivariaten Testung wurden die Variablen der Lebensqualität sowie der psychosozialen Probleme und Stärken jeweils einzeln mit den Kovariablen „Alter“, „Geschlecht“, „BMI“ und „sozialem Status“ als Prädiktoren des Blutdrucks im Modell 1 überprüft.

Bei den Elternangaben (Tabelle 10) zeigen sich bei der Lebensqualität in den Bereichen „Selbstwert", „Familie“ und dem übergreifenden Messwert mit $p<0,001$ höchst signifikante OR>1. Die Subskala „körperliches Wohlbefinden“ weist mit $p<0,01$ ein sehr signifikantes $O R>1$. Bei den Elternangaben $z u$ den psychosozialen Problemen und Stärken (Tabelle 10) ergeben sich für die Subskalen „Verhaltensauffälligkeiten“ und „Hyperaktivität“ sowie den Gesamtproblemwert mit $p<0,001$ jeweils höchst signifikante $O R<1$. Der Bereich „emotionale Probleme“ weist mit $p<0,01$ ein sehr signifikantes $O R<1$ auf.

Bei den Eigenangaben zur Lebensqualität (Tabelle 11) weisen die Subskala „körperliches Wohlbefinden“ sowie der übergreifende Messwert der Lebensqualität mit $p<0,001$ höchst signifikante OR $>1$ auf. Der Bereich „Familie“ zeigt mit $p<0,01$ ein sehr signifikantes $O R>1$ und der Bereich "Selbstwert" mit $p<0,05$ ein signifikantes $\mathrm{OR}>1$. Bei den Eigenangaben zu psychosozialen Problemen und Stärken (Tabelle 11) lässt sich in der Subskala „Hyperaktivität“ mit einem $p<0,001$ ein höchst signifikantes $\mathrm{OR}<1$ feststellen. Wie bei der univariaten Analyse zeigt sich ebenfalls bei der multivariaten Testung im Modell 1, dass ein ansteigender Skalenwert in der Lebensqualität mit einem erhöhten Risiko und ein ansteigender Skalenwert bei den psychosozialen Schwierigkeiten mit einem geringeren Risiko für hypertensive systolische und/oder diastolische Blutdruckwerte einhergeht. 
Tab. 10 Erhöhter arterieller Blutdruck als abhängige Variable im Modell mit den KINDL-R- ISDQ-Variablen (aus den Elternangaben)

\begin{tabular}{|c|c|c|c|c|}
\hline Variable & univariat & multivariat & Signifikanz \\
Elternangaben & OR & $\begin{array}{l}\text { OR } \\
\text { OR } 1\end{array}$ & Signifikanz \\
\hline $\begin{array}{c}\text { körperliches Wohlbe- } \\
\text { finden }\end{array}$ & 1,005 & $\mathrm{p}<0,05$ & 1,007 & $\mathrm{p}<0,01$ \\
\hline Selbstwert & 1,009 & $\mathrm{p} \leq 0,001$ & 1,011 & $\mathrm{p}<0,001$ \\
\hline Familie & 1,008 & $\mathrm{p}<0,01$ & 1,010 & $\mathrm{p}<0,001$ \\
\hline $\begin{array}{c}\text { übergreifender Mess- } \\
\text { wert (KINDL-R) }\end{array}$ & 1,010 & $\mathrm{p}<0,01$ & 1,016 & $\mathrm{p}<0,001$ \\
\hline emotionale Probleme & 0,931 & $\mathrm{p} \leq 0,001$ & 0,936 & $\mathrm{p}<0,01$ \\
\hline $\begin{array}{c}\text { Verhaltensauffälligkei- } \\
\text { ten }\end{array}$ & 0,942 & $\mathrm{p}<0,05$ & 0,893 & $\mathrm{p}<0,001$ \\
\hline Hyperaktivität & 0,948 & $\mathrm{p}<0,01$ & 0,910 & $\mathrm{p}<0,001$ \\
\hline $\begin{array}{c}\text { Gesamtproblemwert } \\
\text { (SDQ) }\end{array}$ & 0,977 & $\mathrm{p}<0,01$ & 0,958 & $\mathrm{p}<0,001$ \\
\hline
\end{tabular}

Modell $1=\quad$ Testung der KINDL-R und SDQ Variablen (Elternangaben) einzeln im Modell mit Alter, Geschlecht, sozialem Status, BMI; Methode= Einschluss

Tab. 11 Erhöhter arterieller Blutdruck als abhängige Variable im Modell mit den KINDL-R- I SDQ-Variablen (aus den Eigenangaben)

\begin{tabular}{|c|c|c|c|c|}
\hline Variable & univariat & & $\begin{array}{l}\text { multivariat: } \\
\text { Eodell 1 } \\
\text { OR }\end{array}$ & Signifikanz \\
\hline $\begin{array}{c}\text { körperliches Wohlbe- } \\
\text { finden }\end{array}$ & 1,009 & $\mathrm{p}<0,001$ & 1,012 & $\mathrm{p}<0,001$ \\
\hline Selbstwert & 1,005 & $\mathrm{p}<0,05$ & 1,005 & $\mathrm{p}<0,05$ \\
\hline Familie & 1,005 & $\mathrm{p}<0,05$ & 1,007 & $\mathrm{p}<0,01$ \\
\hline Total/KINDL-R & 1,008 & $\mathrm{p}<0,05$ & 1,014 & $\mathrm{p} \leq 0,001$ \\
\hline Hyperaktivität & 0,944 & $\mathrm{p}<0,01$ & 0,934 & $\mathrm{p} \leq 0,001$ \\
\hline
\end{tabular}

Modell $1=\quad$ Testung der KINDL-R und SDQ Variablen (Eigenangaben) einzeln im Modell mit Alter, Geschlecht, sozialem Status, BMI; Methode= Einschluss 
Die exakten $O R$ und Signifikanzangaben der Kontrollvariablen „Alter", „Geschlecht", „BMI" und „Sozialer Status“ im Modell 1 mit den einzelnen Variablen der Lebensqualität und der psychischen Gesundheit sind den Tabellen I bis XIII im Anhang 6 zu entnehmen.

Exemplarisch werden nun die $O R$ mit Signifikanzangaben der Kontrollvariablen am Beispiel des übergreifenden Messwerts und des Gesamtproblemwerts der Elternangaben erläutert:

Das Geschlecht und der $B M I$ zeigen im Modell 1 mit $p<0,001$ jeweils ein höchst signifikantes Odds Ratio. Ein ansteigender BMI geht mit einem größeren Risiko für einen erhöhten arteriellen Blutdruck einher $(O R>1)$. Männliche Jugendliche zeigen auch in der multivariaten Analyse im Modell 1 ein größeres Risiko für erhöhte arterielle Blutdruckwerte als weibliche Jugendliche $(O R>1)$. Bei dem sozialen Status erweist sich das Odds Ratio der Ausprägung "niedriger" versus „hoher sozialer Status" mit $\mathrm{p}<0,05$ nur im Modell mit dem übergreifenden Messwert der Lebensqualität als signifikant. Im Vergleich zu einem hohen Sozialstatus führt ein niedriger mit einem $O R<1$ zu einem geringeren Risiko für einen erhöhten arteriellen Blutdruck. Das Alter erweist sich in der mulitvariaten Testung sowohl im Modell mit dem Gesamtproblemwert als auch mit dem übergreifenden Messwert der Lebensqualität als nicht mehr signifikant. In Tabelle 12 und 13 sind die genauen Odds Ratios mit Signifikanzniveau für die Kontrollvariablen im Modell mit dem übergreifenden Messwert der Lebensqualität bzw. dem Gesamtproblemwert aufgeführt. 
Tab. 12 OR und Signifikanz des übergreifenden Messwerts der Lebensqualität (Elternangaben) sowie der Kontrollvariablen im Modell 1

\begin{tabular}{|c|c|c|}
\hline $\begin{array}{c}\text { Lebensqualität } \\
\text { (Elternangaben) }\end{array}$ & OR & Signifikanz \\
\hline übergreifender Messwert & 1,016 & $p<0,001$ \\
\hline Alter & 1,000 & $\varnothing$ sign. \\
\hline Geschlecht & 1,619 & $p<0,001$ \\
\hline BMI & 1,165 & $p<0,001$ \\
\hline Sozialer Status & & $\emptyset$ sign. \\
\hline "mittel" vs. „hoch" & 0,910 & $p<0,05$. \\
\hline "niedrig" vs. "hoch" & 0,789 & \multicolumn{2}{c|}{} \\
\hline
\end{tabular}

Tab. 13 OR und Signifikanz des Gesamtproblemwerts der psychischen Gesundheit (Elternangaben) sowie der Kontrollvariablen im Modell 1

\begin{tabular}{|c|c|c|}
\hline $\begin{array}{c}\text { Psychische Gesundheit } \\
\text { (Elternangaben) }\end{array}$ & OR & Signifikanz \\
\hline Gesamtproblemwert & 0,958 & $p<0,001$ \\
\hline Alter & 0,989 & $\varnothing$ sign. \\
\hline Geschlecht & 1,772 & $p<0,001$ \\
\hline BMI & 1,169 & $p<0,001$ \\
\hline Sozialer Status & & $\varnothing$ sign. \\
\hline "mittel" vs. „hoch" & 0,953 & $\varnothing$ sign. \\
\hline "niedrig vs. „hoch" & 0,878 & \\
\hline
\end{tabular}




\subsection{Explorative Überprüfung der normotensiven und hyperten- siven Jugendlichen auf weitere Unterschiede}

\subsubsection{Chi-Quadrat-Test zur explorativen Überprüfung der beiden Gruppen auf weitere Unterschiede}

Mit Hilfe des Chi-Quadrat Tests sollte auf weitere Unterschiede zwischen der Gruppe der Hypertensiven und der Gruppe der Normotensiven geprüft werden.

Dazu wurden verschiedene Aspekte zur Gesundheit, zum Freizeitverhalten und zum allgemeinen Befinden betrachtet.

Im Bereich "Gesundheit" (siehe Tabelle 14) zeigen sich weder beim subjektiven Gesundheitsstatus noch bei der Angabe von weiteren Erkrankungen, bei der Inanspruchnahme bzw. dem Bedarf von medizinischer und nichtmedizinischer Versorgung oder bei der Medikamenteneinnahme signifikante Unterschiede. Nur in der Angabe zu einem ärztlich oder psychologisch diagnostizierten ADHS unterscheiden sich die Gruppen signifikant: Bei Jugendlichen mit erhöhten arteriellen Blutdruckwerten wurde mit 3,8\% seltener als bei denjenigen mit einem normalen Blutdruck mit 5,8\% ein Hyperaktivitätssyndrom diagnostiziert. 
Tab. 14 Verteilung von Gesundheitsmerkmalen bei hypertensiven und normotensiven Jugendlichen und Signifikanzangabe des Chi-Quadrat-Tests

\begin{tabular}{|c|c|c|c|c|}
\hline & $\begin{array}{l}\text { Gesamt- } \\
\text { kollektiv }\end{array}$ & $\begin{array}{l}\text { Hypertensive } \\
\text { Jugendliche }\end{array}$ & $\begin{array}{l}\text { Normotensive } \\
\text { Jugendliche }\end{array}$ & $\begin{array}{c}\text { Signifi- } \\
\text { kanz }\end{array}$ \\
\hline $\begin{array}{l}\text { Sehr schlech- } \\
\text { ter/schlechter subjektiver } \\
\text { Gesundheitsstatus }\end{array}$ & $\begin{array}{l}0,6 \% \\
N=37\end{array}$ & $\begin{array}{l}1,2 \% \\
N=8\end{array}$ & $\begin{array}{l}0,5 \% \\
N=29\end{array}$ & 0,060 \\
\hline Erkrankungen & $\begin{array}{l}28,1 \% \\
N=1963\end{array}$ & $\begin{array}{l}27,7 \% \\
N=207\end{array}$ & $\begin{array}{l}28,1 \% \\
N=1752\end{array}$ & 0,831 \\
\hline $\begin{array}{c}\text { Verstärkte Inanspruch- } \\
\text { nahme medizinischer } \\
\text { Versorgung }\end{array}$ & $\begin{array}{l}6,9 \% \\
N=495\end{array}$ & $\begin{array}{l}6,2 \% \\
N=47\end{array}$ & $\begin{array}{l}7,0 \% \\
N=444\end{array}$ & 0,444 \\
\hline Medikamenteneinnahme & $\begin{array}{l}15,8 \% \\
N=1126\end{array}$ & $\begin{array}{l}14,9 \% \\
N=113\end{array}$ & $\begin{array}{l}15,8 \% \\
N=1009\end{array}$ & 0,530 \\
\hline Diagnose ADHS & $\begin{array}{l}6,2 \% \\
N=430\end{array}$ & $\begin{array}{l}4,2 \% \\
N=31\end{array}$ & $\begin{array}{l}6,4 \% \\
N=396\end{array}$ & 0,021 \\
\hline
\end{tabular}

Beim Freizeitverhalten zeigt sich ein Unterschied hinsichtlich des Mediengebrauchs: Höchst signifikante Unterschiede bestehen zwischen der Gruppe der Normotensiven und der Gruppe der Hypertensiven mit einer Irrtumswahrscheinlichkeit von $p \leq 0,001$ bei der Nutzung von TV und Video: Maximal eine halbe Stunde pro Tag sehen $26,2 \%$ der normotensiven im Vergleich zu 19,5\% der hypertensiven Jugendlichen fern. 1-2 Stunden pro Tag schauen $51,3 \%$ der normotensiven und $55,0 \%$ der hypertensiven Jugendlichen. Eine Nutzung des Fernsehers über drei Stunden täglich ist bei $22,5 \%$ der Normotensiven und bei $25,4 \%$ der Hypertensiven zu verzeichnen. 


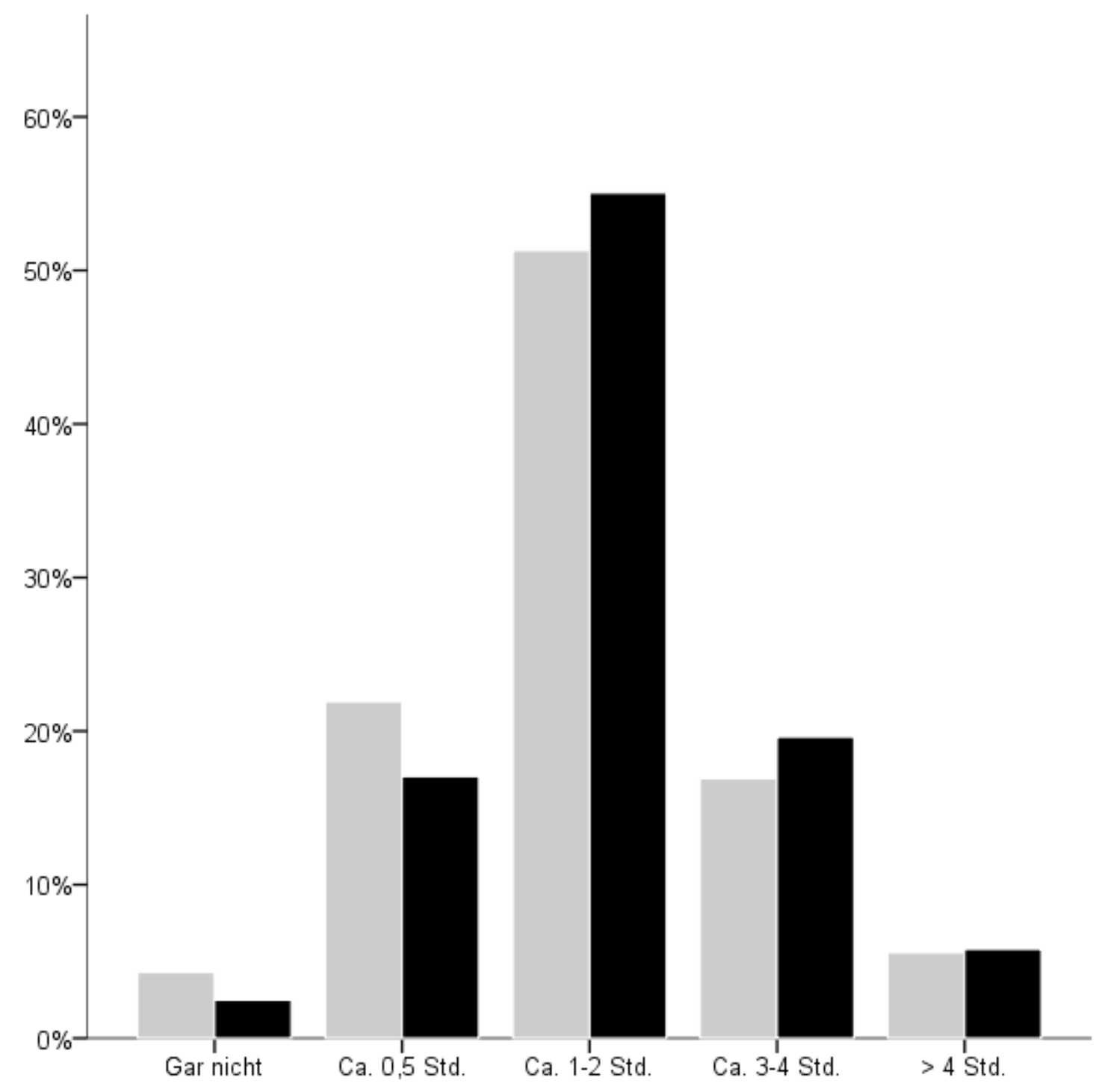

Abb. 8 Verteilung der Nutzung des Fernsehers (in Stunden pro Tag)

Darstellung des Gebrauchs des Fernsehers in Prozent, jeweils getrennt für hypertensive (schwarz) und normotensive (grau) Jugendliche.

Mit $p<0,001$ gibt es auch einen sehr signifikanten Unterschied bei dem Gebrauch von Computern: 1-2 Stunden pro Tag nutzen mit 28,9\% im Vergleich zu 28,4\% bei den hypertensiven Jugendlichen beide Gruppen ungefähr gleich häufig den Computer. Über drei Stunden wird der Computer von 11,3\% der normotensiven und von 15,7\% der Jugendlichen mit erhöhtem arteriellen Blutdruck verwendet. Bei der Gruppe mit erhöhten systolischen und/oder diastolischen Blutdruckwerten ist also jeweils ein längerer Gebrauch von TV und Computer zu verzeichnen. 


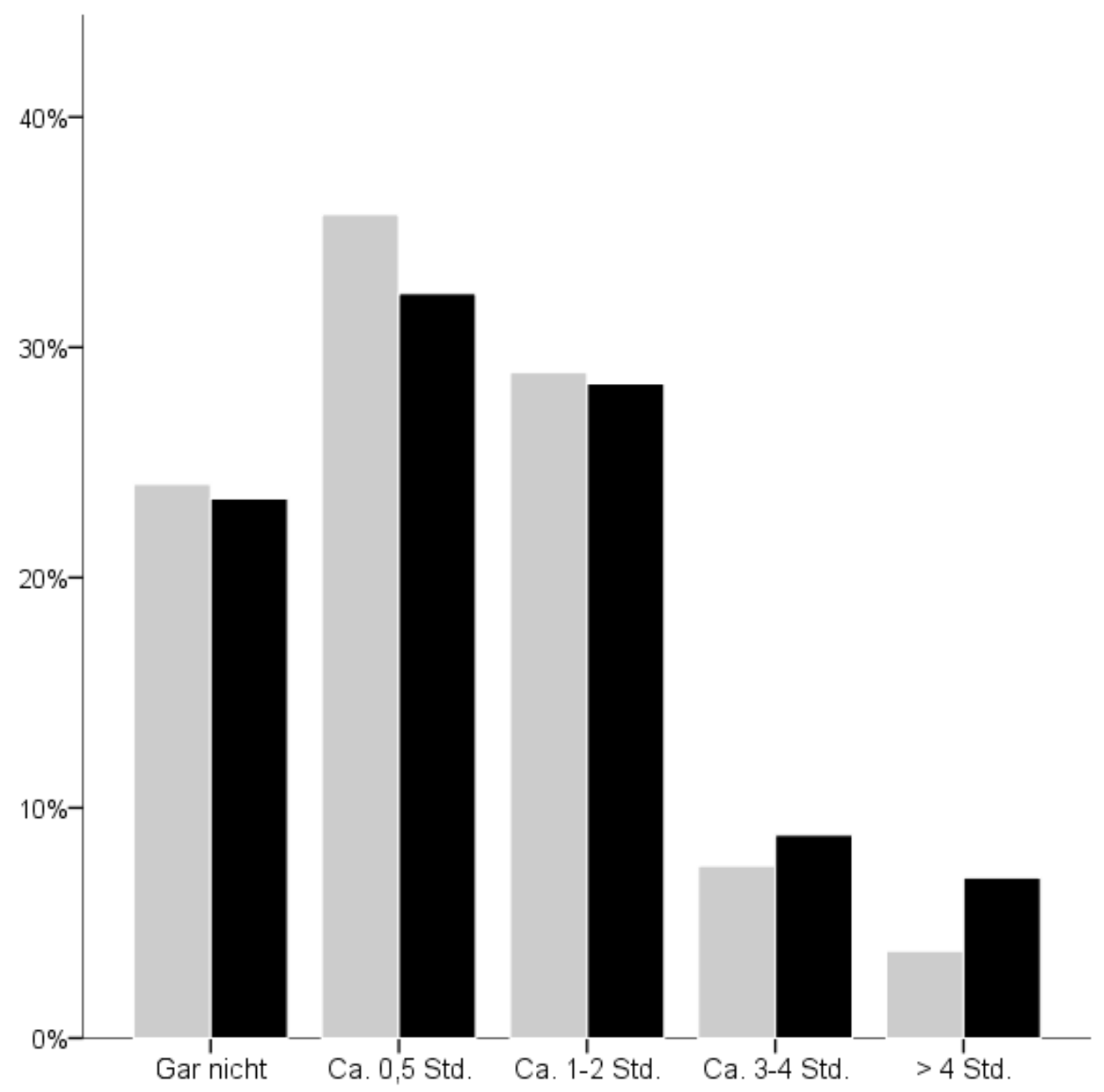

\section{Abb. 9 Häufigkeitsverteilung der Nutzung des Computers (in Stunden pro Tag)}

Die Darstellung bezieht sich auf den Computergebrauch in Prozent, jeweils getrennt für hypertensive (schwarz) und normotensive Jugendliche (grau).

Mit $p<0,01$ zeigt sich ebenfalls ein sehr signifikanter Unterschied bei dem Gebrauch des Handys: 79,6\% der Jugendlichen mit normalem arteriellen Blutdruck und $81,0 \%$ derjenigen mit erhöhtem arteriellen Blutdruck geben bis zu eine halbe Stunde, 11,0\% der Normotensiven und 12,8\% der Hypertensiven 1-2 
Stunden Nutzung pro Tag an. Über drei Stunden täglich gebrauchen 9,4\% der Normotensiven und 6,1\% der Hypertensiven ihr Handy. Zwar zeigt sich beim Rangniveau, dass die hypertensiven Jugendlichen einen etwas niedrigeren mittleren Rang aufweisen, also einen etwas niedrigeren Handykonsum haben, dennoch lässt sich zwischen den Gruppen kein eindeutig unterschiedlicher Trend bezüglich des Handygebrauchs feststellen. Beim Alkoholkonsum zeigt sich mit p<0,001 ein höchst signifikanter Unterschied: $70,0 \%$ der Jugendlichen mit erhöhtem arteriellen Blutdruck und 63,5\% derjenigen mit normalem Blutdruck haben schon einmal Alkohol getrunken. Hinsichtlich des Bierkonsums ist ebenfalls ein höchst signifikanter Unterschied mit $p<0,001$ festzustellen: Mehr als 5 Gläser pro Woche trinken $8,0 \%$ der Normotensiven und $11,8 \%$ der Hypertensiven, 1-4 Gläser pro Woche konsumieren $18,0 \%$ der Normotensiven und $23,4 \%$ der Hypertensiven. 1-3 Gläser pro Monat geben 15,1\% der Normotensiven im Vergleich zu 17,9\% der Hypertensiven an. Maximal ein Glas Bier pro Monat konsumieren 58,9\% der Normotensiven und 46,9\% der Hypertensiven. Insgesamt zeigen die Jugendlichen mit einem erhöhten arteriellen Blutdruck demnach einen höheren Bierkonsum. 


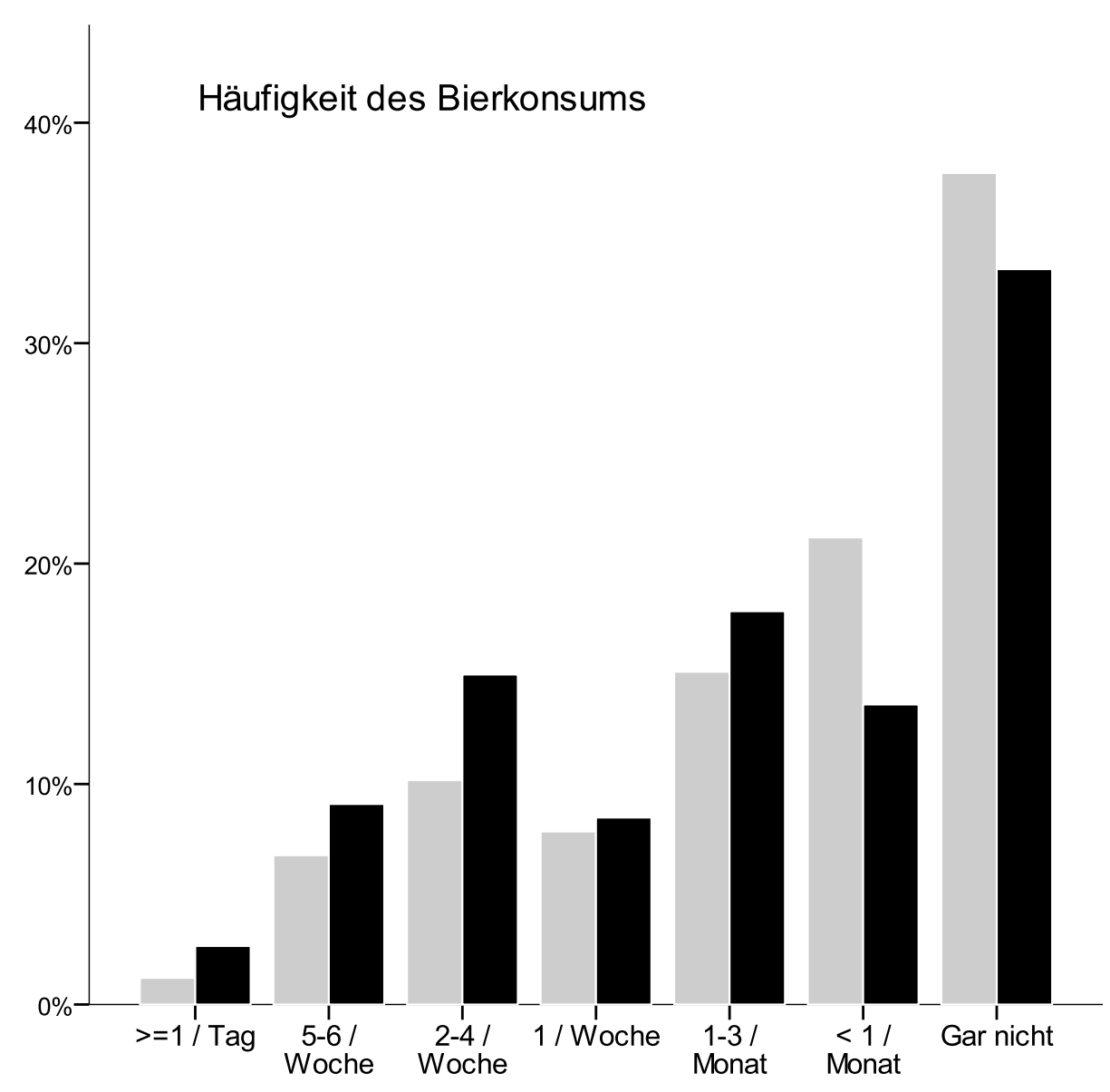

\section{Abb. 10 Vergleich des Alkoholkonsums zwischen hypertensiven und normotensiven Jugendlichen}

Die Darstellung zeigt den Bierkonsum im Vergleich von hypertensiven (schwarz) und normotensiven (grau) Jugendlichen, wobei die Prozentangabe sich insgesamt auf die jeweilige Gruppe bezieht. Die Angaben beinhalten die Anzahl an getrunkenen Gläsern.

Beim Wein- und Schnapskonsum ist kein signifikanter Unterschied zu finden (siehe Tabelle 15).

Auch beim Rauchen sowie dem Gebrauch von illegalen Drogen wie Marihuana, Haschisch, Ecstasy oder Aufputschmitteln weisen die beiden Gruppen keine signifikanten Unterschiede auf (Tabelle 15). 
Tab. 15 Verteilung des Alkohol- und Drogenkonsums sowie der körperlichen Aktivität bei hypertensiven und normotensiven Jugendlichen und Signifikanzangabe des Chi-Quadrat-Tests

\begin{tabular}{|c|c|c|c|c|}
\hline & $\begin{array}{l}\text { Gesamt- } \\
\text { kollektiv }\end{array}$ & $\begin{array}{l}\text { Hypertensive } \\
\text { Jugendliche }\end{array}$ & $\begin{array}{l}\text { Normotensive } \\
\text { Jugendliche }\end{array}$ & $\begin{array}{l}\text { Signifi- } \\
\text { kanz }\end{array}$ \\
\hline $\begin{array}{c}\text { Weinkonsum } \\
\text { (mind. } 5 \text { Gläser/Woche) }\end{array}$ & $\begin{array}{l}0,9 \% \\
N=40\end{array}$ & $\begin{array}{l}0,8 \% \\
N=4\end{array}$ & $\begin{array}{l}0,9 \% \\
N=36\end{array}$ & 0,145 \\
\hline $\begin{array}{l}\text { Schnapskonsum } \\
\text { (mind. } 5 \text { Gläser/Woche) }\end{array}$ & $\begin{array}{l}2,2 \% \\
N=98\end{array}$ & $\begin{array}{l}2,9 \% \\
N=15\end{array}$ & $\begin{array}{l}2,1 \% \\
N=84\end{array}$ & 0,062 \\
\hline Rauchen & $\begin{array}{l}20,4 \% \\
N=1553\end{array}$ & $\begin{array}{l}19,5 \% \\
N=158\end{array}$ & $\begin{array}{l}20,5 \% \\
N=1393\end{array}$ & 0,486 \\
\hline Illegale Drogen & & & & \\
\hline $\begin{array}{c}\text { Marihuana, Haschisch } \\
\text { (oft/mehrmals) }\end{array}$ & $\begin{array}{l}3,4 \% \\
N=256\end{array}$ & $\begin{array}{l}2,9 \% \\
N=23\end{array}$ & $\begin{array}{l}3,5 \% \\
N=233\end{array}$ & 0,911 \\
\hline $\begin{array}{c}\text { Ecstasy } \\
\text { (oft/mehrmals) }\end{array}$ & $\begin{array}{l}0,1 \% \\
N=10\end{array}$ & $\begin{array}{l}0 \% \\
N=0\end{array}$ & $\begin{array}{l}0,2 \% \\
N=10\end{array}$ & 0,689 \\
\hline $\begin{array}{l}\text { Aufputschmittel } \\
\text { (oft/mehrmals) }\end{array}$ & $\begin{array}{l}0,2 \% \\
N=15\end{array}$ & $\begin{array}{l}0,1 \% \\
N=1\end{array}$ & $\begin{array}{l}0,2 \% \\
N=14\end{array}$ & 0,446 \\
\hline $\begin{array}{c}\text { keine körperliche Aktivi- } \\
\text { tät }\end{array}$ & $\begin{array}{l}10,2 \% \\
N=767\end{array}$ & $\begin{array}{l}9,7 \% \\
N=78\end{array}$ & $\begin{array}{l}10,2 \% \\
N=689\end{array}$ & 0,192 \\
\hline
\end{tabular}

Hinsichtlich der körperlichen Aktivität ist nur ein Unterschied bei der Angabe zur eigenen körperlichen Leistungsfähigkeit zu finden. Hier ist das Ergebnis mit p<0,01 sehr signifikant: Als "sehr gut" oder "gut" bezeichnen 66,1\% der Jugendlichen mit einem normalen Blutdruck und $60,4 \%$ der Jugendlichen mit einem erhöhten Blutdruck ihre körperliche Leistungsfähigkeit. Eine „mittlere“ körperliche Leistungsfähigkeit geben 27,0 der Normotensiven und 30,6\% der Hypertensiven an. Als "nicht besonders gut" oder "gar nicht gut" schätzen 7,0\% 
der Normotensiven und 8,9\% der Hypertensiven ihre eigene körperliche Leistungsfähigkeit ein. Die Gruppe der Jugendlichen mit einem erhöhten arteriellen Blutdruck empfindet sich also insgesamt als weniger körperlich leistungsfähig.

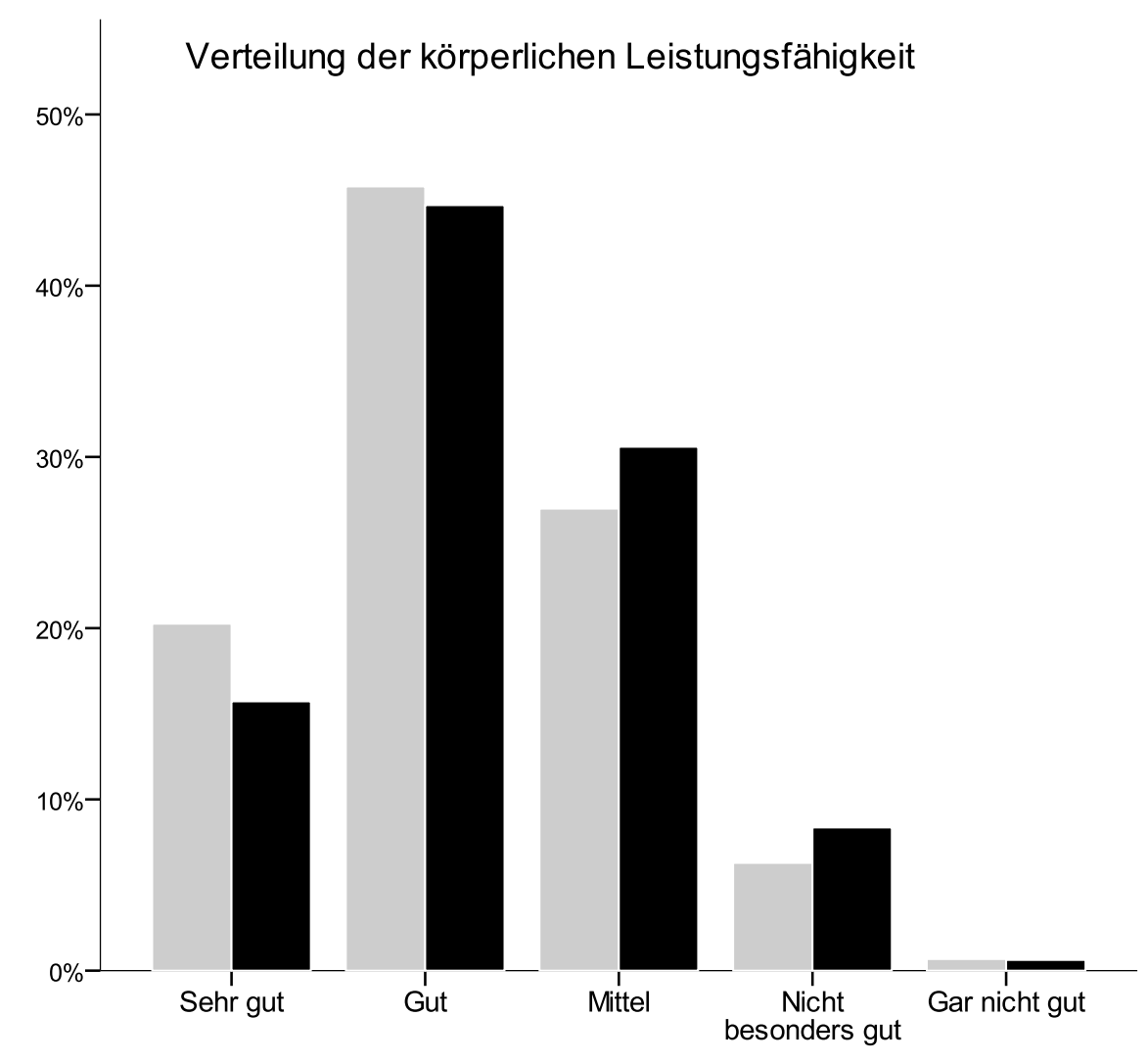

\section{Abb. 11 Selbsteinschätzung der körperlichen Leistungsfähigkeit}

Die Darstellung zeigt die Verteilung der körperlichen Leistungsfähigkeit von Hypertensiven (schwarz) und Normotensiven (grau). Die Prozentangaben beziehen sich insgesamt auf die jeweilige Gruppe.

Bei der körperlichen Aktivität zeigt sich jedoch kein Unterschied zwischen den Gruppen (siehe Tabelle 15, S.58).

Schließlich wurde noch das Allgemeinbefinden genauer betrachtet (siehe Tabelle 16, S.60): Keine Unterschiede sind hinsichtlich Schlafschwierigkeiten wie Einoder Durchschlafschwierigkeiten zu finden. Bei der Frage, ob die Jugendlichen eingeschränkt sind, Dinge zu tun, die die meisten Gleichaltrigen tun können, ergibt 
sich ein mit $p<0,05$ signifikanter Unterschied: 7,2\% der Hypertensiven und 4,9\% der Normotensiven geben hier Einschränkungen an. Bei Einschränkungen aufgrund gesundheitlicher Probleme zeigen sich dagegen keine signifikanten Unterschiede. Hinsichtlich des Auftretens von Störungen der körperlichen und emotionalen Entwicklung oder des Verhaltens sind keine signifikanten Gruppenunterschiede festzustellen. Zusätzlich wurde der Schuldurchlauf bei den Jugendlichen auf Differenzen überprüft. Mit $p<0,05$ zeigt sich hier ein signifikanter Unterschied: $85,6 \%$ der Jugendlichen mit einem erhöhten Blutdruck und 81,6\% derjenigen mit normalen Blutdruckwerten geben an, dass alle Klassen regulär durchlaufen wurden, wohingegen 14,1\% der Hypertensiven und 17,3\% der Normotensiven angeben, eine oder mehrere Klassen wiederholt zu haben. Die Jugendlichen mit erhöhten arteriellen Blutdruckwerten weisen demnach etwas weniger Schwierigkeiten beim Schuldurchlauf auf.

Tab. 16 Angaben zum Allgemeinbefinden im Vergleich zwischen hypertensiven und normotensiven Jugendlichen und Signifikanzangabe des ChiQuadrat-Tests

\begin{tabular}{|c|c|c|c|c|}
\hline & $\begin{array}{l}\text { Gesamt- } \\
\text { kollektiv }\end{array}$ & $\begin{array}{l}\text { Hypertensive } \\
\text { Jugendliche }\end{array}$ & $\begin{array}{l}\text { Normotensive } \\
\text { Jugendliche }\end{array}$ & $\begin{array}{c}\text { Sig- } \\
\text { nifikanz }\end{array}$ \\
\hline $\begin{array}{l}\text { Schlafschwierigkeiten } \\
\text { davon: }\end{array}$ & $\begin{array}{l}21,6 \% \\
N=1643\end{array}$ & $\begin{array}{l}20,4 \% \\
N=165\end{array}$ & $\begin{array}{l}21,7 \% \\
N=1472\end{array}$ & 0,417 \\
\hline Einschlafschwierigkeiten & $\begin{array}{l}75,7 \% \\
N=1205\end{array}$ & $\begin{array}{l}73,3 \% \\
N=118\end{array}$ & $\begin{array}{l}75,9 \% \\
N=1083\end{array}$ & 0,457 \\
\hline $\begin{array}{l}\text { Durchschlaf- } \\
\text { schwierigkeiten }\end{array}$ & $\begin{array}{l}37,7 \% \\
N=600\end{array}$ & $\begin{array}{l}36,3 \% \\
N=58\end{array}$ & $\begin{array}{l}37,8 \% \\
N=539\end{array}$ & 0,702 \\
\hline $\begin{array}{l}\text { Einschränkungen } \\
\text { davon: }\end{array}$ & $\begin{array}{l}5,2 \% \\
N=371\end{array}$ & $\begin{array}{l}7,2 \% \\
N=54\end{array}$ & $\begin{array}{l}4,9 \% \\
N=313\end{array}$ & 0,008 \\
\hline $\begin{array}{l}\text { Einschränkungen auf- } \\
\text { grund gesundheitlicher } \\
\text { Probleme }\end{array}$ & $\begin{array}{l}93,5 \% \\
N=334\end{array}$ & $\begin{array}{l}94,2 \% \\
N=49\end{array}$ & $\begin{array}{l}93,3 \% \\
N=281\end{array}$ & 0,813 \\
\hline $\begin{array}{l}\text { Emotionale, Entwick- } \\
\text { lungs- oder Verhaltens- } \\
\text { probleme }\end{array}$ & $\begin{array}{l}5,1 \% \\
N=360\end{array}$ & $\begin{array}{l}4,4 \% \\
N=33\end{array}$ & $\begin{array}{l}5,1 \% \\
N=324\end{array}$ & 0,401 \\
\hline
\end{tabular}




\subsubsection{Multivariate Überprüfung der Confounder im Modell 1}

Die gefundenen Confounder sollten weiter untersucht werden. Dabei wurden nur die Confounder im multivariaten Modell überprüft, die im Chi-Quadrat-Test für kategoriale Variablen eindeutig unterschiedliche Tendenzen zwischen den normotensiven und hypertensiven Jugendlichen erkennen ließen.

Bei den explorativ gewonnenen Confoundern handelt es sich um nominal- und ordinalskalierte Merkmale, die bei der binären logistischen Regressionsanalyse als kategoriale Variablen gekennzeichnet wurden. Es musste eine Merkmalsausprägung als Referenzkategorie bestimmt werden. Jede Kategorie der Einflussvariablen wurde anschließend mit der Referenzkategorie verglichen. Bei den Variablen „Einschränkungen“ (Fehldaten 572; 7,2\%) und „Bierkonsum“ (Fehldaten 3037; $39,5 \%)$ bestand eine hohe Anzahl an Fehldaten, da es sich bei beiden um Unterpunkte eines Fragenblocks handelt, die nur beantwortet wurden, wenn vorhergehende Fragen bejaht wurden. Um einen systematischen Fehler bei der multivariaten Testung in Modell 1 (sowie Modell 1b) zu vermeiden, wurde bei den beiden genannten Variablen eine fehlende Antwort definiert als „keine Einschränkung“ bzw. „kein Bierkonsum“.

Es erfolgte mithilfe der binären logistischen Regression die multivariate Überprüfung im Modell 1, bei dem die Confounder jeweils einzeln mit dem Alter, dem Geschlecht, dem BMI und dem sozialen Status überprüft werden. Die genauen Angaben der OR und der Signifikanzen sind den Tabellen I-XIII im Anhang $6.1 \mathrm{zu}$ entnehmen.

In der multivariaten Testung unterscheiden sich die Angaben zu bestehenden Einschränkungen zwischen hypertensiven und normotensiven Jugendlichen nicht mehr. Ebenso zeigt sich hinsichtlich des Gebrauchs des Computers kein signifikantes OR mehr. Im Gegensatz dazu ist bei der Nutzung des TV (1-2h/3-4h vs. gar nicht) mit $p \leq 0,01$ ein sehr signifikanter Unterschied festzustellen. Auch beim Bierkonsum zeigt sich in der multivariaten Testung (<1/Monat vs. gar nicht) mit $p<0,01$ ein sehr signifikantes OR. Bei der körperlichen Leistungsfähigkeit verfehlen die Ausprägungen „gar nicht gut“ vs. „sehr gut“ mit p=0,057 nur gering das Signifikanzniveau. Beim Schuldurchlauf erweist sich das $O R$ in der multivariaten Überprüfung mit $p \leq 0,001$ als höchst signifikant. 


\subsection{Multivariate Testung der relevanten Variablen im Modell 1b}

In der binären logistischen Regression sollten nun die Subskalen der Lebensqualität sowie die Subskalen der psychosozialen Probleme und Stärken jeweils einzeln im multivariaten Modell $1 \mathrm{~b}$ mit den explorativ gewonnenen Confoundern sowie den Variablen „Alter“, „Geschlecht“, „BMl“ und „sozialer Status“ als Prädiktoren des erhöhten Blutdrucks überprüft werden. Bei den explorativ gewonnenen Confoundern wurden nur die Variablen in das Modell $1 \mathrm{~b}$ einbezogen, die zwischen den beiden Gruppen einen eindeutigen Trend erkennen ließen. Beim Bierkonsum und bei bestehenden Einschränkungen wurde hier ebenfalls eine fehlende Antwort als „kein Bierkonsum“ oder „keine Einschränkungen“ definiert (s.o.).

\subsubsection{Lebensqualität und psychosoziale Probleme im multivariaten Modell $1 b$}

Die exakten OR sowie die zugehörigen Signifikanzangaben sind den Tabellen I bis XIII im Anhang zu entnehmen.

Bei den Elternangaben zeigen sich bezüglich der Subskalen der Lebensqualität mit $p<0,001$ höchst signifikante $O R>1$ in den Bereichen "Selbstwert" und „Familie“ sowie bei dem übergreifenden Messwert. Der Bereich „körperliches Wohlbefinden“ ist mit $p<0,05$ ebenfalls signifikant.

Die Elternangaben zu psychosozialen Schwierigkeiten sind in den Subskalen „Verhaltensauffälligkeiten“ und „Hyperaktivität" sowie bei dem Gesamtproblemwert mit $p<0,001$ höchst signifikant und zeigen $O R<1$. Darüber hinaus erweist sich das $O R<1$ des Bereichs „emotionale Probleme“ mit $\mathrm{p}<0,05$ als signifikant.

Bei den Eigenangaben zur Lebensqualität weist die Subskala „körperliches Wohlbefinden" sowie der übergreifende Messwert mit $p<0,001$ höchst signifikante OR $>1$ auf. Der Bereich „Familie“ ist mit $p<0,01$ sehr signifikant, die Subskala „Selbstwert" mit $p<0,05$ signifikant. Beide zeigen ein $O R>1$. Die Subskala „Hyperaktivität" weist bei den Eigenangaben zu den psychosozialen Problemen mit $p<0,001$ ein höchst signifikantes $O R<1$ auf. 
Sowohl bei den Elternangaben als auch bei den Eigenangaben geht ein höherer Score in den Subskalen der Lebensqualität mit einem größeren Risiko für einen erhöhten arteriellen Blutdruck einher. Bei den Subskalen zu den psychosozialen Schwierigkeiten zeigt ein höherer Score ein geringeres Risiko für erhöhte arterielle systolische und/oder diastolische Blutdruckwerte.

Die exakten OR der Kontrollvariablen (Alter, Geschlecht, BMI und sozialer Status) sowie der relevanten Confounder mit Signifikanzangaben sind den Tabellen I bis XIII im Anhang zu entnehmen.

\subsection{Güte der Modellanpassung und in die Analyse einbezogene Fälle}

In der multivariaten Analyse im Modell 1 variierte die Prozentzahl der einbezogenen Fälle (für die Berechnungen mit den Subskalen der Lebensqualität und der psychischen Gesundheit) zwischen 93\% und 96\%. Im Modell 1b wurden bei der Analyse zwischen 91 und 93\% der Fälle in die Berechnungen einbezogen.

Die Güte der Anpassung des Regressionsmodells wurde mit der LikelihoodFunktion bestimmt. Als Maß gilt der negative doppelte Wert des Logarithmus ($2 \mathrm{LL}$ ). Bei einer perfekten Modellanpassung würde der Funktionswert -2LL dem Wert 0 entsprechen.

Im Modell 1 zeigt sich ein minimaler -2LL-Wert von 4470,13 im Modell mit der Variablen „körperliches Wohlbefinden" aus den Eigenangaben und ein maximaler -2LL-Wert von 4629,68 im Modell mit der Variablen „emotionale Probleme“ aus den Elternangaben. Anhand der Klassifizierungstabellen wird deutlich, dass durch das Modell 1 minimal 68,6\% und maximal 70,5\% richtig negativ geschätzt werden. Richtig positiv werden dagegen minimal $57,1 \%$ und maximal 59,5\% der Fälle geschätzt.

Im Modell 1b zeigt sich ein minimaler -2LL-Wert von 4291,786 im Modell mit der Variablen „körperliches Wohlbefinden“ aus den Eigenangaben sowie einem maximalen -2LL-Wert von 4380,625 im Modell mit der Variablen „emotionale Probleme“ aus den Elternangaben. Die Klassifizierungstabellen zeigen, dass minimal 68,0\% und maximal $68,9 \%$ richtig negativ geschätzt werden. Minimal werden $62,0 \%$ und 
maximal 64,9\% richtig positiv geschätzt. Die Anpassungsgüte der verschiedenen Modelle kann den Tabellen I bis XIII im Anhang 6.1 entnommen werden.

\subsection{Lebensqualität und psychosoziale Probleme als abhängige Variablen im multivariaten Modell}

Während die bisherigen Analysen den Blutdruck als abhängige und die beschriebenen demographischen, psychosozialen und Verhaltensparameter als unabhängige Variablen betrachten, ist angesichts des querschnittlichen Studiendesigns eine klare Direktionalität der gefundenen Beziehungen nicht anzugeben. Der erhöhte Blutdruck kann zwar möglicherweise Folge bestimmter psychosozialer Faktoren sein; ebenso gut ist aber auch möglich, dass der erhöhte Blutdruck seinerseits das psychosoziale Befinden beeinflusst (vgl. Diskussion 4.2.4/ 4.2.5). Mit der linearen Regression sollte daher zum einen die Variable „Lebensqualität“, zum anderen die Variable „psychosoziale Probleme“ als abhängige Variable im multivariaten Modell auf einen Zusammenhang mit „arteriellem Blutdruck“ als Prädiktor getestet werden. Dabei sollte für Alter, Geschlecht, BMI und sozialen Status adjustiert werden. Alter und BMI wurden als metrische Variablen in die Berechnungen einbezogen. Der soziale Status wurde als ordinale Variable mit den Ausprägungen „niedriger“, „mittlerer" und „hoher Sozialstatus“ erfasst. Der arterielle Blutdruck wurde als dichotome Variable, d.h. systolisch und/oder diastolisch erhöhter Blutdruck erfasst. Die Güte der Anpassung wird durch das adjustierte R-Quadrat angegeben. Der Beta-Koeffizient zeigt den Einfluss der jeweiligen Variablen auf die abhängige Variable.

Zunächst wurde der übergreifende Messwert der Lebensqualität von Eltern- und Eigenangaben als abhängige Variable im multivariaten Modell auf eine Assoziation mit der Variablen „erhöhter arterieller Blutdruck“ als Prädiktor überprüft. Es fand eine Adjustierung für Alter, Geschlecht, BMI und sozialen Status statt: Auch in dieser Betrachtung erwies sich der arterielle Blutdruck als signifikanter Einflussfaktor auf die Lebensqualität sowohl für die Eigen- als auch für die Elternangaben. Ein erhöhter arterieller Blutdruck ist mit einer besseren Lebensqualität assoziiert (sie- 
he Tabelle 17). Die Beta-Koeffizienten und Signifikanzwerte der Variablen für die Adjustierung können ebenfalls Tabelle 17 entnommen werden.

Die Anpassungsgüte ist bei beiden Modellen gering, bei den Eigenangaben jedoch etwas besser als bei den Elternangaben.

Tab. 17 Übergreifender Messwert der Lebensqualität als abhängige Variable in der multivariaten Testung

\begin{tabular}{|l|l|l|l|l|}
\hline & \multicolumn{2}{|l|}{\begin{tabular}{l} 
Lebensqualität \\
\multicolumn{2}{|l|}{ (Eigenangaben) }
\end{tabular}} & \multicolumn{2}{l|}{$\begin{array}{l}\text { Lebensqualität } \\
\text { (Elternangaben) }\end{array}$} \\
\hline & $\begin{array}{l}\text { Beta- } \\
\text { Koeffizient }\end{array}$ & Signifikanz & $\begin{array}{l}\text { Beta- } \\
\text { Koeffizient }\end{array}$ & Signifikanz \\
\hline Erhöhter arterieller Blutdruck & 0,044 & $\mathrm{p}<0,001$ & 0,051 & $\mathrm{p}<0,001$ \\
\hline Alter & $-0,130$ & $\mathrm{p}<0,001$ & $-0,041$ & $\mathrm{p} \leq 0,001$ \\
\hline Geschlecht & $-0,124$ & $\mathrm{p}<0,001$ & $-0,028$ & $\mathrm{p}<0,02$ \\
\hline BMI & $-0,090$ & $\mathrm{p}<0,001$ & $-0,088$ & $\mathrm{p}<0,001$ \\
\hline Soziale Schicht & 0,042 & $\mathrm{p}<0,001$ & 0,046 & $\mathrm{p}<0,001$ \\
\hline $\begin{array}{l}\text { Adjustiertes } \\
\mathrm{R}^{2} / \text { Bestimmtheitsmaß }\end{array}$ & 0,050 & & 0,016 & \\
\hline
\end{tabular}

Zuletzt wurde der Gesamtproblemwert der psychosozialen Schwierigkeiten von Eltern- und Eigenangaben im multivariaten Modell auf einen Zusammenhang mit der Variablen „erhöhter arterieller Blutdruck“ als Prädiktor getestet. Wie bei der Lebensqualität sollte auch hier für Alter, Geschlecht, BMI und sozialen Status adjustiert werden: Auch in dieser Betrachtung erweist sich der erhöhte arterielle Blutdruck als signifikanter Prädiktor für psychosoziale Schwierigkeiten (siehe Tabelle 18). Ein erhöhter Blutdruck ist dabei mit weniger psychosozialen Problemen assoziiert. 
Die Beta-Koeffizienten und Signifikanzwerte von Alter, Geschlecht, BMI und sozialem Status aus dem Modell mit psychosozialen Problemen als abhängige Variable können der Tabelle 18 entnommen werden.

Die Anpassungsgüte ist bei den Eltern- und Eigenangaben gering, wobei sie bei den Elternangaben geringfügig besser ist.

Tab. 18 Gesamtproblemwert der psychosozialen Schwierigkeiten als abhängige Variable in der multivariaten Testung

\begin{tabular}{|l|l|l|l|l|}
\hline & \multicolumn{2}{|l|}{$\begin{array}{l}\text { Psychosoziale Probleme } \\
\text { (Eigenangaben) }\end{array}$} & \multicolumn{2}{l|}{$\begin{array}{l}\text { Psychosoziale Probleme } \\
\text { (Elternangaben) }\end{array}$} \\
\hline & $\begin{array}{l}\text { Beta- } \\
\text { Koeffizient }\end{array}$ & Signifikanz & $\begin{array}{l}\text { Beta- } \\
\text { Koeffizient }\end{array}$ & Signifikanz \\
\hline Erhöhter arterieller Blutdruck & $-0,040$ & $\mathrm{p} \leq 0,001$ & $-0,060$ & $\mathrm{p}<0,001$ \\
\hline Alter & $-0,009$ & $\mathrm{p}=0,446$ & $-0,129$ & $\mathrm{p}<0,001$ \\
\hline Geschlecht & 0,100 & $\mathrm{p}<0,001$ & $-0,116$ & $\mathrm{p}<0,001$ \\
\hline BMI & 0,119 & $\mathrm{p}<0,001$ & 0,103 & $\mathrm{p}<0,001$ \\
\hline Soziale Schicht & $-0,120$ & $\mathrm{p}<0,001$ & $-0,183$ & $\mathrm{p}<0,001$ \\
\hline $\begin{array}{l}\text { Adjustiertes } \\
\text { R2/Bestimmtheitsmaß }\end{array}$ & 0.042 & & 0,068 & \\
\hline
\end{tabular}




\section{Diskussion}

\subsection{Zentrale Erkenntnisse der vorliegenden Untersuchung}

Mit der vorliegenden Studie sollte die Assoziation eines erhöhten arteriellen Blutdrucks und der Lebensqualität in einer großen für Deutschland repräsentativen Stichprobe von 11- bis 17- jährigen Jugendlichen untersucht werden.

Analog zu vorhergehenden Studien konnte eine hohe Prävalenz erhöhter arterieller Blutdruckwerte im Jugendalter festgestellt werden. Dabei zeigte sich, dass vor allem Jungen häufiger hypertensiv waren, insbesondere mit zunehmendem Alter. Erwartungsgemäß verwiesen die Ergebnisse dieser Arbeit bei Jugendlichen mit erhöhten Blutdruckwerten auf ein ungünstigeres Freizeitverhalten mit einem stärkeren Medien- und Alkoholkonsum. Zusätzlich waren die Hypertensiven körperlich weniger leistungsfähig und häufiger adipös. Wider Erwarten zeigten sie einen besseren Schulerfolg mit selteneren Klassenwiederholungen.

Die zentrale Erkenntnis dieser Untersuchung war jedoch, dass Jugendliche mit erhöhten Blutdruckwerten von einer signifikant besseren Lebensqualität und psychischen Gesundheit in verschiedenen Bereichen zweier sehr gut validierter Messinstrumente berichteten. Darüber hinaus lagen bei Eigen- und Elternangaben übereinstimmende Ergebnisse vor. Auch die multivariate Testung sowie die umgekehrte Betrachtung mit der Lebensqualität als abhängiger Variablen bestätigten eine Assoziation eines erhöhten arteriellen Blutdrucks und eines besseren Wohlbefindens bei den Jugendlichen. 


\subsection{Bedeutendste Ergebnisse im Vergleich zu anderen Studien}

\subsubsection{Steigende Prävalenz des erhöhten arteriellen Blutdrucks im Ju- gendalter}

Anhand der Daten der großen deutschlandweiten Querschnittsstudie (KiGGS) wurde der Einfluss eines erhöhten arteriellen Blutdrucks auf die Lebensqualität von Jugendlichen untersucht. 10,7\% der 11- bis 17-Jährigen zeigten erhöhte Blutdruckwerte oberhalb des alters-, geschlechts- und größenadaptierten 95. Blutdruckperzentils. Dies wies auf eine hohe Anzahl an hypertensiven Jugendlichen mit einem größeren Anteil an männlichen Teilnehmern (61,3\%) hin. Beim sozialen Status waren keine Unterschiede in der Verteilung zwischen den Hyper- und Normotensiven nachzuweisen.

Hinsichtlich der arteriellen Hypertonie im Jugendalter gibt es sehr unterschiedliche Angaben zur Prävalenz, die von $3 \%$ bis $26 \%$ reichen (McNiece et al. 2007; Bancalari et al. 2011). Eine Querschnittsstudie von 1999 bis 2006 zeigte im Vergleich zu den Erkenntnissen der vorliegenden Untersuchung eine geringere Häufigkeit von 3,6\% Hypertonikern und 3,4\% Prähypertonikern. Hier handelte es sich jedoch zum einen um ein jüngeres Kollektiv (Durchschnittsalter 8,8 Jahre), zum anderen wurde entsprechend der korrekten Definition nur dann von einer arteriellen Hypertonie gesprochen, wenn zu drei unabhängigen Zeitpunkten erhöhte Blutdruckwerte vorlagen (Hansen et al. 2007). Diese wiederholte Bestimmung des Blutdrucks kann zu einer Reduktion des Anteils der hypertensiven Probanden führen: So wurde in einer Studie nachgewiesen, dass der initial bestimmte Anteil von 9,4\% Hypertensiven nach der Messung an drei verschiedenen Zeitpunkten auf $3,2 \%$ sank. Der Anteil der Prähypertoniker stieg dagegen von 9,5\% auf 15,7\% (McNiece et al. 2007).

Die Mehrheit der in den letzten Jahren publizierten Studien bestätigte jedoch den bei dem Kollektiv aus der KiGGS-Studie festgestellten Trend einer hohen Prävalenz eines erhöhten arteriellen Blutdrucks im Kindes- und Jugendalter. Dies zeigte sich nicht nur in Europa (12,8\%) (Maldonado et al. 2011), sondern auch in Lateinamerika (6,7-26\%) (Bancalari et al. 2011) und in Asien (9,4-24\%) (Durrani und Waseem 2011; Zhang und Wang 2011). Die Datenlage speziell für Deutschland ist stark eingeschränkt. Zwar gibt es einige wenige Untersuchungen zu diesem The- 
ma, diese sind jedoch untereinander und mit der vorliegenden Studie schlecht vergleichbar: eine geringere Kollektivgröße, eine abweichende Definition der arteriellen Hypertonie, die sich an Werten für Erwachsene orientiert, sowie die Verwendung neuer Grenzwerte, die sich von den im nationalen Vergleich üblichen unterscheiden, stellen Unterschiede zu der vorliegenden Dissertation dar. Die Angaben der Prävalenz reichen hier von 3\% bis 52\% (Klimm und Reuter-Kuhn 1994; Neuhauser et al. 2009; Flechtner-Mors et al. 2011). Diese große Spannweite lässt sich durch die oben genannten Punkte erklären und verweist auf die Notwendigkeit einer Untersuchung eines großen Kollektivs mit international vergleichbaren Definitionen und Standards, wie sie in der vorliegenden Arbeit stattgefunden hat. Bei dem Vergleich von zwei großen Querschnittsstudien in den USA, den National Health and Nutrition Examination Surveys (NHANES) von 1988-1994 und von 1999-2000, konnte bereits ein Anstieg des arteriellen Blutdrucks verzeichnet werden. Sowohl der durchschnittliche systolische $(1,4 \mathrm{mmHg})$ als auch der diastolische Blutdruckwert $(3,3 \mathrm{mmHg})$ stieg im Verlauf der beiden Studienzeitpunkte an. Auch nach Adjustierung des BMIs blieb die Zunahme weiterhin bestehen (Muntner et al. 2004), sodass die Ursache nicht allein in einer steigenden Adipositasprävalenz gesehen werden kann. Wie bei den Auswertungen der vorliegenden Dissertation konnte in der Analyse der NHANES ein höherer systolischer Mittelwert bei den männlichen im Vergleich zu den weiblichen Teilnehmern festgestellt werden. Nach den Ergebnissen der vorliegenden Studie nahm bei den Jungen mit zunehmendem Alter ebenfalls der Anteil der Hypertensiven zu, wohingegen bei den Mädchen schwankende Anteile bestanden. Dagegen zeigte sich in den NHANES bei den 8- bis 12-jährigen Mädchen ein größerer Anstieg des systolischen und diastolischen Blutdrucks mit zunehmendem Alter. Bei den 13- bis 17-Jährigen waren dann jedoch wie bei dem Kollektiv aus der KiGGS-Studie höhere Blutdruckwerte bei den Jungen festzustellen (Muntner et al. 2004).

In Anbetracht des steigenden Anteils an hypertensiven Jugendlichen sollten mögliche Ursachen für diesen Anstieg untersucht und somit überprüft werden, ob es möglicherweise bei Normo- und Hypertensiven Unterschiede in der psychischen Gesundheit und der Lebensqualität gibt. 


\subsubsection{Assoziation besserer Lebensqualität und psychischer Gesund- heit mit erhöhtem arteriellen Blutdruck}

Entgegen der Hypothese der vorliegenden Dissertation, dass hypertensive Jugendliche eine schlechtere Lebensqualität mit größeren psychosozialen Problemen aufweisen könnten, zeigte sich ein gegensätzlicher Trend: Bei den hypertonen Jugendlichen ergaben sich im Vergleich zu den normotonen sowohl bei der Lebensqualität als auch bei den psychosozialen Schwierigkeiten bessere Werte. Insgesamt ist der Zusammenhang von erhöhtem arteriellen Blutdruck und Wohlbefinden noch wenig erforscht und insbesondere die Datenlage für das Jugendalter ist rar. Allerdings konnte bei Erwachsenen in einigen Studien ein zu den Ergebnissen dieser Arbeit konträrer Zusammenhang festgestellt werden, bei dem die Hypertensiven ein geringeres Wohlbefinden angaben (Coelho et al. 1989; Dimenas et al. 1989; Battersby et al. 1995; Bardage und Isacson 2001; Erickson et al. 2001; Raskeliene et al. 2009; Saboya et al. 2010):

So zeigten auch Bardage und Isacson, dass Hypertoniker eine geringere Lebensqualität aufwiesen. Die Unterschiede waren besonders in physischen Gebieten wie der körperlichen Belastbarkeit und der körperlichen Beeinträchtigung des täglichen Lebens nachweisbar, weniger in psychischen Bereichen (Bardage und Isacson 2001; Erickson et al. 2001). Zusätzlich wurde ein gehäuftes Auftreten von körperlichen Beschwerden bei Hypertonikern nachgewiesen, sodass angenommen wurde, dass dadurch Einschränkungen bestanden, die die Lebensqualität negativ beeinflussten (Erickson et al. 2001).

Andere Untersuchungen konnten ergänzend eine Beeinträchtigung des psychischen Wohlbefindens feststellen (Coelho et al. 1989; Dimenas et al. 1989; Battersby et al. 1995). Li et al. (2005) wiesen beispielsweise in einer Studie aus China darauf hin, dass eine arterielle Hypertonie die allgemeine Gesundheitswahrnehmung beeinflusst, die sowohl die physische als auch die psychische Gesundheit widerspiegeln soll. Die Hypertoniker zeigten in allen Bereichen der erfassten gesundheitsbezogenen Lebensqualität (Selbstwertgefühl, Fitness, Copingstrategien, gesundheitliche Sorgen sowie Schmerz- und Stresswahrnehmung) schlechtere Werte: So wiesen sie im Gegensatz zu den hypertonen Jugendlichen in der KiGGS-Studie u. a. ein geringeres Selbstwertgefühl auf (Li et al. 2005). 
Anders als in der vorliegenden Arbeit, in der die hypertonen Jugendlichen weniger emotionale Probleme aufwiesen, konnte bei Erwachsenen eine Assoziation eines erhöhten arteriellen Blutdrucks mit Ängstlichkeit, emotionalem Leid, depressiver Verstimmung und einer geringeren Zufriedenheit festgestellt werden (Dimenas et al. 1989; Saboya et al. 2010). Es liegt nahe, dass es auch dadurch zu einer Beeinträchtigung von Lebensqualität und Wohlbefinden kommen kann.

Obwohl sich bei Erwachsenen wiederholt nachweisen ließ, dass ein erhöhter Blutdruck zu einer geringeren Zufriedenheit und einer schlechteren Lebensqualität führt, zeigte sich bei den hypertonen Jugendlichen in der KiGGS-Studie ein entgegengesetzter Trend.

Wie lassen sich diese Unterschiede erklären? Bereits 1987 äußerten Shapiro et al. die Vermutung, dass die Lebensqualität von Hypertonikern hauptsächlich durch drei Faktoren mitbestimmt wird: den Blutdruck selbst, die medikamentöse und nichtmedikamentöse Therapie sowie den Labeling-Effekt der Diagnose. Die Studien, die sich mit dem Zusammenhang von Blutdruck und Lebensqualität befassten, zeigten, dass die Erwachsenen meist über ihren Blutdruckstatus Bescheid wussten. Sie mussten damit zurechtkommen, an einer chronischen Erkrankung zu leiden. Im Gegensatz dazu handelte es sich bei den Jugendlichen aus der KiGGSStudie um einen Zufallsbefund. Die wenigsten von innen hatten eine Diagnose einer arteriellen Hypertonie mit den damit verbundenen Gedanken und Befürchtungen, wie sie oftmals bei Erwachsenen auftreten. Ein sogenannter "LabelingEffekt", der durch das Diagnostizieren einer Erkrankung hervorgerufen werden kann, führt zu einer Verschlechterung der Lebensqualität (Bloom und Monterossa 1981; Shapiro et al. 1987; Irvine et al. 1989; Mena-Martin et al. 2003; Li et al. 2005; Barger und Muldoon 2006; Hayes et al. 2008; Korhonen et al. 2011): Korhonen vermutete in einer Untersuchung von 2011, dass nicht der erhöhte Blutdruck per se zu einer Beeinträchtigung führt, sondern das Bewusstsein über die Erkrankung. So konnte er zeigen, dass die Hypertoniker, die über ihre Blutdruckwerte in Kenntnis waren, häufiger von einem reduzierten allgemeinen Gesundheitsstatus und einem verringerten körperlichen Wohlbefinden berichteten. Bei Hypertonikern, denen ihre Erkrankung nicht bewusst war, war dies dagegen nicht der Fall (Korhonen et al. 2011). Zusätzlich zu dem schlechteren körperlichen Befinden gaben die informierten Hypertoniker in anderen Studien ein geringeres psy- 
chisches Wohlbefinden an (Mena-Martin et al. 2003; Hayes et al. 2008). Außerdem konnte beobachtet werden, dass das Krankheitswissen auch mit depressiven Symptomen und Ängstlichkeit assoziiert war (Khang und Lynch 2011; Maldonado et al. 2011).

Barger und Muldoon (2006) konnten nachweisen, dass die Beeinflussung des Wohlbefindens abhängig vom Wissen über die Erkrankung ist, jedoch unabhängig von dem tatsächlichen Blutdruck oder der Medikamenteneinnahme: Selbst Probanden, die fälschlicherweise annahmen, an einer arteriellen Hypertonie erkrankt zu sein, gaben eine schlechtere Lebensqualität an. Das heißt, der sogenannte Labeling-Effekt ist unabhängig von dem tatsächlich bestehenden Blutdruck. Es scheint, als würden die Gedanken und Empfindungen, die mit dem Wissen verbunden sind, an einer chronischen Erkrankung zu leiden, viel mehr zu einer Beeinträchtigung des Wohlbefindens führen als der erhöhte Blutdruck als isolierter Faktor.

Diese starke Beeinflussung durch das Wissen über eine Erkrankung wurde wiederholt pathophysiologisch bestätigt: Bei den informierten Hypertonikern konnte im Stresstest ein stärkerer Anstieg des Blutdrucks, der Herzfrequenz sowie der Plasmakatecholamine nachgewiesen werden (Rostrup et al. 1990; Rostrup et al. 1991). Dies veranschaulicht, wie das alleinige Wissen um eine Erkrankung einen negativen Einfluss mit verstärkter Erregung und Anspannung haben kann. Die beschriebenen Studien weisen auf eine Beeinflussung der Lebensqualität und des Wohlbefindens hin, allein durch die Annahme erkrankt zu sein. Dieser negative Einfluss entfiel bei den von uns untersuchten Jugendlichen, da bei ihnen ein Zufallsbefund eines erhöhten arteriellen Blutdrucks vorlag.

Neben dem beschriebenen Labeling-Effekt wiesen die Erwachsenen noch weitere Unterschiede auf, die die Diskrepanz zwischen den Ergebnissen dieser Arbeit und vorherigen Studien erklären könnten: Bei Erwachsenen mit einer diagnostizierten Hypertonie wurde ein Großteil medikamentös behandelt und auch dies kann zu einer Beeinträchtigung des Wohlbefindens führen (Li et al. 2005; Trevisol et al. 2012): So zeigte sich, dass Hypertoniker unter medikamentöser Therapie eine schlechtere Lebensqualität angaben, unabhängig davon, ob ihr Blutdruck kontrolliert war oder nicht (Trevisol et al. 2012). Eine ungünstige Beeinflussung bestand vor allem hinsichtlich körperlicher und emotionaler Bereiche der Lebensqualität (Youssef et al. 2005). Zwar bewirken antihypertensive Medikamente eine reduzier- 
te Wahrnehmung von Symptomen, die durch die Erkrankung selbst verursacht sind wie beispielsweise Kopfschmerzen und Schwindel, dafür führen sie aber oftmals zu zusätzlichen Symptomen (Kjellgren et al. 1998). Diese sehr vielfältigen und von der Medikamentenklasse abhängigen Nebenwirkungen (Kjellgren et al. 1998) können zu einer Beeinträchtigung der Lebensqualität führen. Dies bestätigte sich in einer Studie von Bardage und Isacson (2000), bei der die Hypertoniker unter medikamentöser Therapie mit zusätzlichen Nebenwirkungen im Vergleich zu Probanden ohne Nebenwirkungen eine schlechtere Lebensqualität aufwiesen. Der größte negative Einfluss wurde durch Nebenwirkungen wie sexuelle Dysfunktion und emotionale Belastungen wie beispielsweise Schlaflosigkeit, Müdigkeit und Depression verursacht. Dieser Aspekt der negativen Beeinflussung durch Medikamentennebenwirkungen entfällt bei den Jugendlichen aus der KiGGS-Studie, die größtenteils nicht antihypertensiv behandelt wurden. Es bestand kein Unterschied zwischen den hypertensiven und normotensiven Jugendlichen bezüglich der Medikamenteneinnahme, sodass eine verstärkte Beeinträchtigung der Hypertoniker durch medikamentöse Nebenwirkungen unwahrscheinlich ist.

Weiterhin könnte die schlechtere Lebensqualität unter Medikamenteneinnahme bei den Erwachsenen dadurch bedingt sein, dass Patienten, die medikamentös therapiert werden, häufiger eine schwerere Form der arteriellen Hypertonie aufweisen (Hayes et al. 2008), denn es ist anzunehmen, dass diese oftmals mit einer schlechteren Lebensqualität assoziiert ist (Erickson et al. 2001). Die von uns betrachteten hypertensiven Jugendlichen wiesen jedoch eher leicht erhöhte Blutdruckwerte auf. Aufgrund ihres jungen Alters ist anzunehmen, dass sie noch kaum unter Folgeerkrankungen der Hypertonie litten und auch insgesamt selten zusätzliche schwere Erkrankungen aufwiesen, was ebenfalls zu einer reduzierten Lebensqualität hätte führen können (Bardage und Isacson 2000). So konnten gezeigt werden, dass ein geringes Wohlbefinden bei Hypertonikern stark durch Komorbiditäten beeinflusst ist und nicht durch den erhöhten arteriellen Blutdruck selbst: $20 \%$ der Varianz bei den Skalenwerten des Wohlbefindens konnte durch Komorbiditäten und nur 2\% durch den arteriellen Hypertonus erklärt werden (Lahad und Yodfat 1993).

Eine größere Zahl an Symptomen sowie eine stärkere Beeinträchtigung durch diese führt viel stärker zu einer Reduktion der Lebensqualität als der Blutdruckwert und die Erkrankungsdauer (Erickson et al. 2004). Bei den vorliegenden Auswer- 
tungen konnten bei den Jugendlichen keine Unterschiede hinsichtlich der Inanspruchnahme von medizinischer Versorgung und dem Auftreten anderer Erkrankungen nachgewiesen werden. Zwar gaben die hypertensiven Jugendlichen etwas häufiger an, unter Einschränkungen im Alltag zu leiden, bei detaillierteren Nachfragen zeigten sich jedoch keine signifikanten Unterschiede mehr. Auch die anderen Bereiche der deutschen Übersetzung des CSHCN unterschieden sich nicht signifikant, sodass hier die Definition eines speziellen Versorgungsbedarfs nicht erfüllt ist (Scheidt-Nave et al. 2007) und damit verbundene Einschränkungen der Jugendlichen ausgeschlossen werden können. Es ist anzunehmen, dass auch Belastungen durch Umstellungen im Alltag oder Begleiterkrankungen als negative Einflussfaktoren unwahrscheinlich sind. Offensichtlich sind nach einer kurzen Symptomdauer, wie es bei den Jugendlichen der Fall war, noch keine körperlichen Veränderungen als Folge des erhöhten arteriellen Blutdrucks vorhanden oder zumindest nicht wahrnehmbar.

Des Weiteren war in verschiedenen Studien eine Assoziation einer langen Krankheitsdauer mit einer geringen Lebensqualität festzustellen (Robbins et al. 1994; Youssef et al. 2005): je länger der bisherige Krankheitsverlauf, desto geringer das körperliche Wohlbefinden und desto stärker körperliche Beschwerden und Schlafschwierigkeiten (Robbins et al. 1994). Zusätzlich zeigte sich, dass bei Patienten mit zunehmendem Alter die Lebensqualität sank (Klocek und Kawecka-Jaszcz 2003). Beides könnte das schlechtere Wohlbefinden in den vorherigen Studien erklären, da die Erwachsenen im Unterschied zu den Jugendlichen zum einen bereits länger erkrankt und zum anderen älter waren.

Zusammenfassend ist festzustellen, dass Erwachsene sich normalerweise im Gegensatz zu Jugendlichen ihrer Diagnose einer arteriellen Hypertonie bewusst sind. Mögliche Befürchtungen von Langzeitfolgen wie Einschränkungen durch Folgeerkrankungen, Umstellungen des Alltags durch regelmäßige Arztbesuche und finanzielle Kosten sowie Nebenwirkungen durch antihypertensive Medikamente (Stein et al. 2002; Li et al. 2005; Khosravi et al. 2010) können zu einer Beeinträchtigung des Wohlbefindens führen. Außerdem leiden viele Patienten mit zunehmendem Alter und steigender Krankheitsdauer an Komorbiditäten. Neben diesen Aspekten kann aber auch allein das Wissen, erkrankt zu sein bzw. einen erhöhten Blutdruck aufzuweisen, zu einer Beeinträchtigung der Lebensqualität führen. 
Die vorliegenden mit den oben aufgeführten Studien zur Lebensqualität hypertoner Erwachsener nicht übereinstimmenden Ergebnisse sind damit durch mehrere Faktoren zu erklären: Zum einen waren sich die hypertensiven Jugendlichen aus der KiGGS-Studie der milden Erhöhung ihres Blutdrucks nicht bewusst, da sie bei Routineblutdruckmessungen zufällig entdeckt wurde. Demzufolge waren sie unbesorgt über mögliche Langzeitfolgen. Zum anderen nahmen sie meist keine Medikamente ein, die zu unerwünschten Nebenwirkungen führen können, und litten auch nicht an Folgeschäden oder Komorbiditäten.

\subsubsection{Zum Widerspruch zwischen ungünstigem Freizeitverhalten und dennoch besserer sozialer Angepasstheit}

Trotzdem können die oben genannten Faktoren nicht erklären, weshalb erhöhte arterielle Blutdruckwerte in dem betrachteten Kollektiv aus der KiGGS-Studie nicht nur nicht mit einer schlechteren, sondern sogar mit einer besseren Lebensqualität assoziiert waren.

Daher sollten die hypertensiven und normotensiven Jugendlichen auf weitere Unterschiede untersucht werden, wobei ihr Freizeit- bzw. Risikoverhalten sowie ihr allgemeines Befinden betrachtet wurden. Dabei zeigte sich, dass die Hypertensiven häufiger ein ungünstiges Freizeitverhalten und schlechtere Gesundheitsindikatoren aufwiesen.

So verbrachten sie zum einen mehr Zeit vor dem Computer und dem Fernseher und waren zum anderen häufiger adipös und weniger körperlich leistungsfähig als die normotensive Vergleichsgruppe. Dass Adipositas häufiger mit einem erhöhten arteriellen Blutdruck einhergeht, ist konsistent zu mehreren vorherigen Studien (Nielsen und Andersen 2003; Chen et al. 2006; Schwandt et al. 2010; Katona et al. 2011; Simonetti et al. 2011; Shi et al. 2012). Dabei zeigte sich, dass vor allem eine abdominale Fettleibigkeit einen Risikofaktor darstellt (Schwandt et al. 2010). Zusätzlich konnte nachgewiesen werden, dass Adipositas bei Mädchen früher als bei Jungen mit Blutdruckveränderungen einhergeht (Shi et al. 2012). Auch eine geringe körperliche Leistungsfähigkeit ist mit erhöhten arteriellen Blutdruckwerten assoziiert (Milligan et al. 1997; Li et al. 2005; Kollias et al. 2009; Shi et al. 2012). 
Es konnte sogar nachgewiesen werden, dass eine gute körperliche Fitness das Risiko, durch Adipositas an einer arteriellen Hypertonie zu erkranken, reduziert (Nielsen und Andersen 2003; Chen et al. 2006). Zusätzlich zu den schlechteren körperlichen Voraussetzungen wiesen die hypertensiven Jugendlichen ein ungünstigeres Freizeitverhalten auf und verbrachten mehr Zeit vor Computer oder Fernseher. Auch ein vermehrter Konsum von Medien kann mit einem erhöhten arteriellen Blutdruck einhergehen. Dabei zeigte sich, dass das Risiko zusätzlich von der Dauer des Gebrauchs abhängig war (Pardee et al. 2007; Goldfield et al. 2011). Außerdem gaben die hypertensiven Teilnehmer der KiGGS-Studie einen höheren Alkoholkonsum an, wohingegen sich wider Erwarten beim Rauchen sowie beim Konsum illegaler Drogen keine Unterschiede nachweisen ließen. Konsistent zu vorherigen Studien ist dabei der Zusammenhang von Alkoholkonsum und erhöhtem Blutdruck (Milligan et al. 1997; Jorgensen und Maisto 2008), obwohl einige Autoren diesen Zusammenhang auch negieren (Arslantas et al. 2008; Katona et al. 2011). Obgleich ein Zusammenhang von erhöhtem arteriellen Blutdruck und Rauchen naheliegt (Flouris et al. 2008; Kollias et al. 2009), konnte dies in dem untersuchten Kollektiv der KiGGS-Studie nicht bestätigt werden. Eine fehlende Assoziation mit dem Rauchen ist aber auch in anderen bereits vorliegenden Studien zu finden (Milligan et al. 1997; Arslantas et al. 2008; Katona et al. 2011). Möglicherweise sind die negativen Auswirkungen des Zigarettenkonsums nach so kurzer Zeit, wie es bei den Jugendlichen der Fall ist, noch nicht ausgeprägt. Problematisch an dem gesundheitsschädlichen Verhalten der hypertensiven Jugendlichen ist jedoch, dass es häufig bis in das Erwachsenenalter persistiert und somit das kardiovaskuläre Risiko massiv erhöht (Milligan et al. 1997). Interessanterweise stellt nicht nur das eigene Risikoverhalten einen Einflussfaktor dar, sondern auch das elterliche. So konnte ein Anstieg des Blutdrucks mit zunehmender Anzahl elterlicher Risikofaktoren wie beispielsweise Adipositas, arterieller Hypertonie und Rauchen verzeichnet werden. Ebenso erwies sich ein niedriger elterlicher Bildungsstatus als Prädiktor für erhöhte Blutdruckwerte beim Nachwuchs (Simonetti et al. 2011). Kein Einfluss durch den sozialen Status bestand dabei in anderen Studien (Katona et al. 2011; Shi et al. 2012). So konnte auch in dem KiGGSKollektiv bei den hypertensiven Jugendlichen kein größerer Anteil mit niedrigem Sozialstatus gefunden werden. Die Verteilung erwies sich als ausgeglichen. 
Obwohl sich das Schlafverhalten in vorhergehenden Studien als blutdruckbeeinflussend herausgestellt hat mit einem gesteigerten Risiko für erhöhte arterielle Blutdruckwerte bei einer schlechten Schlafqualität (Javaheri et al. 2008), konnten hier bei der Betrachtung des Allgemeinbefindens zwischen den Normo- und Hypertensiven keine Unterschiede in Bezug auf Schlafschwierigkeiten festgestellt werden. Diese fehlende Assoziation mit dem arteriellen Blutdruck stimmt mit den Ergebnissen einer anderen Studie überein, die bei 3- bis 10 -jährigen Kindern ebenfalls keine Beeinflussung feststellte (Bayer et al. 2009). Offensichtlich ist bei Kindern und Jugendlichen die Auswirkung einer schlechten Schlafqualität noch nicht ausgeprägt genug, sodass noch keine Unterschiede zwischen Hyper- und Normotensiven erkennbar sind.

Obwohl Adams et al. (2010) in einer Untersuchung darlegten, dass hypertensive Kinder und Jugendliche häufiger sowohl Lernschwierigkeiten als auch ADHS aufwiesen, konnte in dem sehr viel größeren Kollektiv der 11- bis 17-Jährigen aus der KiGGS-Studie das Gegenteil nachgewiesen werden: Hier zeigten die Hypertensiven interessanterweise einen besseren Schulerfolg. Seltener als ihre normotensiven Mitschüler mussten sie eine Klasse wiederholen. Sie wiesen damit häufiger einen regulären Schuldurchlauf auf. Außerdem beklagten sich sowohl die hypertensiven Jugendlichen selbst als auch ihre Eltern seltener über Schwierigkeiten mit hyperaktiven Verhaltensproblemen. Entsprechend wiesen die Hypertensiven seltener als die Normotensiven ein vom Arzt oder Psychologen diagnostiziertes ADHS auf. Die geringeren Lernschwierigkeiten bei den hypertensiven Teilnehmern aus der KiGGS-Studie könnten dadurch erklärt werden, dass weniger zusätzliche Hyperaktivitätsprobleme bei ihnen vorlagen, denn Lernschwierigkeiten und Aufmerksamkeits-Hyperaktivitätsprobleme stellen häufig ein Kontinuum dar (Mayes et al. 2000). Im Gegensatz zu der vorliegenden Arbeit wurde in der Studie von Adams et al. (2010), in der bei Hypertonikern häufiger ADHS und Lernschwierigkeiten auftraten, nur dann von „ADHS“ gesprochen, wenn die Kinder und Jugendlichen eine entsprechende Medikation erhielten. Diese Betrachtung erscheint recht einseitig, zumal die Medikation selbst zu einer Blutdruckerhöhung führen kann, sodass die Assoziation zwischen Blutdruck und ADHS hier sekundär durch Medikamentennebenwirkungen zustande gekommen sein könnte.

Der bessere Schulerfolg sowie die geringeren Hyperaktivitätsprobleme der hypertensiven Jugendlichen könnten auf der einen Seite darauf hinweisen, dass ein er- 
höhter Blutdruck mit einer besseren psychosozialen Anpassung einhergeht. Auf der anderen Seite zeigten die Hypertensiven ein ungünstigeres Freizeitverhalten mit einem größeren Medien- und Alkoholkonsum, einer geringeren körperlichen Fitness und einem häufigeren Auftreten von Adipositas. Diese Diskrepanz zwischen dem ungünstigen Gesundheitsverhalten auf der einen Seite und dem besseren Schulerfolg sowie der besseren Lebensqualität mit weniger psychosozialen Schwierigkeiten auf der anderen Seite erfordert eine Erklärung:

Obwohl es noch spekulativ erscheint, könnte angenommen werden, dass sozialer Anpassungsdruck an die Ansprüche des Umfelds Leistungsangst und autonome Erregung in den Jugendlichen begünstigt. Obwohl die Erfahrung von Schulerfolg und das Erlangen von Bildung das Selbstwertgefühl und die psychosoziale Funktionsfähigkeit der Jugendlichen in der Regel steigern, wird dies oftmals nur auf Kosten von stärker wahrgenommenem Stress erreicht. In diesem Zusammenhang könnte der erhöhte arterielle Blutdruck als eine Konsequenz von objektiven Stressoren wie Leistungs- und Anpassungsdruck interpretiert werden. In der Tat beschreiben Nyklicek et al. (1996) in ihrem Review, dass diese häufig mit einem erhöhten Blutdruck assoziiert sind. Ein hohes Maß an objektiven Stressoren bei den hypertensiven Jugendlichen könnte auch ihr ungünstiges Gesundheitsverhalten erklären: Längere Fernseh- und Computerzeiten, Überernährung und Alkoholkonsum wären somit als Copingstrategie für Belastungen durch (teilweise eventuell bereits verinnerlichte) elterliche Anforderungen und Druck durch das Umfeld zu interpretieren.

\subsubsection{Hypothese der „learned hypertension“}

Es erscheint plausibel und ist auch vielfach belegt, dass Episoden von gesteigertem Stress zu einem kurzfristigen Blutdruckanstieg führen können (Ewart und Kolodner 1994; Beatty und Matthews 2009; Ewart et al. 2011). Mehrfach konnte nachgewiesen werden, dass Belastungen bei Jungen eine stärkere Blutdruckreaktion als bei Mädchen bedingen (Anderson und McNeilly 1991). Bei den Jugendlichen aus der KiGGS-Studie konnten ebenfalls häufiger bei männlichen Teilnehmern erhöhte Blutdruckwerte festgestellt werden. Stabile individuelle Eigenschaf- 
ten einer Person beeinflussen die Wahrnehmung des sozialen Umfelds und bestimmen dadurch das eigene Handeln. Bestimmte Charaktereigenschaften prädisponieren zu einer intensiveren oder schnelleren Blutdruckreaktion auf Stress und können als „persönliche Diathese“ mit einem gesteigerten kardiovaskulären Risiko betrachtet werden (Ewart und Kolodner 1994; Ewart et al. 1998). So stellt beispielsweise die Eigenschaft, unbedingt von anderen akzeptiert werden zu wollen, eine große Vulnerabilität gegenüber Blutdruckreaktionen dar (Ewart et al. 1998). In Anbetracht der Ergebnisse dieser Arbeit, wonach die hypertensiven Jugendlichen einen besseren Schulerfolg und geringere Probleme mit Hyperaktivität und anderen psychosozialen Problemen aufwiesen, kann von einer besseren sozialen Angepasstheit und einem damit möglicherweise verbundenen Bedürfnis nach größerer sozialer Akzeptanz dieser Jugendlichen gesprochen werden. Der Druck, anderen gefallen zu wollen und sich an Anforderungen der Gesellschaft anpassen zu müssen, könnte bei innen zunächst zu vorübergehenden Blutdruckanstiegen geführt haben. In weiteren Studien zeigte sich, dass psychosozialer Stress nicht nur einen kurzfristigen Blutdruckanstieg bedingen kann, sondern auch einen Beitrag zur Entwicklung einer arteriellen Hypertonie leistet (Gasperin et al. 2009; Spruill 2010; Carroll et al. 2012). Einen begünstigenden Faktor dabei könnten die stressreduzierenden Eigenschaften eines erhöhten Blutdrucks über die vagalen Afferenzen von Barorezeptoren darstellen (Dworkin et al. 1979; Dworkin et al. 1994; Elbert et al. 1994; Rau und Elbert 2001).

Physiologisch sind die Barorezeptoren in Carotissinus und Aortenbogen an der Regulation des Blutdrucks beteiligt, indem sie kardioinhibitorisch und sympathoinhibitorisch wirken, um dadurch zu schnelle und zu starke Blutdruckschwankungen abzumildern. Zusätzlich zu diesen kardiovaskulären Eigenschaften führen sie jedoch auch zu inhibitorischen Effekten im zentralen Nervensystem (Dworkin et al. 1994): So wurden eine Synchronisation im EEG (Bonvallet et al. 1953), eine geringere kortikale Erregbarkeit, eine abgeschwächte Schreckreaktion sowie ein erleichtertes Einschlafen (Rau und Elbert 2001) nachgewiesen. Zusätzlich konnte eine Angst- (Adam et al. 1963) und Schmerzreduktion mit einer Barorezeptoraktivierung (Maixner 1991; Dworkin et al. 1994; Rau und Elbert 2001) in Verbindung gebracht werden. Die zunächst im Tierversuch an Ratten nachgewiesene Unterdrückung nozizeptiver Reflexe durch Barorezeptorstimulation (Dworkin et al. 1979; Randich und Maixner 1986) konnte ebenfalls bei Menschen belegt 
werden (Apkarian et al. 1989; Dworkin et al. 1994; Rau und Elbert 2001). Auch bei innen zeigte sich eine reduzierte Wahrnehmung sowohl akuter als auch chronischer Schmerzen. Ein Zusammenhang mit dem endogenen Opiatsystem konnte im Tierversuch gefunden werden, da die hypertensive Hypoalgesie durch die Gabe von Opiatantagonisten aufgehoben werden konnte (Bonvallet et al. 1953; France 1999).

Diese zusätzlichen Auswirkungen einer Barorezeptorstimulation könnten einen positiven Einfluss auf die Lebensqualität haben. So wurde nachgewiesen, dass die Vagusstimulation einen stimmungsaufhellenden Effekt bei therapierefraktärer Depression haben kann (siehe 4.3, S. 85). Die Stimulation der Barorezeptoren stellt möglicherweise einen Verstärkermechanismus dar, der den kurzfristigen Blutdruckanstieg in stressauslösenden Situationen durch ein verbessertes Wohlbefinden belohnt. Entsprechend den Gesetzen des operanten Konditionierens wird das Verhalten, das zum Blutdruckanstieg geführt hat, verstärkt. Mit der Zeit kann so der kurzfristige Blutdruckanstieg in einen ständig erhöhten Blutdruck übergehen. Personen mit einem stärkeren Barorezeptoreinfluss auf zerebrale Strukturen haben ein größeres Risiko für die Entwicklung einer „learned Hypertension“ (Rau und Elbert 2001). In einer longitudinalen Studie testeten Elbert et al. (1994) die Hypothese, dass bei Personen mit sensiblen Barorezeptoren aus einem vorübergehenden Blutdruckanstieg als erlernte Antwort auf stressauslösende Umgebungsfaktoren ein langfristiger Blutdruckanstieg entstehen kann. Dabei stellten sie fest, dass die Intensität der Schmerzreduktion durch die Barorezeptoraktivität sowie der tägliche selbst empfundene Stresspegel mit einem späteren Blutdruckanstieg assoziiert sind. Das bedeutet, dass sensible Barorezeptoren eine persönliche Diathese darstellen, die dafür prädisponieren, eine Blutdruckerhöhung als Copingstrategie zu erlernen. Begünstigend wirken dabei ein hoher sozialer Druck und ein ausgeprägtes Stressempfinden, da in diesen Situationen die positiven Eigenschaften einer Barorezeptoraktivierung in Form von Stress- und Angstreduktion besonders zum Tragen kommen (Elbert et al. 1994). Damit kann auch die Blutdruckerhöhung bei den hypertensiven Jugendlichen mit guter sozialer Angepasstheit aus der KiGGS-Studie hypothetisch erklärt werden: Chronische Anforderungen der Umgebung lösten bei Jugendlichen mit sensiblen Barorezeptoren zunächst einen graduellen Blutdruckanstieg aus. Neben dem ungünstigen Freizeitverhalten diente der erhöhte Blutdruck als Copingstrategie gegen Stress. Die Jugendlichen fühlten 
sich besser und waren weniger angespannt. Die Blutdruckerhöhung bewirkte eine Belohnung, was zu ihrer Chronifizierung geführt haben könnte und gegebenenfalls zur Entwicklung einer arteriellen Hypertonie beitragen kann.

Eine Erklärung für die Unterschiede zwischen den Ergebnissen der vorliegenden Dissertation und den Resultaten der Mehrzahl der vorherigen Studien an Erwachsenen könnte demnach ein stärker wahrgenommener stressreduzierender und zufriedenstellender Effekt der Barorezeptorstimulation bei Jugendlichen sein. Im Gegensatz zu Erwachsenen sind diese durch Begleitfaktoren weniger beeinträchtigt: Folgeerkrankungen, Medikamentennebenwirkungen und Sorgen aufgrund des Krankheitswissens belasteten die Erwachsenen aus den früheren Studien eventuell so sehr, dass der positive Einfluss der Barorezeptorstimulation möglicherweise überlagert wurde. Einige wenige Studien weisen aber auch bei Erwachsenen auf eine positive Beeinflussung durch Barorezeptorstimulation hin: Bereits 1988 fanden Winkleby et al. eine inverse Assoziation des arteriellen Blutdrucks mit selbst wahrgenommenen Stressoren (im Gegensatz zu äußeren objektiv messbaren): Im Rahmen einer Querschnittsstudie wurden dabei 1428 Busfahrer in San Francisco zu selbst wahrgenommenen psychosozialen Stressoren befragt. Es konnte nachgewiesen werden, dass von hypertensiven Probanden, selbst nach Adjustierung möglicher Confounder, weniger subjektiv empfundene berufliche Probleme benannt wurden. Unter gewissen Bedingungen scheint demnach auch bei Erwachsenen eine inverse Assoziation des arteriellen Blutdrucks mit subjektiven Problemen bzw. Stressoren möglich (Winkleby et al. 1988). Zusätzlich zeigte sich in einer longitudinalen Studie, dass Probanden, die im Verlauf hochnormale oder erhöhte Blutdruckwerte entwickelten, insgesamt eine größere Zufriedenheit mit weniger Stress und geringeren subjektiven Belastungen aufwiesen (Jenkins et al. 1985; Nyklicek et al. 1996). Dies stimmt mit der Beobachtung überein, dass Personen mit einem erhöhten Risiko für eine arterielle Hypertonie oder mit einem frühen Stadium einer asymptomatischen arteriellen Hypertonie dazu neigen, eine „nicht-klagende“ Lebenseinstellung mit Herunterspielen von Stressoren zu zeigen (Theorell 1990; Nyklicek et al. 1996). 


\subsubsection{Affektrepression als mögliche Teilursache der Blutdruck- erhöhung}

In Anbetracht des Querschnittsdesigns der vorliegenden Studie lässt sich nicht sagen, ob nicht auch eine weitere Erklärung der Pathogenese des erhöhten Blutdrucks in Betracht kommt, bei der der erhöhte Blutdruck nicht Ursache der subjektiv wahrgenommenen besseren Lebensqualität ist, sondern Folge der zu dieser Wahrnehmung führenden psychischen Prozesse: Durch die unbewusste Verdrängung belastender Gefühle soll das Bewusstsein vor negativen Einflüssen geschützt werden (Mann 2012). Diese Affektrepression führt zu weniger selbst wahrgenommenen psychosozialen Problemen und einer verbesserten Lebensqualität, da belastende Emotionen nicht bewusst erlebt werden. In verschiedenen Studien ließ sich nachweisen, dass die beschriebene Verdrängungsneigung mit dem Auftreten einer arteriellen Hypertonie assoziiert ist (Linden und Feuerstein 1983; Sommers-Flanagan und Greenberg 1989): Verdrängte negative Affekte könnten so eine Teilursache für die Entwicklung erhöhter arterieller Blutdruckwerte darstellen, indem sie möglicherweise über das autonome Nervensystem Einfluss nehmen (Mann 2012).

Dies könnte zu der Vermutung führen, dass die in der KiGGS-Studie befragten Jugendlichen, die großen Belastungen ausgesetzt waren, die beschriebenen unbewussten Verdrängungsmechanismen einsetzten. Bei Befragungen könnten sie somit bessere Werte angegeben haben, da sie sich subjektiv besser fühlten. Aufgrund der Akkumulation der verdrängten negativen Affekte könnte sich dann im Verlauf aber ein erhöhter Blutdruck entwickelt haben.

Infolge der Verdrängung belastender Emotionen als Copingstrategie wirken die Probanden ausgeglichener und geben selbst bei erheblichem Stress kaum Belastungen an (Mann 2012). Interessanterweise erklärten aber auch die Eltern der hypertensiven Jugendlichen in der KiGGS-Studie, dass ihre Kinder weniger gestresst, weniger hyperaktiv und zufriedener seien als die normotensiven Jugendlichen. Dies weist darauf hin, dass nicht nur die Jugendlichen selbst ihre negativen Affekte unterdrückten, sondern dass auch ihre Eltern sie als besser angepasst wahrnahmen. Damit liegt eine mögliche Interaktion zwischen Eltern und Kindern bei der Entstehung eines erhöhten arteriellen Blutdrucks über die Verdrängung von negativen Emotionen nahe. 


\subsection{Bedeutung für den klinischen Alltag}

Die hohe Prävalenz erhöhter arterieller Blutdruckwerte in dem jugendlichen Kollektiv der KiGGS-Studie weist gemeinsam mit Literaturbefunden auf eine zunehmende Bedeutung der arteriellen Hypertonie bereits im Jugendalter hin. Diese Thematik sollte stärker ins Bewusstsein rücken und die routinemäßige Blutdruckmessung wie beim Erwachsenen als Vorsorgemaßnahme in den pädiatrischen Alltag integriert werden. Dabei sollte die korrekte Anwendung der geschlechts-, alters- und größenadaptierten Grenzwerte beachtet werden, was die korrekte Einteilung in hypertensiv und normotensiv im Vergleich zum Erwachsenen etwas erschwert, zumal sich aus KiGGS andere Normwerte ergeben würden (Neuhauser et al. 2011) als aus der der vorliegenden Arbeit zugrunde liegenden amerikanischen Referenzstichprobe (NHBPEP 2004). In jedem Fall ergibt sich aus dem laut Literatur steigenden Anteil hypertensiver Jugendlicher, dass Vorsorgemaßnahmen bereits im Kinder- und Jungendalter sinnvoll sind.

Die festgestellte Assoziation eines erhöhten Blutdrucks mit einer besseren Lebensqualität bei Jugendlichen könnte mit der Hypothese der erlernten arteriellen Hypertonie erklärt werden: Ein stressbelasteter Alltag mit einem starken sozialen Druck, erfolgreich und sozial angepasst zu sein, könnte bei prädisponierten Personen zur Erlernung einer arteriellen Blutdruckerhöhung als Copingstrategie führen. Aus dem Entstehungsmechanismus der „learned Hypertension“ ergibt sich die Notwendigkeit frühestmöglicher Interventionen, um einem steigenden Anteil an hypertensiven Personen aufgrund von Stressfaktoren im Alltag entgegenzuwirken. Das rechtzeitige Erlernen alternativer stressreduzierender Maßnahmen könnte den Kreislauf von kurzfristiger Blutdruckerhöhung mit verbessertem Wohlbefinden und daraus resultierender langfristiger arterieller Hypertonie an seinem Entstehungspunkt unterbrechen. Zusätzlich könnte damit auch verhindert werden, dass die Jugendlichen ein ungünstiges Freizeitverhalten mit hohem Medienkonsum und regelmäßigem Alkoholgenuss als Ausgleich für ihren anstrengenden Alltag entwickeln und damit zusätzlich die Entstehung einer Hypertonie fördern.

Auch das Erklärungsmodell der Affektrepression, das die unbewusste Verdrängung negativer Emotionen als Teilursache der Entstehung erhöhter Blutdruckwerte vermutet, spricht für die Bedeutung des Vermittelns psychosozialer Fertigkeiten 
bei der Behandlung und Prävention der arteriellen Hypertonie: Indem Patienten erlernen, wie sie ihre Gefühle besser wahrnehmen und zum Ausdruck bringen, könnte die Akkumulation negativer Affekte und ein dadurch eventuell bedingter Blutdruckanstieg verhindert werden. Neben dem Ansatzpunkt der psychotherapeutischen Intervention beschreibt Mann (2012) eine weitere klinische Bedeutung des Erklärungsmodells der Affektrepression: Da Emotionen das sympathische Nervensystem beeinflussen können, ließe sich vermuten, dass psychische Faktoren auch an der Entstehung einer neurogenen Hypertonie mitbeteiligt sind. Die Klassifizierung der dafür relevanten psychischen Faktoren könnte bei der Identifizierung der entsprechenden Patienten hilfreich sein. Bei diesen sollten medikamentöse Therapien am sympathischen Nervensystem angreifen, um kausal zu wirken (Mann 2012).Sollte sich der Zusammenhang von Wohlbefinden und arteriellem Blutdruck in weiteren Studien bestätigen, dann könnte eine Erklärung für die oftmals schlechte Adhärenz der Patienten bei der Einnahme antihypertensiver Medikamente die Unterbrechung der Interaktion von Barorezeptoren und ZNS sein. Das gesteigerte Wohlbefinden, vermittelt über vagale Afferenzen der Barorezeptoren bei einem Blutdruckanstieg, wird durch die Medikamente verhindert und die Patienten verspüren eine geminderte Lebensqualität (Bardage und Isacson 2000; Bremner 2002; Trevisol et al. 2012). Dies könnte eine schlechte Adhärenz bei der Einhaltung der antihypertensiven Therapie begünstigen (Trevisol et al. 2012).

Als alternative therapeutische Interventionsmöglichkeit ist die elektrische Barorezeptorstimulation zur Reduktion des Blutdrucks in Erprobung. Dabei wird über eine chronische elektrische Aktivierung der Carotissinuswand der afferente Teil des Barorezeptorreflexes stimuliert, was zu einer Blutdrucksenkung führen soll (Makris et al. 2010; $\mathrm{Ng}$ et al. 2011).

Im Tierversuch hat sich bereits bestätigt, dass eine anhaltende Barorezeptorstimulation zu einer kontinuierlichen Blutdrucksenkung führt. Zusätzlich hatte diese Stimulation auch eine Reduktion der gesteigerten Sympathikusaktivität im Rahmen einer adipositasassoziierten arteriellen Hypertonie zur Folge (Lohmeier et al. 2004; Lohmeier et al. 2007).

Es konnte nachgewiesen werden, dass die chronische Stimulation auch beim Menschen kardiovaskuläre Auswirkungen hat: Veränderungen der Herzfrequenzvariabilität und der Herzfrequenzturbulenzen korrelierten mit einem Abfall des arte- 
riellen Blutdrucks und wiesen auf eine Abnahme der Sympathikus- bei einer Zunahme der Parasympathikusaktivität hin (Wustmann et al. 2009). Auch eine weitere Studie zeigte, dass ein Großteil der Patienten mit einer signifikanten Blutdruckreaktion reagierte (Bakris et al. 2012). Diese neue Therapieoption, die die Barorezeptoren einbezieht, ist allerdings noch in der Erprobung und wird zurzeit nur bei Patienten mit einer medikamentenresistenten Hypertonie eingesetzt.

In anderen Studien wurde deutlich, dass eine exogene Vagusnervstimulation neben antikonvulsiven auch antidepressive Auswirkungen hat (Mohr et al. 2011): Über einen Pulsgenerator werden dabei schwache elektrische Signale an den linken Vagusnerv gesendet (Shafique und Dalsing 2006). Der vermutete Einfluss des Vagus auf das limbische System und andere neuroanatomische Strukturen, die mit emotionalen Störungen in Verbindung gebracht werden (Rush et al. 2002), könnte die beobachtete Verbesserung der Stimmung und der Lebensqualität nach sechsmonatiger Vagusnervstimulation erklären (Klinkenberg et al. 2012). Insbesondere bei therapierefraktärer Depression hat sich dieser therapeutische Ansatz als hilfreich erwiesen: 30,5 \% der Patienten zeigten nach 8-wöchiger Vagusnervstimulation eine 50-prozentige Reduktion des Depressivitätsscores (HamiltonDepressionsskala) (Sackeim et al. 2001). Außerdem ergab sich bei der Erprobung als adjuvante Therapie eine Reduktion der antidepressiven Medikation (Shafique und Dalsing 2006). Neben dem arteriellen Blutdruck kann demnach auch die Stimmung durch eine elektrische Stimulation positiv beeinflusst werden. Der beschriebene Einfluss der Barorezeptoren über vagale Afferenzen auf die Stimmung könnte zu der Vermutung führen, dass die neue interventionelle antihypertensive Therapiemethode auch einen Einfluss auf das Wohlbefinden hat. Sollte sich diese bisher noch spekulative Annahme bestätigen, dann würde eine Barorezeptorstimulation möglicherweise nicht nur für therapierefraktäre Patienten in Frage kommen. Patienten mit einer verminderten Lebensqualität unter der Einnahme von Medikamenten und einer geringen Adhärenz könnten möglicherweise ebenfalls von dieser Therapie profitieren. Dazu sollten noch ergänzende Studien stattfinden, die neben der Wirksamkeit der Barorezeptorstimulation hinsichtlich arterieller Blutdruckreaktion auch die Verbesserung des Wohlbefindens untersuchen.

Neben den beschriebenen Hinweisen auf einen Zusammenhang von Lebensqualität und erhöhtem arteriellen Blutdruck haben sich in der vorliegenden Arbeit be- 
reits lange bekannte kardiovaskuläre Risikofaktoren bestätigt: Bei den hypertensiven Jugendlichen waren vermehrt eine Adipositas, ein höherer Alkoholkonsum sowie eine geringere körperliche Fitness zu finden. Daher sollte bereits im Jugendalter durch Präventionsprogramme ein Gesundheitsbewusstsein vermittelt werden, um schädigendes Verhalten erst gar nicht zur Gewohnheit werden zu lassen.

\subsection{Stärken und Schwächen der Arbeit}

Insgesamt handelt es sich bei dem in der KiGGS-Studie untersuchten Kollektiv um eine sehr große und repräsentative Stichprobe, bei der genauestens geplante Befragungen und Untersuchungen durchgeführt wurden. Das Personal wurde geschult und in die Methoden eingewiesen und es fanden regelmäßig Qualitätskontrollen statt. Daher sind anhand des uns zur Verfügung gestellten Datensatzes Auswertungen mit einer suffizienten statistischen Aussagekraft sowie einer Generalisierbarkeit für die deutsche Bevölkerung in der untersuchten Altersgruppe möglich.

Eine Schwäche der KiGGS-Studie betrifft dabei allerdings das Querschnittsdesign. Die Ergebnisse dieser Arbeit können somit nicht als ein kausaler Zusammenhang von erhöhten arteriellen Blutdruckwerten und Lebensqualität interpretiert werden. Das Querschnittsdesign weist zwar auf eine plausible Assoziation beider Faktoren hin, überprüft die Richtung des Zusammenhangs jedoch nicht. Zusätzlich wurden die vorliegenden Analysen zu den Unterschieden zwischen Normo- und Hypertensiven als Post-hoc-Design durchgeführt. Wenn von Unterschieden gesprochen wird, dann lagen lediglich Unterschiede in der Verteilung der Mittelwerte vor. Dennoch ist so die Beschreibung einer unterschiedlichen Verteilung gut möglich. Die Unterschiede waren zwar hinsichtlich der Effektgröße klein, aber zum großen Teil sehr bis höchst signifikant und in sich konsistent, auch bei getrennter Betrachtung der Eigenangaben und der Angaben der Eltern, was auf eine reale und epidemiologisch relevante Assoziation hinweist.

Die Studie war ursprünglich nicht dafür konzipiert, Unterschiede zwischen normotensiven und hypertensiven Jugendlichen herauszufinden. Eine Dichotomisierung in Probanden mit „erhöhtem“ versus „normalem arteriellen Blutdruck“ wurde nach- 
träglich anhand des Blutdruckwerts vorgenommen. Demzufolge ergaben sich zwei unterschiedlich große Kollektive mit einem sehr viel größeren Anteil an normotensiven Jugendlichen. Trotz der ungleichen Verteilung ist die Anzahl von 825 Hypertensiven jedoch noch groß genug, um suffiziente statistische Aussagen treffen zu können.

Bei der Bestimmung des arteriellen Blutdrucks wurde ein oszillometrisches Verfahren durch eine geschulte Person angewendet, obwohl von der NHBPEP Working Group on High Blood Pressure in Children and Adolescents ein auskultatorisches Verfahren empfohlen wird. Außerdem sind die zugrunde liegenden Perzentilkurven anhand auskultatorischer Verfahren bestimmt worden (NHBPEP 2004). Allerdings hat sich gezeigt, dass die oszillometrische Messung weniger untersucherabhängig und damit auch weniger fehleranfällig in der Durchführung ist (Neuhauser und Thamm 2007). Daher ist sie für einen großen Gesundheitssurvey mit vielen unterschiedlichen Untersuchern besser geeignet, um vergleichbare Werte zu erhalten. Die Bedingungen der Untersuchung sind leichter zu standardisieren und Zahlenpräferenz- sowie Untersucherbiasprobleme der auskultatorischen Verfahren lassen sich vermeiden (Gillman und Cook 1995). Zudem sind die Differenzen zwischen den Ergebnissen der auskultatorischen und dem in der KiGGS-Studie verwendeten oszillometrischen Verfahren nur gering. In einer Validierungsstudie zeigte sich, dass die Blutdruckwerte meist weniger als $1 \mathrm{mmHg}$ Abweichung zu den auskultatorischen Werten aufwiesen (Wong et al. 2006). Die Perzentilkurven können daher ohne große Bedenken auch in Bezug auf die vorliegenden Daten verwendet werden. Der Datascope Accutorr Plus ist außerdem ein ausführlich validiertes Messgerät, das von der European Society of Hypertension empfohlen wird (O'Brien et al. 2001). Aufgrund der besseren Praktikabilität im Vergleich zur auskultatorischen Methode wurde daher eine oszillometrische Blutdruckbestimmung vorgezogen.

Zusätzlich hat sich gezeigt, dass die ambulante Blutdruckmessung besser mit den Indizes kardiovaskulärer Dysfunktion korreliert und einen größeren prognostischen Wert hat als standardmäßige Blutdruckmessungen in der Klinik (Ewart und Kolodner 1994). Allerdings war dies in dem großen Setting der KiGGS-Studie nicht umzusetzen.

Es ist aber positiv anzumerken, dass die Bedingungen und Art der Messungen exakt vorgeschrieben und standardisiert waren, nach einem wenig anstrengenden 
Teil der Untersuchung sowie nach fünfminütiger Ruhezeit stattfanden sowie von einem geschulten Mitarbeiter durchgeführt wurden (Neuhauser und Thamm 2007). Die erhobenen Blutdruckdaten wurden publiziert und als neue Referenzwerte für deutsche Kinder und Jugendliche akzeptiert (Neuhauser et al. 2011). Dennoch handelt es sich bei der gewählten Einteilung nicht um eine medizinische Diagnose einer arteriellen Hypertonie, für die mindestens an drei separaten Messzeitpunkten durchschnittlich erhöhte Blutdruckwerte vorliegen müssen (NHBPEP 2004). Die Blutdruckmessung stellt in der vorliegenden Untersuchung lediglich eine Momentaufnahme dar, da nur zu einem Zeitpunkt gemessen wurde. Allerdings wurden in der KiGGS-Studie zwei Messungen vorgenommen, um daraus einen Mittelwert zu bilden. Die Repräsentativität der Werte konnte dadurch zwar etwas verbessert werden, eine Überschätzung der Prävalenz lässt sich aber dennoch nicht ausschließen. Die wiederholte Messung wird empfohlen, da erhöhte Blutdruckwerte bei erneuten Bestimmungen zu anderen Zeitpunkten sinken, zum einen aufgrund eines Gewöhnungseffekts (mit weniger Angst) und zum anderen wegen des $R e$ gression-toward-the-mean-Phänomens (NHBPEP 2004). Allerdings konnte in einer vorherigen Studie gezeigt werden, dass eine Messung zu drei separaten Zeitpunkten zwar zu einer Reduktion des Anteils der als Hypertoniker klassifizierten Personen führt, dafür aber die Zahl der Prähypertoniker ansteigen lässt (McNiece et al. 2007). Diese haben ein erhöhtes Risiko, im Verlauf eine arterielle Hypertonie zu entwickeln (NHBPEP 2004). Selbst wenn die Prävalenz möglicherweise etwas überschätzt wurde, so weist das untersuchte Kollektiv, das als "hypertensiv" bezeichnet wurde, auf jeden Fall ein hohes Risikoprofil für diese Erkrankung auf.

Eine Stärke der vorliegenden Arbeit stellt zudem die genaue Einteilung in normoversus hypertensiv anhand des geschlechts-, größen- und altersadaptierten 95. Blutdruckperzentils dar. Die weit verbreiteten Referenzwerte der NHBPEP Working Group on High Blood Pressure in Children and Adolescents (NHBPEP 2004), die dabei als Grundlage dienten, enthalten Daten normal- und übergewichtiger Jugendlicher und zeigen damit geringfügig höhere Grenzwerte als die zwischenzeitlich publizierten 95. Perzentile aus der KiGGS-Studie, die Übergewichtige nicht einbeziehen (Neuhauser et al. 2011). Eine Studie, die sich auf diese Grenzwerte bezog, gab eine Prävalenz der arteriellen Hypertonie bei übergewichtigen und adipösen Jugendlichen von 35\% an (Flechtner-Mors et al. 2011). Damit ist das von uns definierte hypertensive Kollektiv stärker eingegrenzt und spezifischere 
Aussagen sind möglich. Zudem bezieht sich die hier gewählte Definition des 95. Blutdruckperzentils auf die Grenzwerte, die bisher im internationalen Vergleich in der Regel verwendet wurden. Die Annahme einer korrekten Einteilung in „normotensiv“ und "hypertensiv“ erscheint durch die nachgewiesene Assoziation des erhöhten arteriellen Blutdrucks mit den bekannten Risikofaktoren wie Adipositas, geringer körperlicher Fitness etc. plausibel.

Ein weiterer Kritikpunkt könnte in der Bestimmung der psychosozialen Probleme und Stärken gesehen werden. Hierbei wurden keine Diagnosen psychischer Störungen gestellt, sondern es wurden Anzeichen von Belastungen und Ressourcen in verschiedenen Dimensionen der psychischen Gesundheit erfragt (Hölling et al. 2007 a). Der SDQ ist ein Symptomfragebogen, der aber für klinische Forschung als Screeninginstrument gut geeignet ist, um Eckdaten über die psychische Gesundheit zu erfassen (Klasen et al. 2000; Ravens-Sieberer et al. 2007 b). Bei den der vorliegenden Dissertation zugrunde liegenden Auswertungen zeigten sich zwischen den Subskalen und dem Gesamtproblemwert mittlere bis hohe Korrelationen, was darauf hinweist, dass der SDQ in sich konsistent ist. Auch in vorherigen Studien wurde die interne Konsistenz als zufriedenstellend beurteilt (Cronbachs $\alpha=0,81$ für die Elternangaben sowie 0,74 für die Eigenangaben) (Ravens-Sieberer et al. 2002). Die verwendete deutsche Fassung des SDQ stellt ein gut validiertes und mehrfach psychometrisch getestetes Instrument dar, das sehr gut zur Erfassung von Risikogruppen eingesetzt werden kann (Hölling et al. 2007 a). Demnach kann bei dem Vergleich zwischen den Normo- und Hypertensiven zwar nicht von einer geringeren Prävalenz psychischer Erkrankungen bei letzteren gesprochen werden, es lässt sich allerdings die Tendenz erkennen, dass die Hypertensiven weniger Symptome einer Belastung aufwiesen. Durch den Einsatz des SDQ nicht nur bei den Jugendlichen, sondern zusätzlich bei den Eltern wurde ein umfassenderer Blick ermöglicht. Psychosoziale Auffälligkeiten aus der Sicht verschiedener Personen zu erfassen ist international üblich und gestattet es, potenziell unterschiedliches Verhalten in verschiedenen Lebenssituationen zu erkennen (RavensSieberer et al. 2002). Zwischen den Eltern- und Eigenangaben zeigten sich hier jedoch nur geringe Korrelationen. Dies könnte ein Hinweis auf die zunehmende Abgrenzung der Jugendlichen während der Pubertät sein und verdeutlichen, dass Eltern und Kinder in diesem Alter oft unterschiedliche Wahrnehmungen ihrer Le- 
benssituation haben. Umso bemerkenswerter ist es, dass sowohl Eigenangaben als auch Elternangaben in fast identischer Weise mit dem Blutdruck assoziiert waren.

Ein Vergleich der gesundheitsbezogenen Lebensqualität zwischen verschiedenen Studien gestaltet sich aufgrund der vielen unterschiedlichen Erfassungsmethoden schwierig. Da die bisherigen Studien bezüglich des Zusammenhangs von erhöhten arteriellen Blutdruckwerten und Lebensqualität vorwiegend an Erwachsenen stattfanden, wurden hier andere Instrumente verwendet als der KINDL-R. Allerdings sollten Bewertungen der gesundheitsbezogenen Lebensqualität von Erwachsenen nicht mit denen von Jugendlichen gleichgesetzt werden, da beide Gruppen unterschiedliche Kriterien für die Beurteilung heranziehen (RavensSieberer 2000). Die Unterschiede zwischen den Studien an Erwachsenen und Jugendlichen wurden möglicherweise zusätzlich durch diesen Aspekt beeinflusst. Aufgrund der Tatsache, dass Jugendliche andere Dimensionen für die selbst wahrgenommene Lebensqualität als relevant ansehen als Erwachsene, war die Entwicklung eines Fragebogens, der speziell für das Kindes- und Jugendalter entwickelt und validiert wurde, sinnvoll (Schumacher et al. 2003). Mit dem KINDL$\mathrm{R}$ liegt ein original deutschsprachiges und international standardisiertes Instrument vor (Schumacher et al. 2003), das Schwierigkeiten durch Übersetzungen umgeht. Die Hauptkomponenten für die Lebensqualität im Jugendalter sind das Selbstwertgefühl, die Beziehung zu Familie und Freunden sowie das schulische Wohlbefinden (Rajmil et al. 2004), die vollständig mit dem KINDL-R Fragebogen erfasst wurden. Die Lebensqualität wurde demnach in der KiGGS-Studie umfassend und altersangepasst erhoben. Wie bereits bei den psychosozialen Problemen gab es auch bei der Beurteilung der Lebensqualität neben der Selbstauskunft auch Elternangaben, die untereinander nur eine geringe Korrelation aufwiesen. Allerdings sollten die Elternangaben nicht als Überprüfung der Antworten der Jugendlichen interpretiert werden, sondern viel mehr als ergänzende Informationsquelle (Ravens-Sieberer 2000). Interessanterweise waren beim Vergleich zwischen den Normo- und Hypertensiven die gleichen Subskalen bei Eigen- und Fremdangaben signifikant unterschiedlich, was die gefundenen Trends unterstreicht. Es hat sich gezeigt, dass die einzelnen Subskalen stark miteinander zusammenhängen (Bullinger et al. 1994), sodass noch eine stärkere Trennschärfe wünschenswert wäre. Möglicherweise sind die verschiedenen Lebensqualitätsbereiche im Ju- 
gendalter aber auch stark miteinander verkettet (Bullinger et al. 1994). Bei der psychometrischen Testung zeigte der KINDL-R eine hohe Akzeptanz sowie eine hohe Reliabilität in den meisten Skalen und untersuchten Kollektiven (Bullinger et al. 1994; Ravens-Sieberer und Bullinger 1998; Ravens-Sieberer 2000; RavensSieberer et al. 2002). Die interne Konsistenz ist wie beim SDQ als zufriedenstellend zu bezeichnen (Cronbachs $\alpha=0,85$ für die Eigenangaben und 0,82 für die Elternangaben) (Ravens-Sieberer et al. 2002). Zusätzlich erwies er sich als sehr praktikabel und ist damit für den Einsatz in Surveys gut geeignet (Ravens-Sieberer et al. 2002; Rajmil et al. 2004).

In der multivariaten Testung zeigten sich die Ergebnisse in den Bereichen der Lebensqualität und der psychosozialen Probleme zwar weiterhin als signifikant, allerdings war die Modellanpassungsgüte bei den verschiedenen Modellen eher gering, sodass die gefundene Assoziation auch kritisch gesehen werden könnte. Die umgekehrte Betrachtung mit der Lebensqualität bzw. der psychischen Gesundheit als abhängigen Variablen bestätigte den erhöhten arteriellen Blutdruck aber ebenfalls als signifikante Determinante, was das Konstrukt eines Zusammenhangs von arteriellem Blutdruck und Wohlbefinden unterstützt. Die hierbei gefundenen Effektgrößen waren zwar gering, was aber aufgrund der nur geringen Spannbreite der Blutdruckwerte, der Komplexität des psychosozialen Befindens sowie der Heterogenität der Stichprobe zu erwarten war. Außerdem lagen die gefundenen Effektstärken im Bereich anderer bekannter Prädiktoren des Wohlbefindens wie beispielsweise des Geschlechts und des BMIs.

Ein weiterer möglicher Kritikpunkt ergibt sich bei der Erfassung der Schutzfaktoren. Dabei wurden die Bereiche soziale, personale und familiäre Ressourcen durch Items bestimmt, die aus verschiedenen Fragebögen stammen. Da diese jedoch nur teilweise in die KiGGS-Studie übernommen wurden, könnte eine geringere Validität vermutet werden. Im Pretest vor der eigentlichen KiGGS-Studie hat sich allerdings gezeigt, dass die neue Zusammensetzung der verschiedenen Items als Determinante von psychischer Gesundheit angesehen werden kann und zudem noch eine gute Akzeptanz sowie eine gute interne Konsistenz aufweist (Ravens-Sieberer et al. 2002). Bei der vorliegenden Untersuchung waren jedoch trotz unterschiedlicher Lebensqualität keine Unterschiede bei den Ressourcen zwischen den Gruppen mit normalem und erhöhtem Blutdruck zu finden. Dies könnte einerseits zu der Vermutung führen, dass die neu gewählte Zusammenset- 
zung der Items aus verschiedenen Skalen die Schutzfaktoren möglicherweise doch nicht genau erfasst. Andererseits könnten die fehlenden Unterschiede zwischen normo- und hypertensiven Jugendlichen auch darauf hinweisen, dass äußere Schutzfaktoren wie familiäre und soziale Ressourcen durch den Blutdruck erwartungsgemäß unbeeinflusst bleiben - im Gegensatz zu inneren Schutzfaktoren wie zum Beispiel das durch den KINDL-R erfasste Selbstwertgefühl.

Bei der ergänzenden Erfassung des Freizeit- und Risikoverhaltens sowie des allgemeinen Befindens lässt sich kritisieren, dass diese Variablen nur semiquantitative Antwortmöglichkeiten aufwiesen. Dies schränkt die Qualität der Daten etwas ein. Dennoch haben sich bei den Untersuchungen die meisten der bekannten Risikofaktoren bestätigt, sodass von einer ausreichenden Datenerfassung ausgegangen werden kann. Als weiterer Kritikpunkt wäre die Beurteilung des Schuldurchlaufs anhand einer einzigen Variablen zu nennen. Es wurde nur erfasst, ob Klassen wiederholt wurden oder ob ein regulärer Schuldurchlauf stattgefunden hatte. Ob die hypertensiven Jugendlichen insgesamt wirklich bessere Schulleistungen aufwiesen, kann demnach nicht bestätigt werden. Allerdings weist die Tatsache, dass weniger Hypertensive Klassen wiederholten, darauf hin. 


\subsection{Schlussfolgerung}

Eine hohe Prävalenz erhöhter arterieller Blutdruckwerte im Jugendalter verdeutlicht die Relevanz und die Bedeutung von Präventionsmaßnahmen bereits in jungen Jahren. In den Auswertungen bestätigten sich bekannte Risikofaktoren von Erwachsenen zumindest korrelativ auch bei hypertonen Jugendlichen, was einen Ansatz für zukünftige Interventionsmöglichkeiten darstellt.

Insgesamt sind die erhobenen Unterschiede zwischen Hyper- und Normotensiven zwar nur gering, dafür größtenteils aber höchst signifikant und sowohl im Bereich der psychischen Gesundheit als auch der Lebensqualität zu finden. Die große Anzahl der untersuchten Teilnehmer weist auf einen epidemiologisch relevanten Zusammenhang eines erhöhten arteriellen Blutdrucks und einer besseren Lebensqualität hin. Der Vorteil dieser Untersuchung, ein „unverfälschtes“ Kollektiv ohne Medikamentennebenwirkungen, Folgeerkrankungen oder Beeinträchtigungen durch das Wissen, erkrankt zu sein, ermöglicht eine unbeeinflusste Assoziation des arteriellen Blutdrucks und des Befindens zu erfassen. So ergaben sich erstmals Hinweise, dass bei Jugendlichen ohne die zuvor genannten Einflussfaktoren eine positive Korrelation von Lebensqualität und erhöhtem arteriellen Blutdruck vorliegen könnte. Damit konnte die bereits 1988 an Erwachsenen nachgewiesene inverse Assoziation erhöhter Blutdruckwerte und subjektiver Probleme (Winkleby et al. 1988) an einem jugendlichen Kollektiv bestätigt werden. Dies verweist auf einen möglichen stressreduzierenden Effekt des erhöhten Blutdrucks im Anfangsstadium der arteriellen Hypertonie, wenn ein vaskuläres Remodelling noch nicht eingetreten ist. Eine mögliche Erklärung für die positive Korrelation stellt dabei der Ansatz der erlernten arteriellen Hypertonie dar, der bisher noch nicht ausreichend erforscht und bestätigt wurde. Obwohl sich eindeutig die Tendenz eines besseren Wohlbefindens feststellen ließ, kann aufgrund des Querschnittsdesigns nicht von einem kausalen Zusammenhang ausgegangen werden. Deshalb lässt sich auch nicht abschließend sagen, wodurch die erhöhten Blutdruckwerte bei den Jugendlichen zustande gekommen sind. Sowohl die Theorie der "learned hypertension" als auch die Theorie der Affektrepression kommen als Erklärungsmodelle in Betracht. Ebenfalls denkbar wäre, dass die Jugendlichen einerseits allein durch den besseren Schulerfolg und die höhere soziale Angepasstheit mehr Zufriedenheit aufwiesen, aber andererseits aufgrund des damit verbundenen größeren Stresses erhöh- 
te Blutdruckwerte entwickelten. Diese drei Erklärungsansätze schließen sich nicht gegenseitig aus. Vielmehr sollten sie als Ansatzpunkt für weitere Studien dienen. Zukünftige Untersuchungen sollten die Ergebnisse dieser Arbeit in einer longitudinal organisierten Studie untersuchen, um den vermuteten kausalen Zusammenhang nachzuweisen. Dabei sollten Probanden, die lediglich erhöhte Blutdruckwerte aufweisen, im Mittelpunkt stehen, um zu überprüfen, ob wirklich die Abwesenheit von Komorbidität eine Grundvoraussetzung für den Zusammenhang von erhöhtem Blutdruck und besserer Lebensqualität ist und welchen Einfluss Medikamente und krankheitsbezogene Ängste dabei spielen. Ließen sich die Ergebnisse der vorliegenden Arbeit bestätigen, dann könnten sich daraus neue Interventionsmöglichkeiten für das stetig wachsende Problem des erhöhten arteriellen Blutdrucks schon im Jugendalter ergeben. 


\section{Zusammenfassung}

Erhöhter arterieller Blutdruck gewinnt im Jugendalter zunehmend an Bedeutung. Aufgrund der hohen Prävalenz sowie der langfristigen medizinischen Konsequenzen stellt er für die Zukunft eine große Belastung für die heranwachsende Generation und das Gesundheitswesen dar. Dies verweist auf die Notwendigkeit, die zugrunde liegenden Ursachen besser zu verstehen. Daher sollten insbesondere die kardiovaskulären und psychosomatischen Korrelate bei Jugendlichen genauer betrachtet werden. Neben der Darstellung von Unterschieden in Freizeitverhalten und Lebensgewohnheiten sollte mit der vorliegenden Untersuchung die Hypothese überprüft werden, dass bereits im Jugendalter ein erhöhter Blutdruck mit reduziertem Wohlbefinden assoziiert ist.

Um möglichst umfassende Ergebnisse zu erhalten und epidemiologisch hochwertige Aussagen treffen zu können, wurde die Analyse anhand von Daten des großen Kinder- und Jugendgesundheitssurveys (KiGGS) durchgeführt. Dabei wurde eine für Deutschland repräsentative und umfangreiche Stichprobe von insgesamt 7697 Studienteilnehmern im Alter von 11 bis 17 Jahren untersucht.

Der Blutdruck wurde oszillometrisch von einem eingewiesenen und erfahrenen Untersucher bestimmt. Die Einteilung der Jugendlichen in hyper- und normotensiv erfolgte entsprechend dem alters-, geschlechts- und größenadjustierten 95. Blutdruckperzentil gemäß den Vorgaben für das Kindes- und Jugendalter. Die Lebensqualität und die psychische Gesundheit wurden mithilfe von gut validierten Erhebungsinstrumenten, dem KINDL-R und der deutschen Version des SDQ, erhoben.

10,7 \% (825) der Jugendlichen wiesen einen erhöhten arteriellen Blutdruck auf mit einem größeren Anteil an männlichen Teilnehmern (61,3\%).

Bekannte kardiovaskuläre Risikofaktoren der Erwachsenen stellten sich als ebenfalls relevant für das Jugendalter heraus. So zeigten die Hypertensiven eine höhere Wahrscheinlichkeit, adipös zu sein, und berichteten häufiger von einem für die Gesundheit ungünstigen Verhalten wie einem ausgeprägten Computer- und Alkoholkonsum und einer schlechten körperlichen Fitness. Andererseits gaben sie inte- 
ressanterweise häufiger schulischen Erfolg und geringere Hyperaktivitätsprobleme an.

Entgegen den anfänglichen Erwartungen konnte eine Assoziation eines erhöhten arteriellen Blutdrucks mit einer besseren Lebensqualität bzw. geringeren psychosozialen Problemen festgestellt werden. Bei der Lebensqualität erwiesen sich die Dimensionen „Familie“, „Selbstwert" und „körperliches Wohlbefinden“ sowie der übergreifende Messwert bei Eigen- und Elternangaben als signifikant unterschiedlich. Bei den psychosozialen Schwierigkeiten ergaben sich für die Subskala „Hyperaktivität“ bei Eltern- und Eigenangaben, für die Bereiche „emotionale Probleme" und „Verhaltensprobleme" sowie den Gesamtproblemwert nur für die Elternangaben signifikante Unterschiede. Diese positive Assoziation eines erhöhten arteriellen Blutdrucks und besserer Lebensqualität bzw. psychischer Gesundheit zeigte sich sowohl in univariaten als auch in multivariaten Analysen. In letzteren war auch der selbst berichtete Gesamtproblemwert signifikant mit dem Blutdruck assoziiert. Ergänzend bestätigte sich der erhöhte arterielle Blutdruck als Prädiktor für eine bessere Lebensqualität mit weniger psychosozialen Problemen auch in der umgekehrten Betrachtung mit dem Wohlbefinden als abhängige Variable.

Die vorliegende Beobachtung eines Zusammenhangs von erhöhtem arteriellen Blutdruck und besserem Wohlbefinden steht im Kontrast zu der Mehrzahl der vorhergehenden Studien an Erwachsenen. Dies verweist auf das Vorhandensein altersabhängiger Faktoren, insbesondere die Abwesenheit von Komorbiditäten, Folgeerkrankungen und Medikamentennebenwirkungen sowie das fehlende Bewusstsein, an einer arteriellen Hypertonie erkrankt zu sein. Im Gegensatz zu Erwachsenen konnten hinsichtlich der zuvor genannten Faktoren keine Unterschiede zwischen hyper- und normotensiven Jugendlichen festgestellt werden.

Allerdings stimmen die Ergebnisse dieser Arbeit mit vorherigen Studien überein, die zeigten, dass ein Anstieg des Blutdrucks entspannende und stresslindernde Effekte über vagale Afferenzen hat. Dieser Nebeneffekt bei der Aktivierung von Barorezeptoren könnte als angenehm und belohnend wahrgenommen werden und so von einer zunächst kurzfristigen Blutdruckerhöhung zu einer langfristig „konditionierten“ oder „erlernten“ arteriellen Hypertonie führen. Möglicherweise ist diese stress- und angstlindernde Wirkung im Rahmen eines erhöhten arteriellen Blutdrucks nur in den frühen Stadien der Erkrankung vorhanden, solange es noch 
nicht zu einem vaskulären Remodelling gekommen ist. Möglicherweise wird ein solcher Effekt aber auch bei Erwachsenen lediglich durch gegenläufige Effekte der beschriebenen Konsequenzen einer diagnostizierten Hypertonie verdeckt. Dies könnte für den Unterschied in der Lebensqualität zwischen hypertensiven Jugendlichen und Erwachsenen verantwortlich sein. Ein anderer Erklärungsansatz wäre die Theorie der Affektrepression, wonach durch eine Verdrängung negativer Emotionen einerseits zwar ein besseres Wohlbefinden, andererseits jedoch auch ein erhöhter arterieller Blutdruck bedingt sein könnte.

Zukünftige Studien sollten den in dieser Arbeit gefundenen Zusammenhang unter Berücksichtigung der oben genannten altersabhängigen Einflussfaktoren auf Kausalität überprüfen. Sollte sich der Zusammenhang von arterieller Hypertonie und besserer Lebensqualität infolge einer Abschwächung von psychosozialem Stress und Angst über vagale Afferenzen von Barorezeptoren bestätigen, dann würde dies die Hypothese der „erlernten arteriellen Hypertonie“ unterstützen. Möglicherweise könnten sich dann neue kausale Therapieoptionen für die essentielle arterielle Hypertonie ableiten lassen. Gleiches würde auch gelten, wenn sich die Theorie der Affektrepression bestätigen sollte. 


\section{Anhang: Tabellen I-XIII}

Tab. I Kontrollvariablen und Confounder mit OR \& Signifikanz im univariaten und multivariaten Modell am Bsp. des körperlichen Wohlbefindens (Eigenangaben)

\begin{tabular}{|c|c|c|c|c|c|c|}
\hline \multirow{2}{*}{ KINDL-R } & \multicolumn{2}{|c|}{ univariat } & \multicolumn{2}{|c|}{$\begin{array}{l}\text { multivariat: } \\
\text { Modell } 1\end{array}$} & \multicolumn{2}{|c|}{$\begin{array}{l}\text { multivariat: } \\
\text { Modell 1b }\end{array}$} \\
\hline & OR & Signifikanz & OR & Signifikanz & $O R$ & Signifikanz \\
\hline $\begin{array}{c}\text { Körperliches WB } \\
\text { (Eigenangaben) }\end{array}$ & 1,009 & $p<0,001$ & 1,012 & $p<0,001$ & 1,014 & $p<0,001$ \\
\hline Alter & 1,087 & $p<0,001$ & 1,024 & $\varnothing$ sign. & 1,005 & $\varnothing$ sign. \\
\hline Geschlecht & 1,580 & $p<0,001$ & 1,512 & $p<0,001$ & 1,504 & $p<0,001$ \\
\hline BMI & 1,153 & $p<0,001$ & 1,164 & $p<0,001$ & 1,164 & $p<0,001$ \\
\hline \multicolumn{7}{|l|}{ Sozialer Status } \\
\hline mittel vs. hoch & 1,019 & $\varnothing$ sign. & 0,925 & $\varnothing$ sign. & 0,896 & $\varnothing$ sign. \\
\hline niedrig vs. hoch & 1,059 & $\varnothing$ sign. & 0,761 & $p<0,05$ & 0,778 & $p<0,05$ \\
\hline Einschränkungen & 1,500 & $p<0,01$ & 1,108 & $\varnothing$ sign. & 1,060 & $\varnothing$ sign. \\
\hline \multicolumn{7}{|l|}{ Schuldurchlauf } \\
\hline $\begin{array}{l}\text { übersprungen vs. } \\
\text { regulär }\end{array}$ & 0,333 & $p=0,067$ & 0,397 & $\varnothing$ sign. & 0,430 & $\varnothing$ sign. \\
\hline $\begin{array}{l}\text { wiederholt vs. regu- } \\
\text { lär }\end{array}$ & 0,773 & $p<0,05$ & 0,604 & $p<0,001$ & 0,553 & $p<0,001$ \\
\hline \multicolumn{7}{|l|}{$\begin{array}{c}\text { Körperliche Leis- } \\
\text { tungsfähigkeit }\end{array}$} \\
\hline gut vs. sehr gut & 1,258 & $p<0,05$ & 1,131 & $\varnothing$ sign. & 1,170 & $\varnothing$ sign. \\
\hline mittel vs. sehr gut & 1,461 & $p \leq 0,001$ & 1,087 & $\varnothing$ sign. & 1,249 & $p=0,096$ \\
\hline $\begin{array}{l}\text { nicht besonders gut } \\
\text { vs. sehr gut }\end{array}$ & 1,712 & $p \leq 0,001$ & 0,994 & $\varnothing$ sign. & 1,193 & $\varnothing$ sign. \\
\hline $\begin{array}{l}\text { gar nicht gut vs. } \\
\text { sehr gut }\end{array}$ & 1,189 & $\varnothing$ sign. & 0,333 & $p=0,057$ & 0,398 & $p=0,079$ \\
\hline
\end{tabular}




\begin{tabular}{|c|c|c|c|c|c|c|}
\hline TV/Video & & & & & & \\
\hline $0,5 \mathrm{~h}$ vs. gar nicht & 1,345 & $\varnothing$ sign. & 1,596 & $p=0,089$ & 1,554 & $\varnothing$ sign. \\
\hline 1-2h vs. gar nicht & 1,858 & $p<0,01$ & 1,998 & $p<0,01$ & 1,984 & $p<0,05$ \\
\hline 3-4h vs. gar nicht & 2,007 & $p<0,01$ & 2,095 & $p<0,01$ & 2,197 & $p<0,01$ \\
\hline$>4 \mathrm{~h}$ vs. gar nicht & 1,801 & $p<0,05$ & 1,506 & $\varnothing$ sign. & 1,581 & $\varnothing$ sign. \\
\hline \multicolumn{7}{|l|}{ Computer } \\
\hline $0,5 \mathrm{~h}$ vs. gar nicht & 0,928 & $\varnothing$ sign. & 0,900 & $\varnothing$ sign. & 0,897 & $\varnothing$ sign. \\
\hline 1-2h vs. gar nicht & 1,010 & $\varnothing$ sign. & 0,851 & $\varnothing$ sign. & 0,828 & $\varnothing$ sign. \\
\hline 3-4h vs. gar nicht & 1,211 & $\varnothing$ sign. & 0,859 & $\varnothing$ sign. & 0,871 & $\varnothing$ sign. \\
\hline$>4 \mathrm{~h}$ vs. gar nicht & 1,889 & $p<0,001$ & 1,274 & $\varnothing$ sign. & 1,394 & $p=0,089$ \\
\hline \multicolumn{7}{|l|}{ Bier } \\
\hline $\begin{array}{l}>=1 \mathrm{Glas} / \mathrm{d} \\
\text { vs. gar nicht }\end{array}$ & 2,486 & $p<0,01$ & 1,800 & $p=0,070$ & 1,898 & $p=0,070$ \\
\hline $\begin{array}{l}\text { 5-6 Gläser/Woche } \\
\text { vs. gar nicht }\end{array}$ & 1,519 & $p<0,05$ & 1,203 & $\varnothing$ sign. & 1,253 & $\varnothing$ sign. \\
\hline $\begin{array}{l}\text { 2-4 Gläser/Woche } \\
\text { vs. gar nicht }\end{array}$ & 1,663 & $p<0,001$ & 1,408 & $\mathrm{p}<0,05$ & 1,523 & $p<0,01$ \\
\hline $\begin{array}{l}1 \text { Glas/Woche } \\
\text { vs. gar nicht }\end{array}$ & 1,222 & $\varnothing$ sign. & 1,062 & $\varnothing$ sign. & 1,020 & $\varnothing$ sign. \\
\hline $\begin{array}{l}\text { 1-3 Gläser/Monat } \\
\text { vs. gar nicht }\end{array}$ & 1,336 & $p<0,05$ & 1,314 & $\mathrm{p}<0,05$ & 1,309 & $p=0,053$ \\
\hline $\begin{array}{l}<1 \text { Glas/Monat } \\
\text { vs. gar nicht }\end{array}$ & 0,726 & $p<0,05$ & 0,708 & $p<0,05$ & 0,758 & $p=0,053$ \\
\hline $\begin{array}{c}\text { Anpassungsgüte } \\
-2 L L\end{array}$ & & & \multicolumn{2}{|c|}{4470,125} & \multicolumn{2}{|c|}{4291,786} \\
\hline
\end{tabular}

Tab. II Kontrollvariablen und Confounder mit $O R$ \& Signifikanz im univariaten und multivariaten Modell am Bsp. Selbstwert (Eigenangaben)

\begin{tabular}{|c|c|c|c|c|c|c|}
\hline \multirow{2}{*}{ KINDL-R } & \multicolumn{2}{|c|}{ univariat } & \multicolumn{2}{c|}{$\begin{array}{c}\text { multivariat: } \\
\text { Modell 1 }\end{array}$} & \multicolumn{2}{c|}{$\begin{array}{c}\text { multivariat: } \\
\text { Modell 1b }\end{array}$} \\
\cline { 2 - 7 } & OR & & OR & Signifikanz & OR & Signifikanz \\
\hline $\begin{array}{c}\text { Selbstwert } \\
\text { (Eigenangaben) }\end{array}$ & 1,005 & $\mathrm{p}<0,05$ & 1,005 & $\mathrm{p}<0,05$ & 1,005 & $\mathrm{p}<0,05$ \\
\hline Alter & 1,087 & $\mathrm{p}<0,001$ & 1,000 & $\varnothing$ sign. & 0,982 & $\varnothing$ sign. \\
\hline Geschlecht & 1,580 & $\mathrm{p}<0,001$ & 1,627 & $\mathrm{p}<0,001$ & 1,612 & $\mathrm{p}<0,001$ \\
\hline BMI & 1,153 & $\mathrm{p}<0,001$ & 1,163 & $\mathrm{p}<0,001$ & 1,166 & $\mathrm{p}<0,001$ \\
\hline
\end{tabular}




\begin{tabular}{|c|c|c|c|c|c|c|}
\hline \multicolumn{7}{|l|}{ Sozialer Status } \\
\hline mittel vs. hoch & 1,019 & $\varnothing$ sign. & 0,910 & $\varnothing$ sign. & 0,882 & $\varnothing$ sign. \\
\hline niedrig vs. hoch & 1,059 & $\varnothing$ sign. & 0,761 & $p<0,05$ & 0,770 & $p<0,05$ \\
\hline Einschränkungen & 1,500 & $p<0,01$ & 1,108 & $\varnothing$ sign. & 1,106 & $\varnothing$ sign. \\
\hline \multicolumn{7}{|l|}{ Schuldurchlauf } \\
\hline $\begin{array}{l}\text { übersprungen vs. } \\
\text { regulär }\end{array}$ & 0,333 & $p=0,067$ & 0,397 & $\varnothing$ sign. & 0,447 & $\varnothing$ sign. \\
\hline $\begin{array}{l}\text { wiederholt vs. regu- } \\
\text { lär }\end{array}$ & 0,773 & $p<0,05$ & 0,604 & $p<0,001$ & 0,549 & $p<0,001$ \\
\hline \multicolumn{7}{|l|}{$\begin{array}{l}\text { Körperliche Leis- } \\
\text { tungsfähigkeit }\end{array}$} \\
\hline gut vs. sehr gut & 1,258 & $p<0,05$ & 1,131 & $\varnothing$ sign. & 1,108 & $\varnothing$ sign. \\
\hline mittel vs. sehr gut & 1,461 & $p \leq 0,001$ & 1,087 & $\varnothing$ sign. & 1,123 & $\varnothing$ sign. \\
\hline $\begin{array}{l}\text { nicht besonders gut } \\
\text { vs. sehr gut }\end{array}$ & 1,712 & $p \leq 0,001$ & 0,994 & $\varnothing$ sign. & 1,032 & $\varnothing$ sign. \\
\hline $\begin{array}{l}\text { gar nicht gut vs. } \\
\text { sehr gut }\end{array}$ & 1,189 & $\varnothing$ sign. & 0,333 & $p=0,057$ & 0,341 & $p=0,067$ \\
\hline \multicolumn{7}{|l|}{ TV/Video } \\
\hline $0,5 \mathrm{~h}$ vs. gar nicht & 1,345 & $\varnothing$ sign. & 1,596 & $p=0,089$ & 1,511 & $\varnothing$ sign. \\
\hline $1-2 \mathrm{~h}$ vs. gar nicht & 1,858 & $p<0,01$ & 1,998 & $p<0,01$ & 1,928 & $p<0,05$ \\
\hline 3-4h vs. gar nicht & 2,007 & $p<0,01$ & 2,095 & $p<0,01$ & 2,007 & $p<0,01$ \\
\hline$>4 \mathrm{~h}$ vs. gar nicht & 1,801 & $p<0,05$ & 1,506 & $\varnothing$ sign. & 1,484 & $\varnothing$ sign. \\
\hline \multicolumn{7}{|l|}{ Computer } \\
\hline $0,5 \mathrm{~h}$ vs. gar nicht & 0,928 & $\varnothing$ sign. & 0,900 & $\varnothing$ sign. & 0,874 & $\varnothing$ sign. \\
\hline $1-2 \mathrm{~h}$ vs. gar nicht & 1,010 & $\varnothing$ sign. & 0,851 & $\varnothing$ sign. & 0,801 & $p=0,057$ \\
\hline 3-4h vs. gar nicht & 1,211 & $\varnothing$ sign. & 0,859 & $\varnothing$ sign. & 0,830 & $\varnothing$ sign. \\
\hline$>4 \mathrm{~h}$ vs. gar nicht & 1,889 & $p<0,001$ & 1,274 & $\varnothing$ sign. & 1,365 & $\varnothing$ sign. \\
\hline \multicolumn{7}{|l|}{ Bier } \\
\hline $\begin{array}{l}>=1 \text { Glas/d } \\
\text { vs. gar nicht }\end{array}$ & 2,486 & $p<0,01$ & 1,800 & $p=0,070$ & 1,871 & $p=0,079$ \\
\hline $\begin{array}{l}\text { 5-6 Gläser/Woche } \\
\text { vs. gar nicht }\end{array}$ & 1,519 & $p<0,05$ & 1,203 & $\varnothing$ sign. & 1,248 & $\varnothing$ sign. \\
\hline $\begin{array}{l}\text { 2-4 Gläser/Woche } \\
\text { vs. gar nicht }\end{array}$ & 1,663 & $p<0,001$ & 1,408 & $p<0,05$ & 1,550 & $p<0,01$ \\
\hline $\begin{array}{l}1 \text { Glas/Woche } \\
\text { vs. gar nicht }\end{array}$ & 1,222 & $\varnothing$ sign. & 1,062 & $\varnothing$ sign. & 1,015 & $\varnothing$ sign. \\
\hline $\begin{array}{l}\text { 1-3 Gläser/Monat } \\
\text { vs. gar nicht }\end{array}$ & 1,336 & $p<0,05$ & 1,314 & $p<0,05$ & 1,356 & $p<0,05$ \\
\hline $\begin{array}{l}<1 \text { Glas/Monat } \\
\text { vs. gar nicht }\end{array}$ & 0,726 & $p<0,05$ & 0,708 & $p<0,05$ & 0,768 & $p=0,065$ \\
\hline $\begin{array}{c}\text { Anpassungsgüte } \\
-2 \mathrm{LL}\end{array}$ & & & \multicolumn{2}{|c|}{4470,125} & \multicolumn{2}{|c|}{4302,015} \\
\hline
\end{tabular}


Tab. III Kontrollvariablen und Confounder mit OR \& Signifikanz im bivariaten und multivariaten Modell am Bsp. Familie (Eigenangaben)

\begin{tabular}{|c|c|c|c|c|c|c|}
\hline \multirow{2}{*}{ KINDL-R } & \multicolumn{2}{|c|}{ univariat } & \multicolumn{2}{|c|}{$\begin{array}{c}\text { multivariat: } \\
\text { Modell } 1\end{array}$} & \multicolumn{2}{|c|}{$\begin{array}{l}\text { multivariat: } \\
\text { Modell 1b }\end{array}$} \\
\hline & $O R$ & Signifikanz & OR & Signifikanz & OR & Signifikanz \\
\hline $\begin{array}{c}\text { Familie } \\
\text { (Eigenangaben) }\end{array}$ & 1,005 & $p<0,05$ & 1,007 & $p<0,01$ & 1,009 & $p<0,01$ \\
\hline Alter & 1,087 & $p<0,001$ & 1,013 & $\varnothing$ sign. & 0,993 & $\varnothing$ sign. \\
\hline Geschlecht & 1,580 & $p<0,001$ & 1,683 & $p<0,001$ & 1,647 & $p<0,001$ \\
\hline$B M I$ & 1,153 & $p<0,001$ & 1,160 & $p<0,001$ & 1,163 & $p<0,001$ \\
\hline \multicolumn{7}{|l|}{ Sozialer Status } \\
\hline mittel vs. hoch & 1,019 & $\varnothing$ sign. & 0,909 & $\varnothing$ sign. & 0,885 & $\varnothing$ sign. \\
\hline niedrig vs. hoch & 1,059 & $\varnothing$ sign. & 0,760 & $p<0,05$ & 0,779 & $p<0,05$ \\
\hline Einschränkungen & 1,500 & $p<0,01$ & 1,108 & $\varnothing$ sign. & 1,111 & $\varnothing$ sign. \\
\hline \multicolumn{7}{|l|}{ Schuldurchlauf } \\
\hline $\begin{array}{c}\text { übersprungen vs. } \\
\text { regulär }\end{array}$ & 0,333 & $p=0,067$ & 0,397 & $\varnothing$ sign. & 0,427 & $\varnothing$ sign. \\
\hline $\begin{array}{l}\text { wiederholt vs. regu- } \\
\text { lär }\end{array}$ & 0,773 & $p<0,05$ & 0,604 & $p<0,001$ & 0,552 & $p<0,001$ \\
\hline \multicolumn{7}{|l|}{$\begin{array}{c}\text { Körperliche Leis- } \\
\text { tungsfähigkeit }\end{array}$} \\
\hline gut vs. sehr gut & 1,258 & $p<0,05$ & 1,131 & $\varnothing$ sign. & 1,101 & $\varnothing$ sign. \\
\hline mittel vs. sehr gut & 1,461 & $p \leq 0,001$ & 1,087 & $\varnothing$ sign. & 1,111 & $\varnothing$ sign. \\
\hline $\begin{array}{l}\text { nicht besonders gut } \\
\text { vs. sehr gut }\end{array}$ & 1,712 & $p \leq 0,001$ & 0,994 & $\varnothing$ sign. & 1,010 & $\varnothing$ sign. \\
\hline $\begin{array}{l}\text { gar nicht gut vs. } \\
\text { sehr gut }\end{array}$ & 1,189 & $\varnothing$ sign. & 0,333 & $p=0,057$ & 0,353 & $p=0,077$ \\
\hline \multicolumn{7}{|l|}{ TV/Video } \\
\hline $0,5 \mathrm{~h}$ vs. gar nicht & 1,345 & $\varnothing$ sign. & 1,596 & $p=0,089$ & 1,560 & $\varnothing$ sign. \\
\hline $1-2 \mathrm{~h}$ vs. gar nicht & 1,858 & $p<0,01$ & 1,998 & $p<0,01$ & 1,989 & $p<0,05$ \\
\hline $3-4$ h vs. gar nicht & 2,007 & $p<0,01$ & 2,095 & $p<0,01$ & 2,159 & $p<0,01$ \\
\hline$>4 \mathrm{~h}$ vs. gar nicht & 1,801 & $p<0,05$ & 1,506 & $\varnothing$ sign. & 1,435 & $\varnothing$ sign. \\
\hline \multicolumn{7}{|l|}{ Computer } \\
\hline $0,5 \mathrm{~h}$ vs. gar nicht & 0,928 & $\varnothing$ sign. & 0,900 & $\varnothing$ sign. & 0,903 & $\varnothing$ sign. \\
\hline $1-2 \mathrm{~h}$ vs. gar nicht & 1,010 & $\varnothing$ sign. & 0,851 & $\varnothing$ sign. & 0,825 & $p=0,099$ \\
\hline 3-4h vs. gar nicht & 1,211 & $\varnothing$ sign. & 0,859 & $\varnothing$ sign. & 0,858 & $\varnothing$ sign. \\
\hline$>4 \mathrm{~h}$ vs. gar nicht & 1,889 & $p<0,001$ & 1,274 & $\varnothing$ sign. & 1,463 & $p<0,05$ \\
\hline
\end{tabular}




\begin{tabular}{|c|c|c|c|c|c|c|}
\hline Bier & \multicolumn{7}{|c|}{$\begin{array}{c}\text { >= 1 Glas/d } \\
\text { vs. gar nicht }\end{array}$} & 2,486 & $\mathrm{p}<0,01$ & 1,800 & $\mathrm{p}=0,070$ & 1,930 & $\mathrm{p}=0,067$ \\
\hline $\begin{array}{c}5-6 \text { Gläser/Woche } \\
\text { vs. gar nicht }\end{array}$ & 1,519 & $\mathrm{p}<0,05$ & 1,203 & $\varnothing$ sign. & 1,272 & $\varnothing$ sign. \\
\hline $\begin{array}{c}\text { 2-4 Gläser/Woche } \\
\text { vs. gar nicht }\end{array}$ & 1,663 & $\mathrm{p}<0,001$ & 1,408 & $\mathrm{p}<0,05$. & 1,545 & $\mathrm{p}<0,01$ \\
\hline $\begin{array}{c}\text { 1 Glas/Woche } \\
\text { vs. gar nicht }\end{array}$ & 1,222 & $\varnothing$ sign. & 1,062 & $\varnothing$ sign. & 1,049 & $\varnothing$ sign. \\
\hline $\begin{array}{c}\text { 1-3 Gläser/Monat } \\
\text { vs. gar nicht }\end{array}$ & 1,336 & $\mathrm{p}<0,05$ & 1,314 & $\mathrm{p}<0,05$. & 1,335 & $\mathrm{p}<0,05$. \\
\hline $\begin{array}{c}<1 \text { Glas/Monat } \\
\text { vs. gar nicht }\end{array}$ & 0,726 & $\mathrm{p}<0,05$ & 0,708 & $\mathrm{p}<0,05$ & 0,759 & $\mathrm{p}=0,053$ \\
\hline $\begin{array}{c}\text { Anpassungsgüte } \\
\text {-2LL }\end{array}$ & & 4470,125 & 4334,015 \\
\hline
\end{tabular}

Tab. IV Kontrollvariablen und Confounder mit OR \& Signifikanz im univariaten und multivariaten Modell am Bsp. des übergreifenden Messwerts der Lebensqualität (Eigenangaben)

\begin{tabular}{|c|c|c|c|c|c|c|}
\hline \multirow{2}{*}{ KINDL-R } & \multicolumn{2}{|c|}{ univariat } & \multicolumn{2}{|c|}{$\begin{array}{l}\text { multivariat: } \\
\text { Modell } 1 \\
\end{array}$} & \multicolumn{2}{|c|}{$\begin{array}{l}\text { multivariat: } \\
\text { Modell 1b }\end{array}$} \\
\hline & $O R$ & Signifikanz & $O R$ & Signifikanz & $O R$ & Signifikanz \\
\hline $\begin{array}{l}\text { Total-KINDL-R } \\
\text { (Eigenangaben) }\end{array}$ & 1,008 & $p<0,05$ & 1,014 & $p \leq 0,001$ & 1,016 & $p<0,001$ \\
\hline Alter & 1,087 & $p<0,001$ & 1,015 & $\varnothing$ sign. & 0,998 & $\varnothing$ sign. \\
\hline Geschlecht & 1,580 & $p<0,001$ & 1,612 & $p<0,001$ & 1,597 & $p<0,001$ \\
\hline BMI & 1,153 & $p<0,001$ & 1,164 & $p<0,001$ & 1,164 & $p<0,001$ \\
\hline \multicolumn{7}{|l|}{ Sozialer Status } \\
\hline mittel vs. hoch & 1,019 & $\varnothing$ sign. & 0,921 & $\varnothing$ sign. & 0,893 & $\varnothing$ sign. \\
\hline niedrig vs. hoch & 1,059 & $\varnothing$ sign. & 0,776 & $p<0,05$ & 0,787 & $p<0,05$ \\
\hline Einschränkungen & 1,500 & $p<0,01$ & 1,108 & $\varnothing$ sign. & 1,123 & $\varnothing$ sign. \\
\hline \multicolumn{7}{|l|}{ Schuldurchlauf } \\
\hline $\begin{array}{l}\text { übersprungen vs. } \\
\text { regulär }\end{array}$ & 0,333 & $p=0,067$ & 0,397 & $\varnothing$ sign. & 0,437 & $\varnothing$ sign. \\
\hline $\begin{array}{l}\text { wiederholt vs. regu- } \\
\text { lär }\end{array}$ & 0,773 & $p<0,05$ & 0,604 & $p<0,001$ & 0,552 & $p \leq 0,001$ \\
\hline
\end{tabular}




\begin{tabular}{|c|c|c|c|c|c|c|}
\hline $\begin{array}{c}\text { Körperliche Leis- } \\
\text { tungsfähigkeit }\end{array}$ & & & & & & \\
\hline gut vs. sehr gut & 1,258 & $p<0,05$ & 1,131 & $\varnothing$ sign. & 1,133 & $\varnothing$ sign. \\
\hline mittel vs. sehr gut & 1,461 & $p \leq 0,001$ & 1,087 & $\varnothing$ sign. & 1,189 & $\varnothing$ sign. \\
\hline $\begin{array}{l}\text { nicht besonders gut } \\
\text { vs. sehr gut }\end{array}$ & 1,712 & $p \leq 0,001$ & 0,994 & $\varnothing$ sign. & 1,118 & $\varnothing$ sign. \\
\hline $\begin{array}{l}\text { gar nicht gut vs. } \\
\text { sehr gut }\end{array}$ & 1,189 & $\varnothing$ sign. & 0,333 & $p=0,057$ & 0,380 & $p=0,099$ \\
\hline \multicolumn{7}{|l|}{ TV/Video } \\
\hline $0,5 \mathrm{~h}$ vs. gar nicht & 1,345 & $\varnothing$ sign. & 1,596 & $p=0,089$ & 1,539 & $\varnothing$ sign. \\
\hline $1-2 \mathrm{~h}$ vs. gar nicht & 1,858 & $p<0,01$ & 1,998 & $p<0,01$ & 1,971 & $p<0,05$ \\
\hline $3-4 h$ vs. gar nicht & 2,007 & $p<0,01$ & 2,095 & $p<0,01$ & 2,195 & $p<0,01$ \\
\hline$>4 \mathrm{~h}$ vs. gar nicht & 1,801 & $p<0,05$ & 1,506 & $\varnothing$ sign. & 1,560 & $\varnothing$ sign. \\
\hline \multicolumn{7}{|l|}{ Computer } \\
\hline $0,5 \mathrm{~h}$ vs. gar nicht & 0,928 & $\varnothing$ sign. & 0,900 & $\varnothing$ sign. & 0,890 & $\varnothing$ sign. \\
\hline $1-2 \mathrm{~h}$ vs. gar nicht & 1,010 & $\varnothing$ sign. & 0,851 & $\varnothing$ sign. & 0,824 & $p=0,097$ \\
\hline $3-4 h$ vs. gar nicht & 1,211 & $\varnothing$ sign. & 0,859 & $\varnothing$ sign. & 0,856 & $\varnothing$ sign. \\
\hline$>4 \mathrm{~h}$ vs. gar nicht & 1,889 & $p<0,001$ & 1,274 & $\varnothing$ sign. & 1,412 & $p=0,074$ \\
\hline \multicolumn{7}{|l|}{ Bier } \\
\hline $\begin{array}{l}>=1 \mathrm{Glas} / \mathrm{d} \\
\text { vs. gar nicht }\end{array}$ & 2,486 & $p<0,01$ & 1,800 & $p=0,070$ & 1,891 & $p=0,074$ \\
\hline $\begin{array}{l}\text { 5-6 Gläser/Woche } \\
\text { vs. gar nicht }\end{array}$ & 1,519 & $p<0,05$ & 1,203 & $\varnothing$ sign. & 1,241 & $\varnothing$ sign. \\
\hline $\begin{array}{l}\text { 2-4 Gläser/Woche } \\
\text { vs. gar nicht }\end{array}$ & 1,663 & $p<0,001$ & 1,408 & $p<0,05$ & 1,516 & $p<0,01$ \\
\hline $\begin{array}{l}1 \text { Glas/Woche } \\
\text { vs. gar nicht }\end{array}$ & 1,222 & $\varnothing$ sign. & 1,062 & $\varnothing$ sign. & 1,000 & $\varnothing$ sign. \\
\hline $\begin{array}{l}\text { 1-3 Gläser/Monat } \\
\text { vs. gar nicht }\end{array}$ & 1,336 & $p<0,05$ & 1,314 & $p<0,05$ & 1,330 & $p<0,05$ \\
\hline $\begin{array}{l}<1 \text { Glas/Monat } \\
\text { vs. gar nicht }\end{array}$ & 0,726 & $p<0,05$ & 0,708 & $p<0,05$ & 0,764 & $p=0,060$ \\
\hline $\begin{array}{c}\text { Anpassungsgüte } \\
-2 \mathrm{LL}\end{array}$ & & & \multicolumn{2}{|c|}{4470,125} & \multicolumn{2}{|c|}{4331,836} \\
\hline
\end{tabular}


Tab. V Kontrollvariablen und Confounder mit OR \& Signifikanz im univariaten und multivariaten Modell am Bsp. Hyperaktivität (Eigenangaben)

\begin{tabular}{|c|c|c|c|c|c|c|}
\hline \multirow{2}{*}{$S D Q$} & \multicolumn{2}{|c|}{ univariat } & \multicolumn{2}{|c|}{$\begin{array}{c}\text { multivariat: } \\
\text { Modell } 1\end{array}$} & \multicolumn{2}{|c|}{$\begin{array}{l}\text { multivariat: } \\
\text { Modell 1b }\end{array}$} \\
\hline & $O R$ & Signifikanz & OR & Signifikanz & OR & Signifikanz \\
\hline $\begin{array}{l}\text { Hyperaktivität } \\
\text { (Eigenangaben) }\end{array}$ & 0,944 & $p<0,01$ & 0,934 & $p \leq 0,001$ & 0,926 & $p<0,001$ \\
\hline Alter & 1,087 & $p<0,001$ & 1,002 & $\varnothing$ sign. & 0,978 & $\varnothing$ sign. \\
\hline Geschlecht & 1,580 & $p<0,001$ & 1,678 & $p<0,001$ & 1,651 & $p<0,001$ \\
\hline$B M I$ & 1,153 & $p<0,001$ & 1,163 & $p<0,001$ & 1,167 & $p<0,001$ \\
\hline \multicolumn{7}{|l|}{ Sozialer Status } \\
\hline mittel vs. hoch & 1,019 & $\varnothing$ sign. & 0,915 & $\varnothing$ sign. & 0,874 & $\varnothing$ sign. \\
\hline niedrig vs. hoch & 1,059 & $\varnothing$ sign. & 0,770 & $p<0,05$ & 0,776 & $p<0,05$ \\
\hline Einschränkungen & 1,500 & $p<0,01$ & 1,108 & $\varnothing$ sign. & 1,036 & $\varnothing$ sign. \\
\hline \multicolumn{7}{|l|}{ Schuldurchlauf } \\
\hline $\begin{array}{c}\text { übersprungen vs. } \\
\text { regulär }\end{array}$ & 0,333 & $p=0,067$ & 0,397 & $\varnothing$ sign. & 0,445 & $\varnothing$ sign. \\
\hline $\begin{array}{l}\text { wiederholt vs. regu- } \\
\text { lär }\end{array}$ & 0,773 & $p<0,05$ & 0,604 & $p<0,001$ & 0,590 & $p \leq 0,001$ \\
\hline \multicolumn{7}{|l|}{$\begin{array}{c}\text { Körperliche Leis- } \\
\text { tungsfähigkeit }\end{array}$} \\
\hline gut vs. sehr gut & 1,258 & $p<0,05$ & 1,131 & $\varnothing$ sign. & 1,085 & $\varnothing$ sign. \\
\hline mittel vs. sehr gut & 1,461 & $p \leq 0,001$ & 1,087 & $\varnothing$ sign. & 1,096 & $\varnothing$ sign. \\
\hline $\begin{array}{l}\text { nicht besonders gut } \\
\text { vs. sehr gut }\end{array}$ & 1,712 & $p \leq 0,001$ & 0,994 & $\varnothing$ sign. & 1,003 & $\varnothing$ sign. \\
\hline $\begin{array}{l}\text { gar nicht gut vs. } \\
\text { sehr gut }\end{array}$ & 1,189 & $\varnothing$ sign. & 0,333 & $p=0,057$ & 0,348 & $p=0,073$ \\
\hline \multicolumn{7}{|l|}{ TV/Video } \\
\hline $0,5 \mathrm{~h}$ vs. gar nicht & 1,345 & $\varnothing$ sign. & 1,596 & $p=0,089$ & 1,603 & $p=0,089$ \\
\hline $1-2 \mathrm{~h}$ vs. gar nicht & 1,858 & $p<0,01$ & 1,998 & $p<0,01$ & 2,014 & $p<0,01$ \\
\hline $3-4$ h vs. gar nicht & 2,007 & $p<0,01$ & 2,095 & $p<0,01$ & 2,193 & $p<0,01$ \\
\hline$>4 \mathrm{~h}$ vs. gar nicht & 1,801 & $p<0,05$ & 1,506 & $\varnothing$ sign. & 1,559 & $\varnothing$ sign. \\
\hline \multicolumn{7}{|l|}{ Computer } \\
\hline $0,5 \mathrm{~h}$ vs. gar nicht & 0,928 & $\varnothing$ sign. & 0,900 & $\varnothing$ sign. & 0,859 & $\varnothing$ sign. \\
\hline $1-2 \mathrm{~h}$ vs. gar nicht & 1,010 & $\varnothing$ sign. & 0,851 & $\varnothing$ sign. & 0,800 & $p=0,053$ \\
\hline 3-4h vs. gar nicht & 1,211 & $\varnothing$ sign. & 0,859 & $\varnothing$ sign. & 0,806 & $\varnothing$ sign. \\
\hline$>4 \mathrm{~h}$ vs. gar nicht & 1,889 & $p<0,001$ & 1,274 & $\varnothing$ sign. & 1,416 & $p=0,069$ \\
\hline
\end{tabular}




\begin{tabular}{|c|c|c|c|c|c|c|}
\hline Bier & \multicolumn{7}{|c|}{$\begin{array}{c}\text { >= 1 Glas/d } \\
\text { vs. gar nicht }\end{array}$} & 2,486 & $\mathrm{p}<0,01$ & 1,800 & $\mathrm{p}=0,070$ & 1,861 & $\mathrm{p}=0,079$ \\
\hline $\begin{array}{c}5-6 \text { Gläser/Woche } \\
\text { vs. gar nicht }\end{array}$ & 1,519 & $\mathrm{p}<0,05$ & 1,203 & $\varnothing$ sign. & 1,238 & $\varnothing$ sign. \\
\hline $\begin{array}{c}\text { 2-4 Gläser/Woche } \\
\text { vs. gar nicht }\end{array}$ & 1,663 & $\mathrm{p}<0,001$ & 1,408 & $\mathrm{p}<0,05$. & 1,534 & $\mathrm{p}<0,01$ \\
\hline $\begin{array}{c}\text { 1 Glas/Woche } \\
\text { vs. gar nicht }\end{array}$ & 1,222 & $\varnothing$ sign. & 1,062 & $\varnothing$ sign. & 1,082 & $\varnothing$ sign. \\
\hline $\begin{array}{c}\text { 1-3 Gläser/Monat } \\
\text { vs. gar nicht }\end{array}$ & 1,336 & $\mathrm{p}<0,05$ & 1,314 & $\mathrm{p}<0,05$. & 1,334 & $\mathrm{p}<0,05$. \\
\hline $\begin{array}{c}<1 \text { Glas/Monat } \\
\text { vs. gar nicht }\end{array}$ & 0,726 & $\mathrm{p}<0,05$ & 0,708 & $\mathrm{p}<0,05$ & 0,763 & $\mathrm{p}=0,056$ \\
\hline $\begin{array}{c}\text { Anpassungsgüte } \\
\text {-2LL }\end{array}$ & & 4470,125 & 4373,631 \\
\hline
\end{tabular}

Tab. VI Kontrollvariablen und Confounder mit OR \& Signifikanz im univariaten und multivariaten Modell am Bsp. körperliches Wohlbefinden (Elternangaben)

\begin{tabular}{|c|c|c|c|c|c|c|}
\hline \multirow{2}{*}{ KINDL-R } & \multicolumn{2}{|c|}{ univariat } & \multicolumn{2}{|c|}{$\begin{array}{c}\text { multivariat: } \\
\text { Modell } 1\end{array}$} & \multicolumn{2}{|c|}{$\begin{array}{l}\text { multivariat: } \\
\text { Modell 1b }\end{array}$} \\
\hline & $O R$ & Signifikanz & OR & Signifikanz & OR & Signifikanz \\
\hline $\begin{array}{c}\text { Körperliches WB } \\
\text { (Elternangaben) }\end{array}$ & 1,005 & $p<0,05$ & 1,007 & $p<0,01$ & 1,006 & $p<0,05$ \\
\hline Alter & 1,087 & $p<0,001$ & 1,000 & $\varnothing$ sign. & 0,988 & $\varnothing$ sign. \\
\hline Geschlecht & 1,580 & $p<0,001$ & 1,588 & $p<0,001$ & 1,564 & $p<0,001$ \\
\hline$B M I$ & 1,153 & $p<0,001$ & 1,167 & $p<0,001$ & 1,168 & $p<0,001$ \\
\hline \multicolumn{7}{|l|}{ Sozialer Status } \\
\hline mittel vs. hoch & 1,019 & $\varnothing$ sign. & 0,924 & $\varnothing$ sign. & 0,878 & $\varnothing$ sign. \\
\hline niedrig vs. hoch & 1,059 & $\varnothing$ sign. & 0,783 & $p<0,05$ & 0,781 & $p<0,05$ \\
\hline Einschränkungen & 1,500 & $p<0,01$ & 1,108 & $\varnothing$ sign. & 1,103 & $\varnothing$ sign. \\
\hline \multicolumn{7}{|l|}{ Schuldurchlauf } \\
\hline $\begin{array}{c}\text { übersprungen vs. } \\
\text { regulär }\end{array}$ & 0,333 & $p=0,067$ & 0,397 & $\varnothing$ sign. & 0,459 & $\varnothing$ sign. \\
\hline $\begin{array}{c}\text { wiederholt vs. regu- } \\
\text { lär }\end{array}$ & 0,773 & $p<0,05$ & 0,604 & $p<0,001$ & 0,580 & $p<0,001$ \\
\hline
\end{tabular}




\begin{tabular}{|c|c|c|c|c|c|c|}
\hline $\begin{array}{c}\text { Körperliche Leis- } \\
\text { tungsfähigkeit }\end{array}$ & & & & & & \\
\hline gut vs. sehr gut & 1,258 & $p<0,05$ & 1,131 & $\varnothing$ sign. & 1,074 & $\varnothing$ sign. \\
\hline mittel vs. sehr gut & 1,461 & $p \leq 0,001$ & 1,087 & $\varnothing$ sign. & 1,101 & $\varnothing$ sign. \\
\hline $\begin{array}{l}\text { nicht besonders gut } \\
\text { vs. sehr gut }\end{array}$ & 1,712 & $p \leq 0,001$ & 0,994 & $\varnothing$ sign. & 0,969 & $\varnothing$ sign. \\
\hline $\begin{array}{l}\text { gar nicht gut vs. } \\
\text { sehr gut }\end{array}$ & 1,189 & $\varnothing$ sign. & 0,333 & $p=0,057$ & 0,330 & $p=0,060$ \\
\hline \multicolumn{7}{|l|}{ TV/Video } \\
\hline $0,5 \mathrm{~h}$ vs. gar nicht & 1,345 & $\varnothing$ sign. & 1,596 & $p=0,089$ & 1,552 & $\varnothing$ sign. \\
\hline $1-2 \mathrm{~h}$ vs. gar nicht & 1,858 & $p<0,01$ & 1,998 & $p<0,01$ & 1,964 & $p<0,05$ \\
\hline 3-4h vs. gar nicht & 2,007 & $p<0,01$ & 2,095 & $p<0,01$ & 2,142 & $p<0,01$ \\
\hline$>4 \mathrm{~h}$ vs. gar nicht & 1,801 & $p<0,05$ & 1,506 & $\varnothing$ sign. & 1,522 & $\varnothing$ sign. \\
\hline \multicolumn{7}{|l|}{ Computer } \\
\hline $0,5 \mathrm{~h}$ vs. gar nicht & 0,928 & $\varnothing$ sign. & 0,900 & $\varnothing$ sign. & 0,893 & $\varnothing$ sign. \\
\hline 1-2h vs. gar nicht & 1,010 & $\varnothing$ sign. & 0,851 & $\varnothing$ sign. & 0,818 & $p=0,086$ \\
\hline 3-4h vs. gar nicht & 1,211 & $\varnothing$ sign. & 0,859 & $\varnothing$ sign. & 0,848 & $\varnothing$ sign. \\
\hline$>4 \mathrm{~h}$ vs. gar nicht & 1,889 & $p<0,001$ & 1,274 & $\varnothing$ sign. & 1,442 & $p=0,057$ \\
\hline \multicolumn{7}{|l|}{ Bier } \\
\hline $\begin{array}{l}>=1 \mathrm{Glas} / \mathrm{d} \\
\text { vs. gar nicht }\end{array}$ & 2,486 & $p<0,01$ & 1,800 & $p=0,070$ & 1,816 & $p=0,093$ \\
\hline $\begin{array}{l}\text { 5-6 Gläser/Woche } \\
\text { vs. gar nicht }\end{array}$ & 1,519 & $p<0,05$ & 1,203 & $\varnothing$ sign. & 1,219 & $\varnothing$ sign. \\
\hline $\begin{array}{l}\text { 2-4 Gläser/Woche } \\
\text { vs. gar nicht }\end{array}$ & 1,663 & $p<0,001$ & 1,408 & $p<0,05$ & 1,443 & $p<0,05$ \\
\hline $\begin{array}{l}1 \text { Glas/Woche } \\
\text { vs. gar nicht }\end{array}$ & 1,222 & $\varnothing$ sign. & 1,062 & $\varnothing$ sign. & 1,074 & $\varnothing$ sign. \\
\hline $\begin{array}{l}\text { 1-3 Gläser/Monat } \\
\text { vs. gar nicht }\end{array}$ & 1,336 & $p<0,05$ & 1,314 & $p<0,05$ & 1,237 & $\varnothing$ sign. \\
\hline $\begin{array}{l}<1 \text { Glas/Monat } \\
\text { vs. gar nicht }\end{array}$ & 0,726 & $p<0,05$ & 0,708 & $p<0,05$ & 0,738 & $p<0,05$ \\
\hline $\begin{array}{c}\text { Anpassungsgüte } \\
-2 L L\end{array}$ & & & \multicolumn{2}{|c|}{4470,125} & \multicolumn{2}{|c|}{4316,132} \\
\hline
\end{tabular}


Tab. VII Kontrollvariablen und Confounder mit OR \& Signifikanz im univariaten und multivariaten Modell am Bsp. Selbstwert (Elternangaben)

\begin{tabular}{|c|c|c|c|c|c|c|}
\hline \multirow{2}{*}{ KINDL-R } & \multicolumn{2}{|c|}{ univariat } & \multicolumn{2}{|c|}{$\begin{array}{c}\text { multivariat: } \\
\text { Modell } 1\end{array}$} & \multicolumn{2}{|c|}{$\begin{array}{l}\text { multivariat: } \\
\text { Modell 1b }\end{array}$} \\
\hline & OR & Signifikanz & OR & Signifikanz & $O R$ & Signifikanz \\
\hline $\begin{array}{c}\text { Selbstwert } \\
\text { (Elternangaben) }\end{array}$ & 1,009 & $p \leq 0,001$ & 1,011 & $p<0,001$ & 1,012 & $p<0,001$ \\
\hline Alter & 1,087 & $p<0,001$ & 0,996 & $\varnothing$ sign. & 0,978 & $\varnothing$ sign. \\
\hline Geschlecht & 1,580 & $p<0,001$ & 1,665 & $p<0,001$ & 1,620 & $p<0,001$ \\
\hline$B M I$ & 1,153 & $p<0,001$ & 1,163 & $p<0,001$ & 1,165 & $p<0,001$ \\
\hline \multicolumn{7}{|l|}{ Sozialer Status } \\
\hline mittel vs. hoch & 1,019 & $\varnothing$ sign. & 0,911 & $\varnothing$ sign. & 0,862 & $\varnothing$ sign. \\
\hline niedrig vs. hoch & 1,059 & $\varnothing$ sign. & 0,768 & $p<0,05$ & 0,764 & $p<0,05$ \\
\hline Einschränkungen & 1,500 & $p<0,01$ & 1,108 & $\varnothing$ sign. & 1,167 & $\varnothing$ sign. \\
\hline \multicolumn{7}{|l|}{ Schuldurchlauf } \\
\hline $\begin{array}{c}\text { übersprungen vs. } \\
\text { regulär }\end{array}$ & 0,333 & $p=0,067$ & 0,397 & $\varnothing$ sign. & 0,460 & $\varnothing$ sign. \\
\hline $\begin{array}{l}\text { wiederholt vs. regu- } \\
\text { lär }\end{array}$ & 0,773 & $p<0,05$ & 0,604 & $p<0,001$ & 0,593 & $p<0,001$ \\
\hline \multicolumn{7}{|l|}{$\begin{array}{l}\text { Körperliche Leis- } \\
\text { tungsfähigkeit }\end{array}$} \\
\hline gut vs. sehr gut & 1,258 & $p<0,05$ & 1,131 & $\varnothing$ sign. & 1,071 & $\varnothing$ sign. \\
\hline mittel vs. sehr gut & 1,461 & $p \leq 0,001$ & 1,087 & $\varnothing$ sign. & 1,141 & $\varnothing$ sign. \\
\hline $\begin{array}{l}\text { nicht besonders gut } \\
\text { vs. sehr gut }\end{array}$ & 1,712 & $p \leq 0,001$ & 0,994 & $\varnothing$ sign. & 0,994 & $\varnothing$ sign. \\
\hline $\begin{array}{c}\text { gar nicht gut vs. } \\
\text { sehr gut }\end{array}$ & 1,189 & $\varnothing$ sign. & 0,333 & $p=0,057$ & 0,368 & $p=0,088$ \\
\hline \multicolumn{7}{|l|}{ TV/Video } \\
\hline $0,5 \mathrm{~h}$ vs. gar nicht & 1,345 & $\varnothing$ sign. & 1,596 & $p=0,089$ & 1,514 & $\varnothing$ sign. \\
\hline $1-2 \mathrm{~h}$ vs. gar nicht & 1,858 & $p<0,01$ & 1,998 & $p<0,01$ & 1,901 & $p<0,05$ \\
\hline 3-4h vs. gar nicht & 2,007 & $p<0,01$ & 2,095 & $p<0,01$ & 2,133 & $p<0,01$ \\
\hline$>4 \mathrm{~h}$ vs. gar nicht & 1,801 & $p<0,05$ & 1,506 & $\varnothing$ sign. & 1,407 & $\varnothing$ sign. \\
\hline \multicolumn{7}{|l|}{ Computer } \\
\hline $0,5 \mathrm{~h}$ vs. gar nicht & 0,928 & $\varnothing$ sign. & 0,900 & $\varnothing$ sign. & 0,883 & $\varnothing$ sign. \\
\hline $1-2 \mathrm{~h}$ vs. gar nicht & 1,010 & $\varnothing$ sign. & 0,851 & $\varnothing$ sign. & 0,812 & $p=0,075$ \\
\hline $3-4$ h vs. gar nicht & 1,211 & $\varnothing$ sign. & 0,859 & $\varnothing$ sign. & 0,842 & $\varnothing$ sign. \\
\hline$>4 \mathrm{~h}$ vs. gar nicht & 1,889 & $p<0,001$ & 1,274 & $\varnothing$ sign. & 1,437 & $p<0,05$ \\
\hline
\end{tabular}




\begin{tabular}{|c|c|c|c|c|c|c|}
\hline Bier & & & & & & \\
\hline $\begin{array}{l}>=1 \mathrm{Glas} / \mathrm{d} \\
\text { vs. gar nicht }\end{array}$ & 2,486 & $p<0,01$ & 1,800 & $p=0,070$ & 1,894 & $p=0,075$ \\
\hline $\begin{array}{l}\text { 5-6 Gläser/Woche } \\
\text { vs. gar nicht }\end{array}$ & 1,519 & $p<0,05$ & 1,203 & $\varnothing$ sign. & 1,262 & $\varnothing$ sign. \\
\hline $\begin{array}{l}\text { 2-4 Gläser/Woche } \\
\text { vs. gar nicht }\end{array}$ & 1,663 & $p<0,001$ & 1,408 & $p<0,05$ & 1,557 & $p<0,01$ \\
\hline $\begin{array}{l}1 \text { Glas/Woche } \\
\text { vs. gar nicht }\end{array}$ & 1,222 & $\varnothing$ sign. & 1,062 & $\varnothing$ sign. & 1,088 & $\varnothing$ sign. \\
\hline $\begin{array}{l}\text { 1-3 Gläser/Monat } \\
\text { vs. gar nicht }\end{array}$ & 1,336 & $p<0,05$ & 1,314 & $p<0,05$ & 1,329 & $p<0,05$ \\
\hline $\begin{array}{l}<1 \text { Glas/Monat } \\
\text { vs. gar nicht }\end{array}$ & 0,726 & $p<0,05$ & 0,708 & $p<0,05$ & 0,770 & $p=0,068$ \\
\hline $\begin{array}{c}\text { Anpassungsgüte } \\
-2 \mathrm{LL}\end{array}$ & & & \multicolumn{2}{|c|}{4470,125} & \multicolumn{2}{|c|}{4303,248} \\
\hline
\end{tabular}

Tab. VIII Kontrollvariablen und Confounder mit OR \& Signifikanz im univariaten und multivariaten Modell am Bsp. Familie (Elternangaben)

\begin{tabular}{|c|c|c|c|c|c|c|}
\hline \multirow{2}{*}{ KINDL-R } & \multicolumn{2}{|c|}{ univariat } & \multicolumn{2}{|c|}{$\begin{array}{l}\text { multivariat: } \\
\text { Modell } 1\end{array}$} & \multicolumn{2}{|c|}{$\begin{array}{l}\text { multivariat: } \\
\text { Modell 1b }\end{array}$} \\
\hline & $O R$ & Signifikanz & $O R$ & Signifikanz & $O R$ & Signifikanz \\
\hline $\begin{array}{c}\text { Familie } \\
\text { (Elternangaben) }\end{array}$ & 1,008 & $p<0,01$ & 1,010 & $p<0,001$ & 1,012 & $p<0,001$ \\
\hline Alter & 1,087 & $p<0,001$ & 0,997 & $\varnothing$ sign. & 0,977 & $\varnothing$ sign. \\
\hline Geschlecht & 1,580 & $p<0,001$ & 1,662 & $p<0,001$ & 1,588 & $p<0,001$ \\
\hline$B M I$ & 1,153 & $p<0,001$ & 1,162 & $p<0,001$ & 1,166 & $p<0,001$ \\
\hline \multicolumn{7}{|l|}{ Sozialer Status } \\
\hline mittel vs. hoch & 1,019 & $\varnothing$ sign. & 0,901 & $\varnothing$ sign. & 0,859 & $\varnothing$ sign. \\
\hline niedrig vs. hoch & 1,059 & $\varnothing$ sign. & 0,761 & $p<0,05$ & 0,765 & $p<0,05$ \\
\hline Einschränkungen & 1,500 & $p<0,01$ & 1,108 & $\varnothing$ sign. & 1,146 & $\varnothing$ sign. \\
\hline \multicolumn{7}{|l|}{ Schuldurchlauf } \\
\hline $\begin{array}{l}\text { übersprungen vs. } \\
\text { regulär }\end{array}$ & 0,333 & $p=0,067$ & 0,397 & $\varnothing$ sign. & 0,470 & $\varnothing$ sign. \\
\hline $\begin{array}{c}\text { wiederholt vs. regu- } \\
\text { lär }\end{array}$ & 0,773 & $p<0,05$ & 0,604 & $p<0,001$ & 0,592 & $p<0,001$ \\
\hline
\end{tabular}




\begin{tabular}{|c|c|c|c|c|c|c|}
\hline $\begin{array}{c}\text { Körperliche Leis- } \\
\text { tungsfähigkeit }\end{array}$ & & & & & & \\
\hline gut vs. sehr gut & 1,258 & $p<0,05$ & 1,131 & $\varnothing$ sign. & 1,052 & $\varnothing$ sign. \\
\hline mittel vs. sehr gut & 1,461 & $p \leq 0,001$ & 1,087 & $\varnothing$ sign. & 1,093 & $\varnothing$ sign. \\
\hline $\begin{array}{l}\text { nicht besonders gut } \\
\text { vs. sehr gut }\end{array}$ & 1,712 & $p \leq 0,001$ & 0,994 & $\varnothing$ sign. & 0,917 & $\varnothing$ sign. \\
\hline $\begin{array}{l}\text { gar nicht gut vs. } \\
\text { sehr gut }\end{array}$ & 1,189 & $\varnothing$ sign. & 0,333 & $p=0,057$ & 0,331 & $p=0,061$ \\
\hline \multicolumn{7}{|l|}{ TV/Video } \\
\hline $0,5 \mathrm{~h}$ vs. gar nicht & 1,345 & $\varnothing$ sign. & 1,596 & $p=0,089$ & 1,545 & $\varnothing$ sign. \\
\hline $1-2 \mathrm{~h}$ vs. gar nicht & 1,858 & $p<0,01$ & 1,998 & $p<0,01$ & 1,914 & $p<0,05$ \\
\hline $3-4 h$ vs. gar nicht & 2,007 & $p<0,01$ & 2,095 & $p<0,01$ & 2,079 & $p<0,01$ \\
\hline$>4 \mathrm{~h}$ vs. gar nicht & 1,801 & $p<0,05$ & 1,506 & $\varnothing$ sign. & 1,358 & $\varnothing$ sign. \\
\hline \multicolumn{7}{|l|}{ Computer } \\
\hline $0,5 \mathrm{~h}$ vs. gar nicht & 0,928 & $\varnothing$ sign. & 0,900 & $\varnothing$ sign. & 0,910 & $\varnothing$ sign. \\
\hline $1-2 \mathrm{~h}$ vs. gar nicht & 1,010 & $\varnothing$ sign. & 0,851 & $\varnothing$ sign. & 0,833 & $\varnothing$ sign. \\
\hline $3-4 h$ vs. gar nicht & 1,211 & $\varnothing$ sign. & 0,859 & $\varnothing$ sign. & 0,868 & $\varnothing$ sign. \\
\hline$>4 \mathrm{~h}$ vs. gar nicht & 1,889 & $p<0,001$ & 1,274 & $\varnothing$ sign. & 1,504 & $p<0,05$ \\
\hline \multicolumn{7}{|l|}{ Bier } \\
\hline $\begin{array}{l}>=1 \mathrm{Glas} / \mathrm{d} \\
\text { vs. gar nicht }\end{array}$ & 2,486 & $p<0,01$ & 1,800 & $p=0,070$ & 2,002 & $p=0,052$ \\
\hline $\begin{array}{l}\text { 5-6 Gläser/Woche } \\
\text { vs. gar nicht }\end{array}$ & 1,519 & $p<0,05$ & 1,203 & $\varnothing$ sign. & 1,312 & $\varnothing$ sign. \\
\hline $\begin{array}{l}\text { 2-4 Gläser/Woche } \\
\text { vs. gar nicht }\end{array}$ & 1,663 & $p<0,001$ & 1,408 & $\mathrm{p}<0,05$ & 1,568 & $p<0,01$ \\
\hline $\begin{array}{l}1 \text { Glas/Woche } \\
\text { vs. gar nicht }\end{array}$ & 1,222 & $\varnothing$ sign. & 1,062 & $\varnothing$ sign. & 1,090 & $\varnothing$ sign. \\
\hline $\begin{array}{l}\text { 1-3 Gläser/Monat } \\
\text { vs. gar nicht }\end{array}$ & 1,336 & $p<0,05$ & 1,314 & $p<0,05$ & 1,295 & $p=0,066$ \\
\hline $\begin{array}{l}<1 \text { Glas/Monat } \\
\text { vs. gar nicht }\end{array}$ & 0,726 & $p<0,05$ & 0,708 & $p<0,05$ & 0,777 & $p=0,075$ \\
\hline $\begin{array}{c}\text { Anpassungsgüte } \\
-2 \mathrm{LL}\end{array}$ & & & \multicolumn{2}{|c|}{4470,125} & \multicolumn{2}{|c|}{4332,536} \\
\hline
\end{tabular}


Tab. IX Kontrollvariablen und Confounder mit OR \& Signifikanz im univariaten und multivariaten Modell am Bsp. des übergreifenden Messwerts der Lebensqualität (Elternangaben)

\begin{tabular}{|c|c|c|c|c|c|c|}
\hline \multirow{2}{*}{ KINDL-R } & \multicolumn{2}{|c|}{ univariat } & \multicolumn{2}{|c|}{$\begin{array}{l}\text { multivariat: } \\
\text { Modell } 1\end{array}$} & \multicolumn{2}{|c|}{$\begin{array}{l}\text { multivariat: } \\
\text { Modell 1b }\end{array}$} \\
\hline & OR & Signifikanz & OR & Signifikanz & $O R$ & Signifikanz \\
\hline $\begin{array}{l}\text { Total-KINDL-R } \\
\text { (Elternangaben) }\end{array}$ & 1,010 & $p<0,01$ & 1,016 & $p<0,001$ & 1,017 & $p<0,001$ \\
\hline Alter & 1,087 & $p<0,001$ & 1,000 & $\varnothing$ sign. & 0,986 & $\varnothing$ sign. \\
\hline Geschlecht & 1,580 & $p<0,001$ & 1,619 & $p<0,001$ & 1,580 & $p<0,001$ \\
\hline BMI & 1,153 & $p<0,001$ & 1,165 & $p<0,001$ & 1,166 & $p<0,001$ \\
\hline \multicolumn{7}{|l|}{ Sozialer Status } \\
\hline mittel vs. hoch & 1,019 & $\varnothing$ sign. & 0,910 & $\varnothing$ sign. & 0,865 & $\varnothing$ sign. \\
\hline niedrig vs. hoch & 1,059 & $\varnothing$ sign. & 0,789 & $p<0,05$ & 0,785 & $p<0,05$ \\
\hline Einschränkungen & 1,500 & $p<0,01$ & 1,108 & $\varnothing$ sign. & 1,193 & $\varnothing$ sign. \\
\hline \multicolumn{7}{|l|}{ Schuldurchlauf } \\
\hline $\begin{array}{l}\text { übersprungen vs. } \\
\text { regulär }\end{array}$ & 0,333 & $p=0,067$ & 0,397 & $\varnothing$ sign. & 0,456 & $\varnothing$ sign. \\
\hline $\begin{array}{c}\text { wiederholt vs. regu- } \\
\text { lär }\end{array}$ & 0,773 & $p<0,05$ & 0,604 & $p<0,001$ & 0,595 & $p<0,001$ \\
\hline \multicolumn{7}{|l|}{$\begin{array}{c}\text { Körperliche Leis- } \\
\text { tungsfähigkeit }\end{array}$} \\
\hline gut vs. sehr gut & 1,258 & $p<0,05$ & 1,131 & $\varnothing$ sign. & 1,072 & $\varnothing$ sign. \\
\hline mittel vs. sehr gut & 1,461 & $p \leq 0,001$ & 1,087 & $\varnothing$ sign. & 1,141 & $\varnothing$ sign. \\
\hline $\begin{array}{l}\text { nicht besonders gut } \\
\text { vs. sehr gut }\end{array}$ & 1,712 & $p \leq 0,001$ & 0,994 & $\varnothing$ sign. & 1,021 & $\varnothing$ sign. \\
\hline $\begin{array}{l}\text { gar nicht gut vs. } \\
\text { sehr gut }\end{array}$ & 1,189 & $\varnothing$ sign. & 0,333 & $p=0,057$ & 0,366 & $p=0,088$ \\
\hline \multicolumn{7}{|l|}{ TV/Video } \\
\hline $0,5 \mathrm{~h}$ vs. gar nicht & 1,345 & $\varnothing$ sign. & 1,596 & $p=0,089$ & 1,514 & $\varnothing$ sign. \\
\hline $1-2 \mathrm{~h}$ vs. gar nicht & 1,858 & $p<0,01$ & 1,998 & $p<0,01$ & 1,907 & $p<0,05$ \\
\hline $3-4 \mathrm{~h}$ vs. gar nicht & 2,007 & $p<0,01$ & 2,095 & $p<0,01$ & 2,086 & $p<0,01$ \\
\hline$>4 \mathrm{~h}$ vs. gar nicht & 1,801 & $p<0,05$ & 1,506 & $\varnothing$ sign. & 1,406 & $\varnothing$ sign. \\
\hline \multicolumn{7}{|l|}{ Computer } \\
\hline $0,5 \mathrm{~h}$ vs. gar nicht & 0,928 & $\varnothing$ sign. & 0,900 & $\varnothing$ sign. & 0,900 & $\varnothing$ sign. \\
\hline $1-2 \mathrm{~h}$ vs. gar nicht & 1,010 & $\varnothing$ sign. & 0,851 & $\varnothing$ sign. & 0,824 & $p=0,097$ \\
\hline $3-4$ h vs. gar nicht & 1,211 & $\varnothing$ sign. & 0,859 & $\varnothing$ sign. & 0,861 & $\varnothing$ sign. \\
\hline$>4 \mathrm{~h}$ vs. gar nicht & 1,889 & $p<0,001$ & 1,274 & $\varnothing$ sign. & 1,502 & $p<0,05$ \\
\hline
\end{tabular}




\begin{tabular}{|c|c|c|c|c|c|c|}
\hline Bier & & & & & & \\
\hline $\begin{array}{l}>=1 \mathrm{Glas} / \mathrm{d} \\
\text { vs. gar nicht }\end{array}$ & 2,486 & $p<0,01$ & 1,800 & $p=0,070$ & 1,888 & $p=0,075$ \\
\hline $\begin{array}{l}\text { 5-6 Gläser/Woche } \\
\text { vs. gar nicht }\end{array}$ & 1,519 & $p<0,05$ & 1,203 & $\varnothing$ sign. & 1,219 & $\varnothing$ sign. \\
\hline $\begin{array}{l}\text { 2-4 Gläser/Woche } \\
\text { vs. gar nicht }\end{array}$ & 1,663 & $p<0,001$ & 1,408 & $p<0,05$ & 1,511 & $p<0,01$ \\
\hline $\begin{array}{l}1 \text { Glas/Woche } \\
\text { vs. gar nicht }\end{array}$ & 1,222 & $\varnothing$ sign. & 1,062 & $\varnothing$ sign. & 1,052 & $\varnothing$ sign. \\
\hline $\begin{array}{l}\text { 1-3 Gläser/Monat } \\
\text { vs. gar nicht }\end{array}$ & 1,336 & $p<0,05$ & 1,314 & $p<0,05$ & 1,256 & $\varnothing$ sign. \\
\hline $\begin{array}{l}<1 \text { Glas/Monat } \\
\text { vs. gar nicht }\end{array}$ & 0,726 & $p<0,05$ & 0,708 & $p<0,05$ & 0,770 & $p=0,065$ \\
\hline $\begin{array}{c}\text { Anpassungsgüte } \\
-2 \mathrm{LL}\end{array}$ & & & \multicolumn{2}{|c|}{4470,125} & \multicolumn{2}{|c|}{4337,149} \\
\hline
\end{tabular}

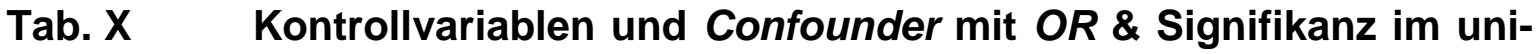
variaten und multivariaten Modell am Bsp. emotionale Probleme (Elternangaben)

\begin{tabular}{|c|c|c|c|c|c|c|}
\hline \multirow{2}{*}{$S D Q$} & \multicolumn{2}{|c|}{ univariat } & \multicolumn{2}{|c|}{$\begin{array}{l}\text { multivariat: } \\
\text { Modell } 1 \\
\end{array}$} & \multicolumn{2}{|c|}{$\begin{array}{l}\text { multivariat: } \\
\text { Modell 1b }\end{array}$} \\
\hline & $O R$ & Signifikanz & OR & Signifikanz & OR & Signifikanz \\
\hline $\begin{array}{c}\text { Emotionale Prob- } \\
\text { leme } \\
\text { (Elternangaben) }\end{array}$ & 0,931 & $p \leq 0,001$ & 0,936 & $p<0,01$ & 0,949 & $p<0,05$ \\
\hline Alter & 1,087 & $p<0,001$ & 0,997 & $\varnothing$ sign. & 0,985 & $\varnothing$ sign. \\
\hline Geschlecht & 1,580 & $p<0,001$ & 1,644 & $p<0,001$ & 1,621 & $p<0,001$ \\
\hline BMI & 1,153 & $p<0,001$ & 1,163 & $p<0,001$ & 1,166 & $p<0,001$ \\
\hline \multicolumn{7}{|l|}{ Sozialer Status } \\
\hline mittel vs. hoch & 1,019 & $\varnothing$ sign. & 0,921 & $\varnothing$ sign. & 0,878 & $\varnothing$ sign. \\
\hline niedrig vs. hoch & 1,059 & $\varnothing$ sign. & 0,806 & $p=0,051$ & 0,794 & $p=0,05$ \\
\hline Einschränkungen & 1,500 & $p<0,01$ & 1,108 & $\varnothing$ sign. & 1,121 & $\varnothing$ sign. \\
\hline \multicolumn{7}{|l|}{ Schuldurchlauf } \\
\hline $\begin{array}{l}\text { übersprungen vs. } \\
\text { regulär }\end{array}$ & 0,333 & $p=0,067$ & 0,397 & $\varnothing$ sign. & 0,438 & $\varnothing$ sign. \\
\hline $\begin{array}{l}\text { wiederholt vs. regu- } \\
\text { lär }\end{array}$ & 0,773 & $p<0,05$ & 0,604 & $p<0,001$ & 0,580 & $p<0,001$ \\
\hline
\end{tabular}




\begin{tabular}{|c|c|c|c|c|c|c|}
\hline $\begin{array}{c}\text { Körperliche Leis- } \\
\text { tungsfähigkeit }\end{array}$ & & & & & & \\
\hline gut vs. sehr gut & 1,258 & $p<0,05$ & 1,131 & $\varnothing$ sign. & 1,106 & $\varnothing$ sign. \\
\hline mittel vs. sehr gut & 1,461 & $p \leq 0,001$ & 1,087 & $\varnothing$ sign. & 1,114 & $\varnothing$ sign. \\
\hline $\begin{array}{l}\text { nicht besonders gut } \\
\text { vs. sehr gut }\end{array}$ & 1,712 & $p \leq 0,001$ & 0,994 & $\varnothing$ sign. & 1,031 & $\varnothing$ sign. \\
\hline $\begin{array}{l}\text { gar nicht gut vs. } \\
\text { sehr gut }\end{array}$ & 1,189 & $\varnothing$ sign. & 0,333 & $p=0,057$ & 0,345 & $p=0,071$ \\
\hline \multicolumn{7}{|l|}{ TV/Video } \\
\hline $0,5 \mathrm{~h}$ vs. gar nicht & 1,345 & $\varnothing$ sign. & 1,596 & $p=0,089$ & 1,525 & $\varnothing$ sign. \\
\hline $1-2 \mathrm{~h}$ vs. gar nicht & 1,858 & $p<0,01$ & 1,998 & $p<0,01$ & 1,918 & $\mathrm{p}<0,05$. \\
\hline 3-4h vs. gar nicht & 2,007 & $p<0,01$ & 2,095 & $p<0,01$ & 2,037 & $p=0,01$ \\
\hline$>4 \mathrm{~h}$ vs. gar nicht & 1,801 & $p<0,05$ & 1,506 & $\varnothing$ sign. & 1,391 & $\varnothing$ sign. \\
\hline \multicolumn{7}{|l|}{ Computer } \\
\hline $0,5 \mathrm{~h}$ vs. gar nicht & 0,928 & $\varnothing$ sign. & 0,900 & $\varnothing$ sign. & 0,869 & $\varnothing$ sign. \\
\hline 1-2h vs. gar nicht & 1,010 & $\varnothing$ sign. & 0,851 & $\varnothing$ sign. & 0,796 & $p<0,05$ \\
\hline 3-4h vs. gar nicht & 1,211 & $\varnothing$ sign. & 0,859 & $\varnothing$ sign. & 0,814 & $\varnothing$ sign. \\
\hline$>4 \mathrm{~h}$ vs. gar nicht & 1,889 & $p<0,001$ & 1,274 & $\varnothing$ sign. & 1,383 & $p=0,089$ \\
\hline \multicolumn{7}{|l|}{ Bier } \\
\hline $\begin{array}{l}>=1 \mathrm{Glas} / \mathrm{d} \\
\text { vs. gar nicht }\end{array}$ & 2,486 & $p<0,01$ & 1,800 & $p=0,070$ & 1,759 & $\varnothing$ sign. \\
\hline $\begin{array}{l}\text { 5-6 Gläser/Woche } \\
\text { vs. gar nicht }\end{array}$ & 1,519 & $p<0,05$ & 1,203 & $\varnothing$ sign. & 1,161 & $\varnothing$ sign. \\
\hline $\begin{array}{l}\text { 2-4 Gläser/Woche } \\
\text { vs. gar nicht }\end{array}$ & 1,663 & $p<0,001$ & 1,408 & $p<0,05$ & 1,474 & $p<0,05$ \\
\hline $\begin{array}{l}1 \text { Glas/Woche } \\
\text { vs. gar nicht }\end{array}$ & 1,222 & $\varnothing$ sign. & 1,062 & $\varnothing$ sign. & 1,023 & $\varnothing$ sign. \\
\hline $\begin{array}{l}\text { 1-3 Gläser/Monat } \\
\text { vs. gar nicht }\end{array}$ & 1,336 & $p<0,05$ & 1,314 & $p<0,05$ & 1,304 & $p=0,054$ \\
\hline $\begin{array}{l}<1 \text { Glas/Monat } \\
\text { vs. gar nicht }\end{array}$ & 0,726 & $p<0,05$ & 0,708 & $p<0,05$ & 0,752 & $p<0,05$ \\
\hline $\begin{array}{c}\text { Anpassungsgüte } \\
-2 L L\end{array}$ & & & \multicolumn{2}{|c|}{4470,125} & \multicolumn{2}{|c|}{4380,625} \\
\hline
\end{tabular}


Tab. XI Kontrollvariablen und Confounder mit OR \& Signifikanz im univariaten und multivariaten Modell am Bsp. Verhaltensprobleme (Elternangaben)

\begin{tabular}{|c|c|c|c|c|c|c|}
\hline \multirow{2}{*}{$S D Q$} & \multicolumn{2}{|c|}{ univariat } & \multicolumn{2}{|c|}{$\begin{array}{l}\text { multivariat: } \\
\text { Modell } 1\end{array}$} & \multicolumn{2}{|c|}{$\begin{array}{l}\text { multivariat: } \\
\text { Modell 1b }\end{array}$} \\
\hline & $O R$ & Signifikanz & $O R$ & Signifikanz & $O R$ & Signifikanz \\
\hline $\begin{array}{c}\text { Verhaltensprobleme } \\
\text { (Elternangaben) }\end{array}$ & 0,942 & $p<0,05$ & 0,892 & $p<0,001$ & 0,893 & $p<0,001$ \\
\hline Alter & 1,087 & $p<0,001$ & 0,999 & $\varnothing$ sign. & 0,979 & $\varnothing$ sign. \\
\hline Geschlecht & 1,580 & $p<0,001$ & 1,732 & $p<0,001$ & 1,669 & $p<0,001$ \\
\hline$B M I$ & 1,153 & $p<0,001$ & 1,166 & $p<0,001$ & 1,169 & $p<0,001$ \\
\hline \multicolumn{7}{|l|}{ Sozialer Status } \\
\hline mittel vs. hoch & 1,019 & $\varnothing$ sign. & 0,926 & $\varnothing$ sign. & 0,883 & $\varnothing$ sign. \\
\hline niedrig vs. hoch & 1,059 & $\varnothing$ sign. & 0,826 & $p=0,086$ & 0,813 & $p=0,079$ \\
\hline Einschränkungen & 1,500 & $p<0,01$ & 1,108 & $\varnothing$ sign. & 1,089 & $\varnothing$ sign. \\
\hline \multicolumn{7}{|l|}{ Schuldurchlauf } \\
\hline $\begin{array}{c}\text { übersprungen vs. } \\
\text { regulär }\end{array}$ & 0,333 & $p=0,067$ & 0,397 & $\varnothing$ sign. & 0,439 & $\varnothing$ sign. \\
\hline $\begin{array}{c}\text { wiederholt vs. regu- } \\
\text { lär }\end{array}$ & 0,773 & $p<0,05$ & 0,604 & $p<0,001$ & 0,602 & $p<0,001$ \\
\hline \multicolumn{7}{|l|}{$\begin{array}{c}\text { Körperliche Leis- } \\
\text { tungsfähigkeit }\end{array}$} \\
\hline gut vs. sehr gut & 1,258 & $p<0,05$ & 1,131 & $\varnothing$ sign. & 1,080 & $\varnothing$ sign. \\
\hline mittel vs. sehr gut & 1,461 & $p \leq 0,001$ & 1,087 & $\varnothing$ sign. & 1,093 & $\varnothing$ sign. \\
\hline $\begin{array}{l}\text { nicht besonders gut } \\
\text { vs. sehr gut }\end{array}$ & 1,712 & $p \leq 0,001$ & 0,994 & $\varnothing$ sign. & 1,004 & $\varnothing$ sign. \\
\hline $\begin{array}{l}\text { gar nicht gut vs. sehr } \\
\text { gut }\end{array}$ & 1,189 & $\varnothing$ sign. & 0,333 & $p=0,057$ & 0,343 & $p=0,070$ \\
\hline \multicolumn{7}{|l|}{ TV/Video } \\
\hline $0,5 \mathrm{~h}$ vs. gar nicht & 1,345 & $\varnothing$ sign. & 1,596 & $p=0,089$ & 1,503 & $\varnothing$ sign. \\
\hline $1-2 \mathrm{~h}$ vs. gar nicht & 1,858 & $p<0,01$ & 1,998 & $p<0,01$ & 1,876 & $p<0,05$ \\
\hline 3-4h vs. gar nicht & 2,007 & $p<0,01$ & 2,095 & $p<0,01$ & 2,013 & $p<0,05$ \\
\hline$>4 \mathrm{~h}$ vs. gar nicht & 1,801 & $p<0,05$ & 1,506 & $\varnothing$ sign. & 1,380 & $\varnothing$ sign. \\
\hline \multicolumn{7}{|l|}{ Computer } \\
\hline $0,5 \mathrm{~h}$ vs. gar nicht & 0,928 & $\varnothing$ sign. & 0,900 & $\varnothing$ sign. & 0,853 & $\varnothing$ sign. \\
\hline $1-2 \mathrm{~h}$ vs. gar nicht & 1,010 & $\varnothing$ sign. & 0,851 & $\varnothing$ sign. & 0,795 & $p<0,05$ \\
\hline $3-4 \mathrm{~h}$ vs. gar nicht & 1,211 & $\varnothing$ sign. & 0,859 & $\varnothing$ sign. & 0,818 & $\varnothing$ sign. \\
\hline$>4 \mathrm{~h}$ vs. gar nicht & 1,889 & $p<0,001$ & 1,274 & $\varnothing$ sign. & 1,410 & $p=0,072$ \\
\hline
\end{tabular}




\begin{tabular}{|c|c|c|c|c|c|c|}
\hline Bier & & & & & & \\
\hline $\begin{array}{l}>=1 \text { Glas } / d \\
\text { vs. gar nicht }\end{array}$ & 2,486 & $p<0,01$ & 1,800 & $p=0,070$ & 1,884 & $p=0,075$ \\
\hline $\begin{array}{l}\text { 5-6 Gläser/Woche } \\
\text { vs. gar nicht }\end{array}$ & 1,519 & $p<0,05$ & 1,203 & $\varnothing$ sign. & 1,248 & $\varnothing$ sign. \\
\hline $\begin{array}{c}\text { 2-4 Gläser/Woche } \\
\text { vs. gar nicht }\end{array}$ & 1,663 & $p<0,001$ & 1,408 & $p<0,05$ & 1,521 & $p<0,01$ \\
\hline $\begin{array}{l}1 \text { Glas/Woche } \\
\text { vs. gar nicht }\end{array}$ & 1,222 & $\varnothing$ sign. & 1,062 & $\varnothing$ sign. & 1,057 & $\varnothing$ sign. \\
\hline $\begin{array}{c}\text { 1-3 Gläser/Monat } \\
\text { vs. gar nicht }\end{array}$ & 1,336 & $p<0,05$ & 1,314 & $p<0,05$ & 1,344 & $p<0,05$ \\
\hline $\begin{array}{l}<1 \text { Glas/Monat } \\
\text { vs. gar nicht }\end{array}$ & 0,726 & $p<0,05$ & 0,708 & $p<0,05$ & 0,760 & $p=0,053$ \\
\hline $\begin{array}{c}\text { Anpassungsgüte } \\
-2 L L\end{array}$ & & & \multicolumn{2}{|c|}{4470,125} & \multicolumn{2}{|c|}{4370,504} \\
\hline
\end{tabular}

Tab. XII Kontrollvariablen und Confounder mit OR \& Signifikanz im univariaten und multivariaten Modell am Bsp. Hyperaktivität (Elternangaben)

\begin{tabular}{|c|c|c|c|c|c|c|}
\hline \multirow{2}{*}{$S D Q$} & \multicolumn{2}{|c|}{ univariat } & \multicolumn{2}{|c|}{$\begin{array}{c}\text { multivariat: } \\
\text { Modell } 1\end{array}$} & \multicolumn{2}{|c|}{$\begin{array}{l}\text { multivariat: } \\
\text { Modell 1b }\end{array}$} \\
\hline & OR & Signifikanz & OR & Signifikanz & OR & Signifikanz \\
\hline $\begin{array}{l}\text { Hyperaktivität } \\
\text { (Elternangaben) }\end{array}$ & 0,948 & $p<0,01$ & 0,910 & $p<0,001$ & 0,905 & $p<0,001$ \\
\hline Alter & 1,087 & $p<0,001$ & 0,991 & $\varnothing$ sign. & 0,970 & $\varnothing$ sign. \\
\hline Geschlecht & 1,580 & $p<0,001$ & 1,849 & $p<0,001$ & 1,790 & $p<0,001$ \\
\hline$B M I$ & 1,153 & $p<0,001$ & 1,165 & $p<0,001$ & 1,168 & $p<0,001$ \\
\hline \multicolumn{7}{|l|}{ Sozialer Status } \\
\hline mittel vs. hoch & 1,019 & $\varnothing$ sign. & 0,946 & $\varnothing$ sign. & 0,896 & $\varnothing$ sign. \\
\hline niedrig vs. hoch & 1,059 & $\varnothing$ sign. & 0,842 & $\varnothing$ sign. & 0,825 & $\varnothing$ sign. \\
\hline Einschränkungen & 1,500 & $p<0,01$ & 1,108 & $\varnothing$ sign. & 1,109 & $\varnothing$ sign. \\
\hline \multicolumn{7}{|l|}{ Schuldurchlauf } \\
\hline $\begin{array}{l}\text { übersprungen vs. } \\
\text { regulär }\end{array}$ & 0,333 & $p=0,067$ & 0,397 & $\varnothing$ sign. & 0,433 & $\varnothing$ sign. \\
\hline $\begin{array}{l}\text { wiederholt vs. regu- } \\
\text { lär }\end{array}$ & 0,773 & $p<0,05$ & 0,604 & $p<0,001$ & 0,633 & $p<0,001$ \\
\hline
\end{tabular}




\begin{tabular}{|c|c|c|c|c|c|c|}
\hline $\begin{array}{c}\text { Körperliche Leis- } \\
\text { tungsfähigkeit }\end{array}$ & & & & & & \\
\hline gut vs. sehr gut & 1,258 & $p<0,05$ & 1,131 & $\varnothing$ sign. & 1,109 & $\varnothing$ sign. \\
\hline mittel vs. sehr gut & 1,461 & $p \leq 0,001$ & 1,087 & $\varnothing$ sign. & 1,106 & $\varnothing$ sign. \\
\hline $\begin{array}{l}\text { nicht besonders gut } \\
\text { vs. sehr gut }\end{array}$ & 1,712 & $p \leq 0,001$ & 0,994 & $\varnothing$ sign. & 1,029 & $\varnothing$ sign. \\
\hline $\begin{array}{l}\text { gar nicht gut vs. } \\
\text { sehr gut }\end{array}$ & 1,189 & $\varnothing$ sign. & 0,333 & $p=0,057$ & 0,337 & $p=0,065$ \\
\hline \multicolumn{7}{|l|}{ TV/Video } \\
\hline $0,5 \mathrm{~h}$ vs. gar nicht & 1,345 & $\varnothing$ sign. & 1,596 & $p=0,089$ & 1,529 & $\varnothing$ sign. \\
\hline $1-2 \mathrm{~h}$ vs. gar nicht & 1,858 & $p<0,01$ & 1,998 & $p<0,01$ & 1,950 & $p<0,05$ \\
\hline 3-4h vs. gar nicht & 2,007 & $p<0,01$ & 2,095 & $p<0,01$ & 2,072 & $p<0,01$ \\
\hline$>4 \mathrm{~h}$ vs. gar nicht & 1,801 & $p<0,05$ & 1,506 & $\varnothing$ sign. & 1,448 & $\varnothing$ sign. \\
\hline \multicolumn{7}{|l|}{ Computer } \\
\hline $0,5 \mathrm{~h}$ vs. gar nicht & 0,928 & $\varnothing$ sign. & 0,900 & $\varnothing$ sign. & 0,869 & $\varnothing$ sign. \\
\hline 1-2h vs. gar nicht & 1,010 & $\varnothing$ sign. & 0,851 & $\varnothing$ sign. & 0,804 & $p=0,059$ \\
\hline 3-4h vs. gar nicht & 1,211 & $\varnothing$ sign. & 0,859 & $\varnothing$ sign. & 0,821 & $\varnothing$ sign. \\
\hline$>4 \mathrm{~h}$ vs. gar nicht & 1,889 & $p<0,001$ & 1,274 & $\varnothing$ sign. & 1,454 & $p=0,051$ \\
\hline \multicolumn{7}{|l|}{ Bier } \\
\hline $\begin{array}{l}>=1 \mathrm{Glas} / \mathrm{d} \\
\text { vs. gar nicht }\end{array}$ & 2,486 & $p<0,01$ & 1,800 & $p=0,070$ & 1,913 & $p=0,068$. \\
\hline $\begin{array}{l}\text { 5-6 Gläser/Woche } \\
\text { vs. gar nicht }\end{array}$ & 1,519 & $p<0,05$ & 1,203 & $\varnothing$ sign. & 1,205 & $\varnothing$ sign. \\
\hline $\begin{array}{l}\text { 2-4 Gläser/Woche } \\
\text { vs. gar nicht }\end{array}$ & 1,663 & $p<0,001$ & 1,408 & $p<0,05$ & 1,508 & $p<0,01$ \\
\hline $\begin{array}{l}1 \text { Glas/Woche } \\
\text { vs. gar nicht }\end{array}$ & 1,222 & $\varnothing$ sign. & 1,062 & $\varnothing$ sign. & 1,062 & $\varnothing$ sign. \\
\hline $\begin{array}{l}\text { 1-3 Gläser/Monat } \\
\text { vs. gar nicht }\end{array}$ & 1,336 & $p<0,05$ & 1,314 & $p<0,05$ & 1,330 & $p<0,05$ \\
\hline $\begin{array}{l}<1 \text { Glas/Monat } \\
\text { vs. gar nicht }\end{array}$ & 0,726 & $p<0,05$ & 0,708 & $p<0,05$ & 0,755 & $p<0,05$ \\
\hline $\begin{array}{c}\text { Anpassungsgüte } \\
-2 L L\end{array}$ & & & \multicolumn{2}{|c|}{4470,125} & \multicolumn{2}{|c|}{4354,273} \\
\hline
\end{tabular}


Tab. XIII Kontrollvariablen und Confounder mit OR \& Signifikanz im univariaten und multivariaten Modell am Bsp. des Gesamtproblemwerts (Elternangaben)

\begin{tabular}{|c|c|c|c|c|c|c|}
\hline \multirow{2}{*}{$S D Q$} & \multicolumn{2}{|c|}{ univariat } & \multicolumn{2}{|c|}{$\begin{array}{c}\text { multivariat: } \\
\text { Modell } 1\end{array}$} & \multicolumn{2}{|c|}{$\begin{array}{l}\text { multivariat: } \\
\text { Modell 1b }\end{array}$} \\
\hline & $O R$ & Signifikanz & $O R$ & Signifikanz & $O R$ & Signifikanz \\
\hline $\begin{array}{c}\text { Gesamtproblemwert } \\
\text { (Elternangaben) }\end{array}$ & 0,977 & $p<0,01$ & 0,958 & $p<0,001$ & 0,958 & $p<0,001$ \\
\hline Alter & 1,087 & $p<0,001$ & 0,989 & $\varnothing$ sign. & 0,972 & $\varnothing$ sign. \\
\hline Geschlecht & 1,580 & $p<0,001$ & 1,772 & $p<0,001$ & 1,730 & $p<0,001$ \\
\hline$B M I$ & 1,153 & $p<0,001$ & 1,169 & $p<0,001$ & 1,169 & $p<0,001$ \\
\hline \multicolumn{7}{|l|}{ Sozialer Status } \\
\hline mittel vs. hoch & 1,019 & $\varnothing$ sign. & 0,953 & $\varnothing$ sign. & 0,904 & $\varnothing$ sign. \\
\hline niedrig vs. hoch & 1,059 & $\varnothing$ sign. & 0,878 & $\varnothing$ sign. & 0,857 & $\varnothing$ sign. \\
\hline Einschränkungen & 1,500 & $p<0,01$ & 1,108 & $\varnothing$ sign. & 1,198 & $\varnothing$ sign. \\
\hline \multicolumn{7}{|l|}{ Schuldurchlauf } \\
\hline $\begin{array}{l}\text { übersprungen vs. } \\
\text { regulär }\end{array}$ & 0,333 & $p=0,067$ & 0,397 & $\varnothing$ sign. & 0,457 & $\varnothing$ sign. \\
\hline $\begin{array}{c}\text { wiederholt vs. regu- } \\
\text { lär }\end{array}$ & 0,773 & $p<0,05$ & 0,604 & $p<0,001$ & 0,628 & $p<0,001$ \\
\hline \multicolumn{7}{|l|}{$\begin{array}{c}\text { Körperliche Leis- } \\
\text { tungsfähigkeit }\end{array}$} \\
\hline gut vs. sehr gut & 1,258 & $p<0,05$ & 1,131 & $\varnothing$ sign. & 1,115 & $\varnothing$ sign. \\
\hline mittel vs. sehr gut & 1,461 & $p \leq 0,001$ & 1,087 & $\varnothing$ sign. & 1,159 & $\varnothing$ sign. \\
\hline $\begin{array}{c}\text { nicht besonders gut } \\
\text { vs. sehr gut }\end{array}$ & 1,712 & $p \leq 0,001$ & 0,994 & $\varnothing$ sign. & 1,098 & $\varnothing$ sign. \\
\hline $\begin{array}{c}\text { gar nicht gut vs. sehr } \\
\text { gut }\end{array}$ & 1,189 & $\varnothing$ sign. & 0,333 & $p=0,057$ & 0,368 & $p=0,092$ \\
\hline \multicolumn{7}{|l|}{ TV/Video } \\
\hline $0,5 \mathrm{~h}$ vs. gar nicht & 1,345 & $\varnothing$ sign. & 1,596 & $p=0,089$ & 1,494 & $\varnothing$ sign. \\
\hline 1-2h vs. gar nicht & 1,858 & $p<0,01$ & 1,998 & $p<0,01$ & 1,895 & $p<0,05$ \\
\hline 3-4h vs. gar nicht & 2,007 & $p<0,01$ & 2,095 & $p<0,01$ & 2,040 & $p<0,05$ \\
\hline$>4 \mathrm{~h}$ vs. gar nicht & 1,801 & $p<0,05$ & 1,506 & $\varnothing$ sign. & 1,423 & $\varnothing$ sign. \\
\hline \multicolumn{7}{|l|}{ Computer } \\
\hline $0,5 \mathrm{~h}$ vs. gar nicht & 0,928 & $\varnothing$ sign. & 0,900 & $\varnothing$ sign. & 0,863 & $\varnothing$ sign. \\
\hline 1-2h vs. gar nicht & 1,010 & $\varnothing$ sign. & 0,851 & $\varnothing$ sign. & 0,807 & $p=0,065$ \\
\hline 3-4h vs. gar nicht & 1,211 & $\varnothing$ sign. & 0,859 & $\varnothing$ sign. & 0,825 & $\varnothing$ sign. \\
\hline$>4 \mathrm{~h}$ vs. gar nicht & 1,889 & $p<0,001$ & 1,274 & $\varnothing$ sign. & 1,436 & $p=0,059$ \\
\hline \multicolumn{7}{|l|}{ Bier } \\
\hline$>=1 \mathrm{Glas} / \mathrm{d}$ & 2,486 & $p<0,01$ & 1,800 & $p=0,070$ & 1,832 & $p=0,088$ \\
\hline
\end{tabular}




\begin{tabular}{|c|c|c|c|c|c|c|}
\hline vs. gar nicht & & & & & & \\
\hline $\begin{array}{c}5-6 \text { Gläser/Woche } \\
\text { vs. gar nicht }\end{array}$ & 1,519 & $p<0,05$ & 1,203 & $\varnothing$ sign. & 1,175 & $\varnothing$ sign. \\
\hline $\begin{array}{c}\text { 2-4 Gläser/Woche } \\
\text { vs. gar nicht }\end{array}$ & 1,663 & $p<0,001$ & 1,408 & $p<0,05$. & 1,484 & $p<0,05$ \\
\hline $\begin{array}{c}\text { 1 Glas/Woche } \\
\text { vs. gar nicht }\end{array}$ & 1,222 & $\varnothing$ sign. & 1,062 & $\varnothing$ sign. & 1,029 & $\varnothing$ sign. \\
\hline $\begin{array}{c}1-3 \text { Gläser/Monat } \\
\text { vs. gar nicht }\end{array}$ & 1,336 & $p<0,05$ & 1,314 & $p<0,05$. & 1,326 & $p<0,05$ \\
\hline $\begin{array}{c}<1 \text { Glas/Monat } \\
\text { vs. gar nicht }\end{array}$ & 0,726 & $p<0,05$ & 0,708 & $p<0,05$ & 0,756 & $p<0,05$ \\
\hline $\begin{array}{c}\text { Anpassungsgüte } \\
-2 L L\end{array}$ & & 4470,125 & 4347,939 \\
\hline
\end{tabular}




\section{Literaturverzeichnis}

Abeyta IM, Tuitt NR, Byers TE, Sauaia A (2012): Effect of community affluence on the association between individual socioeconomic status and cardiovascular disease risk factors, colorado, 20072008. Prev Chronic Dis $\underline{9}$, E115

Adam G, Bela A, Koo E, Szekely JI (1963): Carotid afferentation and higher nervous activity.II. Conditioned reflexes of rats deprived of their carotid innervation. Acta Physiol Acad Sci Hung $\underline{23}$, 339-342

Adams HR, Szilagyi PG, Gebhardt L, Lande MB (2010): Learning and attention problems among children with pediatric primary hypertension. Pediatrics $\underline{126}$, e1425-1429

Anderson NB, McNeilly M (1991): Age,gender,and ethnicity as variables in psychophysiological assessment: Sociodemographics in context. Psychol Assess $\underline{3}$, 376-384

Apkarian AV, Jyvasjarvi E, Kniffki KD, Mengel MK, Stiefenhofer A (1989): Activation of carotid sinus baroreceptors reduces pain sensations evoked by electrical and cold stimulation of human teeth. Proc Finn Dent Soc $\underline{85}, 409-413$

Arslantas D, Ayranci U, Unsal A, Tozun M (2008): Prevalence of hypertension among individuals aged 50 years and over and its impact on health related quality of life in a semi-rural area of western Turkey. Chin Med J (Engl) 121, 1524-1531

Assadi F (2008): Relation of left ventricular hypertrophy to microalbuminuria and C-reactive protein in children and adolescents with essential hypertension. Pediatr Cardiol $\underline{29}$, 580-584

American Psychiatric Association: Diagnostic and Statistical Manual of Mental Disorders. 4. Auflage; Washington DC 1994

Babbs CF (2012): Oscillometric measurement of systolic and diastolic blood pressures validated in a physiologic mathematical model. Biomed Eng Online 11, 56

Bakris GL, Nadim MK, Haller H, Lovett EG, Schafer JE, Bisognano JD (2012): Baroreflex activation therapy provides durable benefit in patients with resistant hypertension: results of long-term follow-up in the Rheos Pivotal Trial. J Am Soc Hypertens $\underline{6}$, 152-158

Bancalari R, Diaz C, Martinez-Aguayo A, Aglony M, Zamorano J, Cerda V, Fernandez M, Garbin F, Cavada G, Valenzuela M, et al. (2011): Prevalence of hypertension in school age children and its association with obesity. Rev Med Chil 139, 872-879 
Bao W, Threefoot SA, Srinivasan SR, Berenson GS (1995): Essential hypertension predicted by tracking of elevated blood pressure from childhood to adulthood: the Bogalusa Heart Study. Am J Hypertens $\underline{8}, 657-665$

Bardage C, Isacson DG (2000): Self-reported side-effects of antihypertensive drugs: an epidemiological study on prevalence and impact on health-state utility. Blood Press $\underline{9}, 328-334$

Bardage C, Isacson DG (2001): Hypertension and health-related quality of life. an epidemiological study in Sweden. J Clin Epidemiol 54, 172-181

Barger SD, Muldoon MF (2006): Hypertension labelling was associated with poorer self-rated health in the Third US National Health and Nutrition Examination Survey. J Hum Hypertens $\underline{20}$, 117-123

Battersby C, Hartley K, Fletcher AF, Markowe HJ, Styles W, Sapper H, Bulpitt CJ (1995): Quality of life in treated hypertension: a case-control community based study. J Hum Hypertens $\underline{9}$, 981-986

Bayer O, Neuhauser H, von Kries R (2009): Sleep duration and blood pressure in children: a crosssectional study. J Hypertens 27, 1789-1793

Beatty DL, Matthews KA (2009): Unfair treatment and trait anger in relation to nighttime ambulatory blood pressure in African American and white adolescents. Psychosom Med $\underline{71}, 813-$ 820

Berenson GS, Srinivasan SR, Bao W, Newman WP, Tracy RE, Wattigney WA (1998): Association between multiple cardiovascular risk factors and atherosclerosis in children and young adults. The Bogalusa Heart Study. N Engl J Med $\underline{338}$, 1650-1656

Bethell CD, Read D, Stein RE, Blumberg SJ, Wells N, Newacheck PW (2002): Identifying children with special health care needs: development and evaluation of a short screening instrument. Ambul Pediatr $\underline{2}, 38-48$

Bettge S, Ravens-Sieberer U (2003): Schutzfaktoren für die psychische Gesundheit von Kindern und Jugendlichen - empirische Ergebnisse zur Validierung eines Konzepts. Gesundheitswesen $\underline{65}$, $167-172$

Bloom JR, Monterossa S (1981): Hypertension labeling and sense of well-being. Am J Public Health $\underline{71}, 1228-1232$

Bonvallet M, Dell P, Hiebel G (1953): The carotid sinus and cerebral electrical activity. C R Seances Soc Biol Fil 147, 1166-1169

Bremner AD (2002): Antihypertensive medication and quality of life--silent treatment of a silent killer? Cardiovasc Drugs Ther 16, 353-364 
Bühl A: PASW 18 Einführung in die moderne Datenanalyse. 12. Auflage; Pearson Studium, München 2010

Bullinger M, von Mackensen S, Kirchberger I (1994): KINDL-ein Fragebogen zur Erfassung der gesundheitsbezogenen Lebensqualität von Kindern. Z Gesundheitspsychol 2, 64-77

Carroll D, Ginty AT, Painter RC, Roseboom TJ, Phillips AC, de Rooij SR (2012): Systolic blood pressure reactions to acute stress are associated with future hypertension status in the Dutch Famine Birth Cohort Study. Int J Psychophysiol 85, 270-273

Chen LJ, Fox KR, Haase A, Wang JM (2006): Obesity, fitness and health in Taiwanese children and adolescents. Eur J Clin Nutr $\underline{60}, 1367-1375$

Chiolero A, Bovet P, Paradis G, Paccaud F (2007): Has blood pressure increased in children in response to the obesity epidemic? Pediatrics $\underline{119}, 544-553$

Chobanian AV, Bakris GL, Black HR, Cushman WC, Green LA, Izzo JL, Jones DW, Materson BJ, Oparil S, Wright JT, et al. (2003): Seventh report of the Joint National Committee on Prevention, Detection, Evaluation, and Treatment of High Blood Pressure. Hypertension 42, 1206-1252

Coelho R, Hughes AM, da Fonseca AF, Bond MR (1989): Essential hypertension: the relationship of psychological factors to the severity of hypertension. J Psychosom Res $\underline{33}, 187-196$

Daniels SR, Loggie JM, Khoury P, Kimball TR (1998): Left ventricular geometry and severe left ventricular hypertrophy in children and adolescents with essential hypertension. Circulation $\underline{97}$, 1907-1911

Dimenas ES, Wiklund IK, Dahlof CG, Lindvall KG, Olofsson BK, De Faire UH (1989): Differences in the subjective well-being and symptoms of normotensives, borderline hypertensives and hypertensives. J Hypertens $\underline{7}, 885-890$

Din-Dzietham R, Liu Y, Bielo MV, Shamsa F (2007): High blood pressure trends in children and adolescents in national surveys, 1963 to 2002 . Circulation $\underline{116}, 1488-1496$

Donald CA, Ware JE (1984): The measurement of social support. Res Community Mental Health $\underline{4}$, 325-370

Durrani AM, Waseem F (2011): Blood pressure distribution and its relation to anthropometric measurements among school children in Aligarh. Indian J Public Health $\underline{55}$, 121-124

Dworkin BR, Filewich RJ, Miller NE, Craigmyle N, Pickering TG (1979): Baroreceptor activation reduces reactivity to noxious stimulation: implications for hypertension. Science $\underline{205}, 1299-1301$ 
Dworkin BR, Elbert T, Rau H, Birbaumer N, Pauli P, Droste C, Brunia CH (1994): Central effects of baroreceptor activation in humans: attenuation of skeletal reflexes and pain perception. Proc Natl Acad Sci U S A 91, 6329-6333

Elbert T, Dworkin BR, Rau H, Pauli P, Birbaumer N, Droste C, Brunia CH (1994): Sensory effects of baroreceptor activation and perceived stress together predict long-term blood pressure elevations. Int J Behav Med 1, 215-228

Erhart M, Hölling H, Bettge S, Ravens-Sieberer U, Schlack R (2007): Der Kinder- und Jugendgesundheitssurvey (KiGGS): Risiken und Ressourcen für die psychische Entwicklung von Kindern und Jugendlichen. Bundesgesundheitsblatt Gesundheitsforschung Gesundheitsschutz $\underline{50}$, 800-809

Erickson SR, Williams BC, Gruppen LD (2001): Perceived symptoms and health-related quality of life reported by uncomplicated hypertensive patients compared to normal controls. J Hum Hypertens $\underline{15}$, 539-548

Erickson SR, Williams BC, Gruppen LD (2004): Relationship between symptoms and health-related quality of life in patients treated for hypertension. Pharmacotherapy $24,344-350$

Ewart CK, Kolodner KB (1994): Negative affect, gender, and expressive style predict elevated ambulatory blood pressure in adolescents. J Pers Soc Psychol 66, 596-605

Ewart CK, Jorgensen RS, Kolodner KB (1998): Sociotropic cognition moderates blood pressure response to interpersonal stress in high-risk adolescent girls. Int J Psychophysiol 28, 131-142

Ewart CK, Elder GJ, Smyth JM, Sliwinski MJ, Jorgensen RS (2011): Do agonistic motives matter more than anger? Three studies of cardiovascular risk in adolescents. Health Psychol $\underline{30}, 510-524$

Fahrmeir L, Künstler R, Pigeot I, Tutz G: Statistik Der Weg zur Datenanalyse. 5. Auflage; Springer, Berlin 2004

Falkner B, Gidding SS, Portman R, Rosner B (2008): Blood pressure variability and classification of prehypertension and hypertension in adolescence. Pediatrics $\underline{122}$, 238-242

Falkner B, Lurbe E, Schaefer F (2010): High blood pressure in children: Clinical and health policy implications. J Clin Hypertens (Greenwich) 12, 261-276

Flechtner-Mors M, Thamm M, Rosario AS, Goldapp C, Hoffmeister U, Mann R, Bullinger M, van Egmond-Frohlich A, Ravens-Sieberer U, Reinehr T, et al. (2011): Hypertension, dyslipoproteinemia and $\mathrm{BMI}$-category characterise the cardiovascular risk in overweight or obese children and adolescents: data of the BZgA-observational study (EvAKu-J-project) and the KiGGS-study. Klin Padiatr 223, 445-449 
Flouris AD, Faught BE, Klentrou P (2008): Cardiovascular disease risk in adolescent smokers: evidence of a 'smoker lifestyle'. J Child Health Care 12, 221-231

France CR (1999): Decreased pain perception and risk for hypertension: considering a common physiological mechanism. Psychophysiology 36, 683-692

Gasperin D, Netuveli G, Dias-da-Costa JS, Pattussi MP (2009): Effect of psychological stress on blood pressure increase: a meta-analysis of cohort studies. Cad Saude Publica 25, 715-726

Gillman MW, Cook NR (1995): Blood pressure measurement in childhood epidemiological studies. Circulation 92, 1049-1057

Goldfield GS, Kenny GP, Hadjiyannakis S, Phillips P, Alberga AS, Saunders TJ, Tremblay MS, Malcolm J, Prud'homme D, Gougeon R, et al. (2011): Video game playing is independently associated with blood pressure and lipids in overweight and obese adolescents. PLoS One $\underline{6}$, e26643

Goodman R (1997): The Strength and Difficulties Questionnaire: A research note. J Child Psychol Psychiatry 38, 581-586

Goodman R, Scott S (1999): Comparing the Strengths and Difficulties Questionnaire and the Child Behavior Checklist: Is small beautiful? J Abnorm Child Psychol 27, 17-24

Goodman R, Meltzer H, Bailey V (1998): The Strengths and Difficulties Questionnaire: A pilot study on the validity of the self-report version. Eur Child \& Adolesc Psychiatry $\underline{Z}, 125-130$

Grob A, Lüthi R, Kaiser FG, Flammer A, Mackinnon A, Wearing AJ (1991): Berner Fragebogen zum Wohlbefinden Jugendlicher (BFB). Diagn $\underline{37}$, 66-75

Haas GM, Liepold E, Schwandt P (2012 a): Metabolic risk factors, leisure time physical activity, and nutrition in German children and adolescents. Cholesterol 2012, 370850

Haas GM, Bertsch T, Schwandt P (2012 b): Trends of components of the metabolic syndrome in German first graders throughout 10 years: The PEP Family Heart Study. Cholesterol 2012, 231962

Hansen ML, Gunn PW, Kaelber DC (2007): Underdiagnosis of hypertension in children and adolescents. JAMA $\underline{298}, 874-879$

Hayes DK, Denny CH, Keenan NL, Croft JB, Greenlund KJ (2008): Health-related quality of life and hypertension status, awareness, treatment, and control: National Health and Nutrition Examination Survey, 2001--2004. J Hypertens 26, 641-647 
Hölling H, Erhart M, Ravens-Sieberer U, Schlack R (2007 a): Verhaltensauffälligkeiten bei Kindern und Jugendlichen. Bundesgesundheitsblatt Gesundheitsforschung Gesundheitsschutz $\underline{50}$, 784-793

Hölling H, Kamtsiuris P, Lange M, Thierfelder W, Thamm M, Schlack R (2007 b): Der Kinder- und Jugendgesundheitssurvey (KiGGS): Studienmanagement und Durchführung der Feldarbeit. Bundesgesundheitsblatt Gesundheitsforschung Gesundheitsschutz 50, 557-566

Irvine MJ, Garner DM, Olmsted MP, Logan AG (1989): Personality differences between hypertensive and normotensive individuals: influence of knowledge of hypertension status. Psychosom Med 51, 537-549

Javaheri S, Storfer-Isser A, Rosen CL, Redline S (2008): Sleep quality and elevated blood pressure in adolescents. Circulation 118, 1034-1040

Jenkins CD, Hurst MW, Rose RM: Biomedical and psychosocial predictors of hypertension in air traffic controllers; in: Stress and Anxiety; Spielberger A and Sarason I; 9; Hemisphere Publishing Corporation, Washington DC 1985, 231-238

Jöckel KH, Babitsch B, Bellach BM: Messung und Quantifizierung soziodemographischer Merkmale in epidemiologischen Studien. RKI-Schriften MMV Medizin Verlag, München 1998

Jorgensen RS, Maisto SA (2008): Alcohol consumption and prehypertension: an investigation of university youth. Behav Med $\underline{34}, 21-28$

Kamtsiuris P, Bergmann KE, Dippelhofer A, Hölling H, Kurth BM, Thefeld W (2002): Der Pretest des Kinder- und Jugendgesundheitssurveys: Methodische Aspekte und Durchführung. Gesundheitswesen 64 (Sonderheft 1), 36-42

Kamtsiuris P, Lange M, Schaffrath Rosario A (2007): Der Kinder- und Jugendgesundheitssurvey (KiGGS): Stichprobendesign, Response und Nonresponse-Analyse. Bundesgesundheitsblatt Gesundheitsforschung Gesundheitsschutz 50, 547-556

Katona E, Zrinyi M, Komonyi E, Lengyel S, Paragh G, Zatik J, Fulesdi B, Pall D (2011): Factors influencing adolescent blood pressure: the Debrecen Hypertension Study. Kidney Blood Press Res 34, 188-195

Kern R, Rasky E, Noack RH: Indikatoren für Gesundheitsförderung in der Volksschule. Forschungsbericht 95/1. Karl-Franzens-Universität, Graz 1995

Khang YH, Lynch JW (2011): Exploring determinants of secular decreases in childhood blood pressure and hypertension. Circulation 124, 397-405 
Khosravi A, Ramezani MA, Toghianifar N, Rabiei K, Jahandideh M, Yousofi A (2010): Association between hypertension and quality of life in a sample of Iranian adults. Acta Cardiol $\underline{65}$, 425-430

Kjellgren KI, Ahlner J, Dahlof B, Gill H, Hedner T, Saljo R (1998): Perceived symptoms amongst hypertensive patients in routine clinical practice- a population-based study. J Intern Med $\underline{244}$ 325-332

Klasen H, Woerner W, Wolke D, Meyer R, Overmeyer S, Kaschnitz W, Rothenberger A, Goodmann $R$ (2000): Comparing the German versions of the Strength and Difficulties Questionnaire (SDQDeu) and the Child Behavior Checklist. Eur Child Adolesc Psychiatry 9 , 271-276

Klimm HD, Reuter-Kuhn I (1994): Prevalence of hypertension in childhood. A study of 21 family physician practices. Fortschr Med 112, 49-52

Klinkenberg S, Majoie HJ, van der Heijden MM, Rijkers K, Leenen L, Aldenkamp AP (2012): Vagus nerve stimulation has a positive effect on mood in patients with refractory epilepsy. Clin Neurol Neurosurg $\underline{114}, 336-340$

Klocek M, Kawecka-Jaszcz K (2003): Quality of life in patients with essential arterial hypertension. Part I: The effect of socio-demographic factors Przegl Lek $\underline{60}, 92-100$

Kollias A, Antonodimitrakis P, Grammatikos E, Chatziantonakis N, Grammatikos EE, Stergiou GS (2009): Trends in high blood pressure prevalence in Greek adolescents. J Hum Hypertens $\underline{23}$, 385390

Korhonen PE, Kivelä S-L, Kautiainen H, Järvenpää S, Kantola I (2011): Health-related quality of life and awareness of hypertension. J Hypertens $\underline{29}$, 2070-2074

Kosti RI, Panagiotakos DB (2006): The epidemic of obesity in children and adolescents in the world. Cent Eur J Public Health 14, 151-159

Kurth BM (2007): Der Kinder- und Jugendgesundheitssurvey (KiGGS): Ein Überblick über Planung, Durchführung und Ergebnisse unter Berücksichtigung von Aspekten eines Qualitätsmanagements. Bundesgesundheitsblatt Gesundheitsforschung Gesundheitsschutz 50, 533-546

Kurth BM, Bergmann KE, Hölling H, Kahl K, Kamtsiuris P, Thefeld W (2002): Der bundesweite Kinder- und Jugendgesundheitssurvey. Das Gesamtkonzept. Gesundheitswesen $\underline{64}$ (Sonderheft 1) 3-11

Lahad A, Yodfat Y (1993): Impact of comorbidity on well-being in hypertension: case control study. J Hum Hypertens $\underline{Z}, 611-614$ 
Lampert T, Kroll LE: Die Messung des sozioökonomischen Status in sozialepidemiologischen Studien. VS Verlag für Sozialwissenschaften, Wiesbaden 2006

Lampert T, Schenk L, Stolzenberg H (2002): Konzeptualisierung und Operationalisierung sozialer Ungleichheit im Kinder- und Jugendgesundheitssurvey. Gesundheitswesen 64 (Sonderheft 1), 4852

Lande MB, Kaczorowski JM, Auinger P, Schwartz GJ, Weitzman M (2003): Elevated blood pressure and decreased cognitive function among school-age children and adolescents in the United States. J Pediatr. $\underline{143}, 720-724$

Lande MB, Adams H, Falkner B, Waldstein SR, Schwartz GJ, Szilagyi PG, Wang H, Palumbo D (2009): Parental assessments of internalizing and externalizing behavior and executive function in children with primary hypertension. J Pediatr 154, 207-212

Lange M, Kamtsiuris P, Lange C, Schaffrath Rosario A, Stolzenberg H, Lampert T (2007): Messung soziodemographischer Merkmale im Kinder- und Jugendgesundheitssurvey (KiGGS) und ihre Bedeutung am Beispiel der Einschätzung des allgemeinen Gesundheitszustands. Bundesgesundheitsblatt Gesundheitsforschung Gesundheitsschutz 50, 578-589

Li W, Liu L, Puente JG, Li Y, Jiang X, Jin S, Ma H, Kong L, Ma L, He X, et al. (2005): Hypertension and health-related quality of life: an epidemiological study in patients attending hospital clinics in China. J Hypertens $\underline{23}$, 1667-1676

Linden W, Feuerstein M (1983): Essential hypertension and social coping behavior: experimental findings. J Human Stress $\underline{9}$, 22-31

Lohmeier TE, Irwin ED, Rossing MA, Serdar DJ, Kieval RS (2004): Prolonged activation of the baroreflex produces sustained hypotension. Hypertension $\underline{43}, 306-311$

Lohmeier TE, Dwyer TM, Irwin ED, Rossing MA, Kieval RS (2007): Prolonged activation of the baroreflex abolishes obesity-induced hypertension. Hypertension $\underline{49}$, 1307-1314

Luma GB, Spiotta RT (2006): Hypertension in children and adolescents. Am Fam Physician $\underline{73}$, 1558-1568

Lurbe E, Cifkova R, Cruickshank JK, Dillon MJ, Ferreira I, Invitti C, Kuznetsova T, Laurent S, Mancia $\mathrm{G}$, Morales-Olivas F, et al. (2009): Management of high blood pressure in children and adolescents: recommendations of the European Society of Hypertension. J Hypertens $\underline{27}$, 17191742

Maixner W (1991): Interactions between cardiovascular and pain modulatory systems:physiological and pathophysiological implications. J Cardiovasc Electrophysiol $\underline{2}, 3-12$ 
Makris A, Seferou M, Papadopoulos DP (2010): Resistant hypertension workup and approach to treatment. Int J Hypertens 2011, 598694

Malatesta-Muncher R, Mitsnefes MM (2012): Management of blood pressure in children. Curr Opin Nephrol Hypertens 21, $318-322$

Maldonado J, Pereira T, Fernandes R, Santos R, Carvalho M (2011): An approach of hypertension prevalence in a sample of 5381 Portuguese children and adolescents. The AVELEIRA registry. "Hypertension in children". Blood Press 20, 153-157

Mann SJ (2012): Psychosomatic research in hypertension: the lack of impact of decades of research and new directions to consider. J Clin Hypertens (Greenwich) 14, 657-664

Mayes SD, Calhoun SL, Crowell EW (2000): Learning disabilities and ADHD: overlapping spectrumn disorders. J Learn Disabil $\underline{33}$, 417-424

McNiece KL, Poffenbarger TS, Turner JL, Franco KD, Sorof JM, Portman RJ (2007): Prevalence of hypertension and pre-hypertension among adolescents. J Pediatr $\underline{150}$, 640-644

Mena-Martin FJ, Martin-Escudero JC, Simal-Blanco F, Carretero-Ares JL, Arzua-Mouronte D, Herreros-Fernandez V (2003): Health-related quality of life of subjects with known and unknown hypertension: results from the population-based Hortega study. J Hypertens $\underline{21}, 1283-1289$

Milligan RA, Burke V, Dunbar DL, Spencer M, Balde E, Beilin LJ, Gracey MP (1997): Associations between lifestyle and cardiovascular risk factors in 18-year-old Australians. J Adolesc Health 21, 186-195

Mindray: Produktinformationsblatt des Blutdruckmessgeräts: Accutorr Plus Service Manual. Datascope Corporation, USA 2007

Mohr P, Rodriguez M, Slavickova A, Hanka J (2011): The application of vagus nerve stimulation and deep brain stimulation in depression. Neuropsychobiology $\underline{64}, 170-181$

Munger RG, Prineas RJ, Gomez-Marin O (1988): Persistent elevation of blood pressure among children with a family history of hypertension: the Minneapolis Children's Blood Pressure Study. J Hypertens $\underline{6}$, 647-653

Muntner P, He J, Cutler JA, Wildman RP, Whelton PK (2004): Trends in blood pressure among children and adolescents. JAMA 291, 2107-2113

Neuhauser H, Thamm M (2007): Blutdruckmessung im Kinder- und Jugendgesundheitssurvey (KiGGS). Bundesgesundheitsblatt Gesundheitsforschung Gesundheitsschutz $\underline{50}$, 728-735 
Neuhauser HK, Rosario AS, Thamm M, Ellert U (2009): Prevalence of children with blood pressure measurements exceeding adult cutoffs for optimal blood pressure in Germany. Eur J Cardiovasc Prev Rehabil 16, 195-200

Neuhauser HK, Thamm M, Ellert U, Hense HW, Rosario AS (2011): Blood pressure percentiles by age and height from nonoverweight children and adolescents in Germany. Pediatrics $\underline{127}$, e978988

$\mathrm{Ng}$, Sica DA, Frishman WH (2011): Rheos: an implantable carotid sinus stimulation device for the nonpharmacologic treatment of resistant hypertension. Cardiol Rev $\underline{19}, 52-57$

NHBPEP (2004): The Fourth Report on the Diagnosis, Evaluation, and Treatment of High Blood Pressure in Children and Adolescents. Pediatrics $\underline{114}$, 555-576

Nielsen GA, Andersen LB (2003): The association between high blood pressure, physical fitness, and body mass index in adolescents. Prev Med 36, 229-234

Nyklicek I, Vingerhoets JJ, Van Heck GL (1996): Hypertension and objective and self-reported stressor exposure: a review. J Psychosom Res $\underline{40}$, 585-601

O'Brien E, Waeber B, Parati G, Staessen J, Myers MG (2001): Blood pressure measuring devices: recommendations of the European Society of Hypertension. BMJ 322, 531-536

World Health Organisation: The ICD-10 Classification of Mental and Behavioural Disorders: Diagnostic Criteria for Research. Genf 1994

Panagiotakos DB, Pitsavos C, Chrysohoou C, Vlismas K, Skoumas Y, Palliou K, Stefanadis C (2008): Dietary habits mediate the relationship between socio-economic status and CVD factors among healthy adults: the ATTICA study. Public Health Nutr $\underline{11}$, 1342-1349

Pardee PE, Norman GJ, Lustig RH, Preud'homme D, Schwimmer JB (2007): Television viewing and hypertension in obese children. Am J Prev Med 33, 439-443

Pickering TG (2001): Mental stress as a causal factor in the development of hypertension and cardiovascular disease. Curr Hypertens Rep $\underline{3}$, 249-254

Rajmil L, Herdman M, Fernandez de Sanmamed MJ, Detmar S, Bruil J, Ravens-Sieberer U, Bullinger M, Simeoni MC, Auquier P (2004): Generic health-related quality of life instruments in children and adolescents: a qualitative analysis of content. J Adolesc Health $\underline{34}$, 37-45

Randich A, Maixner W (1986): The role of sinoaortic and cardiopulmonary baroreceptor reflex arcs in nociception and stress-induced analgesia. Ann N Y Acad Sci 467, 385-401 
Raskeliene V, Babarskiene MR, Macijauskiene J, Seskevicius A (2009): Impact of duration and treatment of arterial hypertension on health-related quality of life. Medicina (Kaunas) $\underline{45}, 405-411$

Rau H, Elbert T (2001): Psychophysiology of arterial baroreceptors and the etiology of hypertension. Biol Psychol $\underline{\text { 57, }}$ 179-201

Ravens-Sieberer U (2000): Verfahren zur Erfassung der gesundheitsbezogenen Lebensqualität bei Kindern und Jugendlichen. Bundesgesundheitsblatt Gesundheitsforschung Gesundheitsschutz $\underline{43}$, 198-209

Ravens-Sieberer U, Bullinger M (1998): Assessing health-related quality of life in chronically ill children with the German KINDL: first psychometric and content analytical results. Quality Life Res 7, 399-407

Ravens-Sieberer U, Hölling H, Bettge S, Wietzker A (2002): Erfassung von psychischer Gesundheit und Lebensqualität im Kinder- und Jugendgesundheitssurvey. Gesundheitswesen $\underline{64}$ (Sonderheft 1), 30-35

Ravens-Sieberer U, Ellert U, Erhart M (2007 a): Gesundheitsbezogene Lebensqualität von Kindern und Jugendlichen in Deutschland. Bundesgesundheitsblatt Gesundheitsforschung Gesundheitsschutz $\underline{50}, 810-818$

Ravens-Sieberer U, Wille N, Bettge S, Erhart M (2007 b): Psychische Gesundheit von Kindern und Jugendlichen in Deutschland. Bundesgesundheitsblatt Gesundheitsforschung Gesundheitsschutz $\underline{50}, 871-878$

Robbins MA, Elias MF, Croog SH, Colton T (1994): Unmedicated blood pressure levels and quality of life in elderly hypertensive women. Psychosom Med $\underline{56}$, 251-259

Rogeness GA, Cepeda C, Macedo CA, Fischer C, Harris WR (1990): Differences in heart rate and blood pressure in children with conduct disorder, major depression, and separation anxiety. Psychiatry Res 33, 199-206

Rostrup M, Kjeldsen SE, Eide IK (1990): Awareness of hypertension increases blood pressure and sympathetic responses to cold pressor test. Am J Hypertens $\underline{3}$, 912-917

Rostrup M, Mundal HH, Westheim A, Eide I (1991): Awareness of high blood pressure increases arterial plasma catecholamines, platelet noradrenaline and adrenergic responses to mental stress. J Hypertens $\underline{9}$, 159-166

Rush AJ, Linden M, Zobel A (2002): Vagus nerve stimulation. A potential therapy for chronic/recurrent depression? Fortschr Neurol Psychiatr $\underline{70}$, 297-302 
Saboya PM, Zimmermann PR, Bodanese LC (2010): Association between anxiety or depressive symptoms and arterial hypertension, and their impact on the quality of life. Int J Psychiatry Med $\underline{40}, 307-320$

Sackeim HA, Rush AJ, George MS, Marangell LB, Husain MM, Nahas Z, Johnson CR, Seidman S, Giller C, Haines S, et al. (2001): Vagus nerve stimulation (VNS) for treatment-resistant depression: efficacy, side effects, and predictors of outcome. Neuropsychopharmacology $\underline{25}, 713-728$

Scheidt-Nave C, Ellert U, Thyen U, Schlaud M (2007): Prävalenz und Charakteristika von Kindern und Jugendlichen mit speziellem Versorgungsbedarf im Kinder- und Jugendgesundheitssurvey (KiGGS) in Deutschland. Bundesgesundheitsblatt Gesundheitsforschung Gesundheitsschutz 50 , 750-756

Schneewind K, Beckmann M, Hecht-Jackl A: Familienklima-Skalen. Bericht. Institut für Psychologie - Persönlichkeitspsychologie und Psychodiagnostik der Ludwig Maximilians Universität, München 1985

Schumacher J, Klaiberg A, Brähler E: Diagnostische Verfahren zu Lebensqualität und Wohlbefinden. Hogrefe, Göttingen 2003

Schwandt P, Bertsch T, Haas GM (2010): Anthropometric screening for silent cardiovascular risk factors in adolescents: The PEP Family Heart Study. Atherosclerosis 211, 667-671

Schwarzer R, Jerusalem M: Skalen zur Erfassung von Lehrer- und Schülermerkmalen. Dokumentation der psychometrischen Verfahren im Rahmen der Wissenschaftlichen Begleitung des Modellversuchs Selbstwirksame Schulen. Freie Universität Berlin, Berlin 1999

Shafique S, Dalsing MC (2006): Vagus nerve stimulation therapy for treatment of drug-resistant epilepsy and depression. Perspect Vasc Surg Endovasc Ther 18, 323-327

Shapiro AP, Alderman MH, Clarkson TB, Furberg CD, Jesse MJ, Julius S, Miller RE, Pitt B (1987): Behavioral consequences of hypertension and antihypertensive therapy. Circulation $76,101-103$

Shi Y, de Groh M, Morrison H (2012): Increasing blood pressure and its associated factors in Canadian children and adolescents from Canadian Health Measures Survey. BMC Public Health $\underline{12}$, 388

Simonetti GD, Schwertz R, Klett M, Hoffmann GF, Schaefer F, Wuhl E (2011): Determinants of blood pressure in preschool children: the role of parental smoking. Circulation $\underline{123}, 292-298$

Sinha MD, Reid CJ (2007): Evaluation of blood pressure in children. Curr Opin Nephrol Hypertens $\underline{16}, 577-584$ 
Sommers-Flanagan J, Greenberg RP (1989): Psychosocial variables and hypertension: a new look at an old controversy. J Nerv Ment Dis 177, 15-24

Sorof JM, Alexandrov AV, Cardwell G, Portman RJ (2003): Carotid artery intimal-medial thickness and left ventricular hypertrophy in children with elevated blood pressure. Pediatrics $\underline{111}, 61-66$

Sorof JM, Lai D, Turner J, Poffenbarger T, Portman RJ (2004): Overweight, ethnicity, and the prevalence of hypertension in school-aged children. Pediatrics $\underline{113}, 475-482$

Spruill TM (2010): Chronic psychosocial stress and hypertension. Curr Hypertens Rep $\underline{12}, 10-16$

Stein JD, Brown GC, Brown MM, Sharma S, Hollands H, Stein HD (2002): The quality of life of patients with hypertension. J Clin Hypertens (Greenwich) 4 , 181-188

Sun SS, Grave GD, Siervogel RM, Pickoff AA, Arslanian SS, Daniels SR (2007): Systolic blood pressure in childhood predicts hypertension and metabolic syndrome later in life. Pediatrics $\underline{119}$, 237-246

Theorell T (1990): Family history of hypertension--an individual trait interacting with spontaneously occurring job stressors. Scand J Work Environ Health 16 Suppl 1, 74-79

Torrance B, McGuire KA, Lewanczuk R, McGavock J (2007): Overweight, physical activity and high blood pressure in children: a review of the literature. Vasc Health Risk Manag $\underline{3}, 139-149$

Trevisol DJ, Moreira LB, Fuchs FD, Fuchs SC (2012): Health-related quality of life is worse in individuals with hypertension under drug treatment: results of population-based study. J Hum Hypertens $\underline{26}$, 374-380

Tsioufis C, Kyvelou S, Tsiachris D, Tolis P, Hararis G, Koufakis N, Psaltopoulou T, Panagiotakos D, Kokkinos P, Stefanadis C (2011): Relation between physical activity and blood pressure levels in young Greek adolescents: the Leontio Lyceum Study. Eur J Public Health 21, 63-68

Tu W, Eckert GJ, DiMeglio LA, Yu Z, Jung J, Pratt JH (2011): Intensified effect of adiposity on blood pressure in overweight and obese children. Hypertension $\underline{58}, 818-824$

Winkleby MA, Ragland DR, Syme SL (1988): Self-reported stressors and hypertension: evidence of an inverse association. Am J Epidemiol 127, 124-134

Winkler J, Solzenberg H (1999): Der Sozialschichtindex im Bundes-Gesundheitssurvey. Gesundheitswesen 61 (Sonderheft 2), 178-183

Wong SN, Tz Sung RY, Leung LC (2006): Validation of three oscillometric blood pressure devices against auscultatory mercury sphygmomanometer in children. Blood Press Monit 11, 281-291 
Wustmann K, Kucera JP, Scheffers I, Mohaupt M, Kroon AA, de Leeuw PW, Schmidli J, Allemann Y, Delacretaz E (2009): Effects of chronic baroreceptor stimulation on the autonomic cardiovascular regulation in patients with drug-resistant arterial hypertension. Hypertension $\underline{54}$, 530-536

Youssef RM, Moubarak, II, Kamel MI (2005): Factors affecting the quality of life of hypertensive patients. East Mediterr Health J 11, 109-118

Zhang YX, Wang SR (2011): Monitoring of blood pressure in overweight and obese children in Shandong, China. Ann Hum Biol 38, 603-607 


\section{Danksagung}

Mein besonderer Dank gilt Herrn Prof. Dr. med. Herrmann-Lingen für die Überlassung des Themas dieser Dissertation und die Möglichkeit, in seiner Abteilung wissenschaftlich zu arbeiten. Ich bedanke mich für seine konstruktive Kritik und sein Interesse an meiner Arbeit.

Danken möchte ich Herrn Dr. med Hulpke-Wette dafür, dass er die Idee für mein Promotionsthema hatte und meine Arbeit initiiert hat.

Ein großer Dank gilt Herrn Prof. Dr. mult. Thomas Meyer für die sehr gute Zusammenarbeit bei der Publikation im Rahmen meiner Dissertation.

Überdies möchte ich mich auch ganz besonders bei der medizinischen Statistik der Universitätsmedizin Göttingen, insbesondere bei Simon Schneider, für die fachkundige und sehr hilfreiche Beratung bedanken. 


\section{Lebenslauf}

Am 09. April 1986 wurde ich, Angela Stefanie Berendes, als erste Tochter der Eheleute Karl-Heinz Berendes und Gudrun Berendes in Bamberg geboren.

Zusammen mit meiner jüngeren Schwester Carola wuchs ich in Braunschweig auf. Ab dem sechsten Lebensjahr besuchte ich die Grundschule in BraunschweigStöckheim, wechselte 1996 auf die Orientierungsstufe in BraunschweigStöckheim, bevor ich ab 1998 das Gymnasium Raabeschule, ebenfalls in Braunschweig, besuchte.

2005 erlangte ich dort die Allgemeine Hochschulreife und begann zum Wintersemester 2005 mit dem Medizinstudium an der Georg-August-Universität in Göttingen. Nach vier Semestern absolvierte ich den Ersten Abschnitt der Ärztlichen Prüfung. Ende 2009 begann ich meine Dissertation mit dem Titel „Beziehungen erhöhten arteriellen Blutdrucks zu Lebensqualität, Problembelastung und Risikoverhalten im Jugendalter" in der Abteilung Psychosomatische Medizin und Psychotherapie der Universitätsmedizin Göttingen unter der Leitung von Prof. Dr. med. Christoph Herrmann-Lingen. Im Sommer 2012 absolvierte ich erfolgreich den Zweiten Abschnitt der Ärztlichen Prüfung und stellte im Anschluss daran meine Dissertation fertig.

Ab Dezember 2012 begann ich als Assistenzärztin in der Kinder- und Jugendpsychiatrie in Bonn zu arbeiten. 